\title{
Results of Toxicity Tests and Chemical Analyses Conducted on Sediments Collected from the TNX Outfall Delta Operable Unit, July 1999
}

by

W. L. Specht

Westinghouse Savannah River Company

Savannah River Site

Aiken, South Carolina 29808
RECEIVED

FFR 232000

OSTI

DOE Contract No. DE-AC09-96SR18500

This paper was prepared in connection with work done under the above contract number with the U.S. Department of Energy. By acceptance of this paper, the publisher and/or recipient acknowledges the U.S. Government's right to retain a nonexclusive, royalty-free license in and to any copyright covering this paper, along with the right to reproduce and to authorize others to reproduce all or part of the copyrighted paper. 


\section{DISCLAIMER}

This report was prepared as an account of work sponsored by an agency of the United States Government. Neither the United States Government nor any agency thereof, nor any of their employees, makes any warranty, express or implied, or assumes any legal liability or responsibility for the accuracy, completeness, or usefulness of any information, apparatus, product or process disclosed, or represents that its use would not infringe privately owned rights. Reference herein to any specific commercial product, process or service by trade name, trademark, manufacturer, or otherwise does not necessarily constitute or imply its endorsement, recommendation, or favoring by the United States Government or any agency thereof. The views and opinions of authors expressed herein do not necessarily state or reflect those of the United States Government or any agency thereof.

This report has been reproduced directly from the best available copy.

Available for sale to the public, in paper, from: U.S. Department of Commerce, National Technical Information Service, 5285 Port Royal Road, Springfield, VA 22161

phone: (800) 553-6847

fax: (703) 605-6900

email: orders@ntis.fedworld.gov

online ordering: http://www.ntis.gov/ordering.htm

Available electronically at http://www.doe.gov/bridge

Available for a processing fee to U.S. Department of Energy and its contractors, in paper, from: U.S. Department of Energy, Office of Scientific and Technical Information, P.O. Box 62, Oak Ridge, TN 37831-0062

phone: (865)576-8401

fax: (865)576-5728

email: reports@adonis.osti.gov 


\section{DISCLAIMER}

\section{Portions of this document may be illegible in electronic image products. Images are produced from the best available original document.}




\title{
RESULTS OF TOXICITY TESTS AND CHEMICAL ANALYSES CONDUCTED ON SEDIMENTS COLLECTED FROM THE TNX OUTFALL DELTA OPERABLE UNIT, JULY 1999
}

\author{
by \\ WINONA L. SPECHT \\ SAVANNAH RIVER TECHNOLOGY CENTER
}

Approved by:

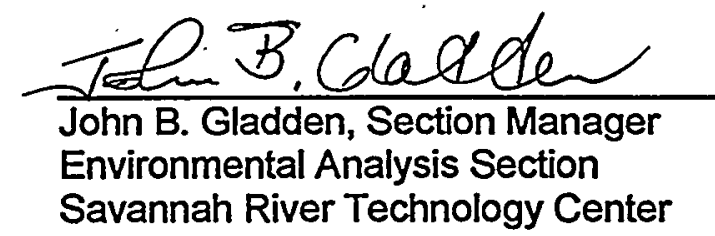

Publication Date: $\quad$ December 1999

WESTINGHOUSE SAVANNAH RIVER COMPANY SAVANNAH RIVER SITE AIKEN, SC 29808

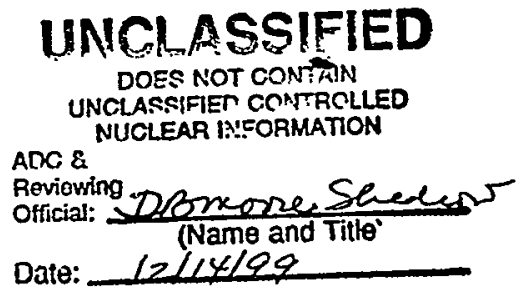

THIS DOCUMENT WAS PREPARED IN CONNECTION WITH WORK UNDER U.S. DEPARTMENT OF ENERGY CONTRACT DE-AC09-88SR18035. By acceptance of this paper, the publisher and/or recipient acknowledges the U.S. Government's right to retain a nonexclusive, royalty-free license in and to any copyright covering this paper, along with the right to reproduce and to authorize others to reproduce all or any part of the copyrighted paper. 


\section{TABLE OF CONTENTS}

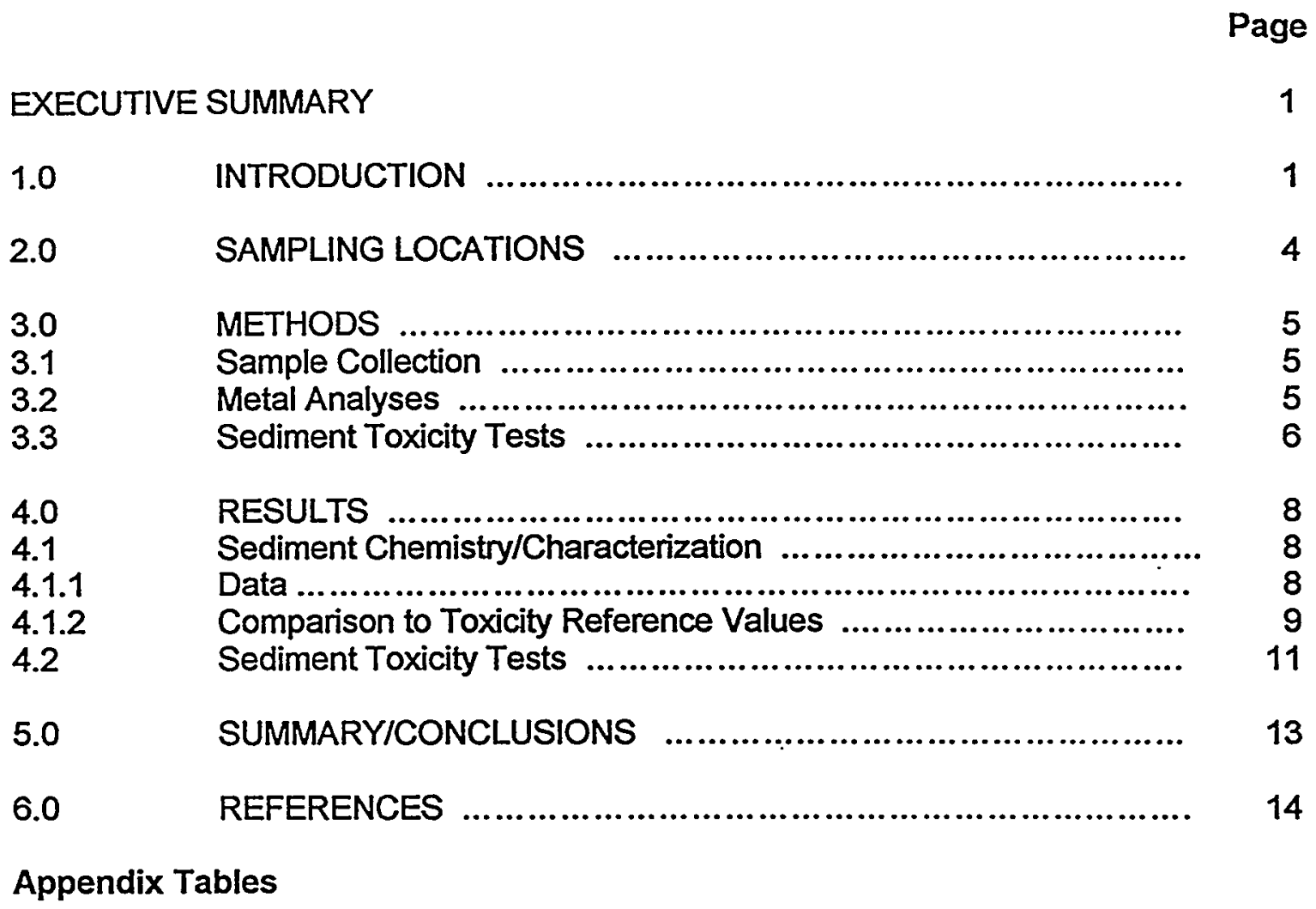

Appendix $1 \quad$ Sediment Toxicity Test Reports 


\section{List of Tables}

Page

Table 1 Sampling Locations for Metal Analyses and Toxicity Testing .......

Table 2 Analytical Methods and Minimum Detection Limits for Sediment Samples

Table 3 Test Conditions for Conducting a 10-day Sediment Toxicity Test with Hyalella azteca, following U.S. EPA Testing Protocol

Table 4 Test Conditions for Conducting a 10-day Sediment Toxicity Test with Chironomus tentans, following U.S. EPA Testing Protocol

Table 5 Results of Metal Analyses Conducted on Sediments Collected from TNX Inner Swamp, Phase I and/or II, and 1999 ...............

Table 6 Particle Size Distribution of TNXOD OU Sediments Used for Toxicity Testing

Table 7 Total Organic Carbon Concentrations Distribution of TNXOD OU Sediments Used for Toxicity Testing

Table 8 Results of TNX Inner Swamp Hyalella azteca Sediment Toxicity Tests and Unit Specific Background Toxicity Tests

Table 9 Percent Survival in Definitive Hyalella azteca Toxicity Tests at Locations OFD-A5 and OFD-D7

Table 10, Results of Chironomus tentans Toxicity Tests

\section{List of Figures}

Figure 1 Location of TNX ODU

Figure 2 TNXOD OU / 1999 Sampling Locations

Figure 3 Mercury Concentrations in TNX Sediments

$\begin{array}{ll}\text { Figure } 4 & \text { Uranium Concentrations in TNX Sediments } \\ \text { Figure } 5 & \text { Percent Mortality in Hyalella azteca Toxicity Tests }\end{array}$ 


\section{EXECUTIVE SUMMARY}

In order to provide unit specific toxicity data that will be used to address critical uncertainty in the ecological risk assessment (ERA) for the TNX Outfall Delta Operable Unit (TNXOD OU), sediments were collected from eight locations in the Inner Swamp portion of the operable unit and two unit specific background locations. These samples were analyzed for total mercury, total uranium, and sediment toxicity.

The results of the chemical analyses indicate that the locations that were sampled contained elevated levels of mercury and/or uranium, as compared to unit specific background locations. Background concentrations of mercury were $\leq 0.12 \mathrm{mg} / \mathrm{kg}$, while concentrations in the Inner Swamp ranged from 0.2 to $18.4 \mathrm{mg} / \mathrm{kg}$. Background concentrations of uranium were $<5 \mathrm{mg} / \mathrm{kg}$, while concentrations in the Inner Swamp ranged from 62.6 to $254 \mathrm{mg} / \mathrm{kg}$.

The results of toxicity tests conducted on sediments collected from the Inner Swamp and the background locations indicated that 9 of the 10 locations (including both of the background locations) were toxic to Hyalella azteca. The toxicity was not related to contaminant concentrations and does not appear to be due to soil type (high clay) and/or lack of organic matter in the sediment material. Additional tests were performed using a different species (Chironomus tentans) on sediment collected from the location expected to have the highest level of mercury and one of the background locations. The results of these tests also indicated that both sediments were toxic to the test organisms. However, the sediment sample that contained very high levels of mercury and uranium were less toxic than the background reference location, which suggests that the observed toxicity to benthic dwelling invertebrates is not directly attributable to concentrations of these constituents in sediments. Dilution series toxicity tests were also performed on sediment collected from two locations, but it was not possible to determine an LC50 (lethal concentration, 50\%; the concentration that results in mortality to $50 \%$ of the test organisms) for mercury or uranium since toxicity was unaffected by dilution.

In summary, the results of these three versions of sediment toxicity tests at the TNXOD OU indicate that the toxicity observed in the Inner Swamp sediments is not related to mercury or uranium concentrations.

\subsection{INTRODUCTION}

The TNX area of the Savannah River Site is adjacent to the Savannah River flood plain at River Mile 156 (Figures 1 and 2). TNX served as a pilot-scale testing and evaluation facility in support of chemical process for fuel and target manufacturing areas and the Defense Waste Processing Facility. Prior to 1988, wastewater generated as result of these activities was discharged to unlined earthen basins through a network of process sewers. The old TNX seepage basin was an unlined earthen excavation designed to remove wastewater solids (in the inlet section) and contain wastewater (in the seepage section) until it could infiltrate into the underlying sediments that were believed to act as a natural ion exchange media. The basin was operated from 1958 to 1980 . In the spring of 1980 , the wastewater flow to the basin was stopped. As part of the basin closure, the west wall of the basin was breached to drain the standing free liquids into the adjacent wetlands. The breaching, along with the basin overflow throughout its 22-year operating history, resulted in a discharge gully and outfall delta in the adjacent swamp. Among 


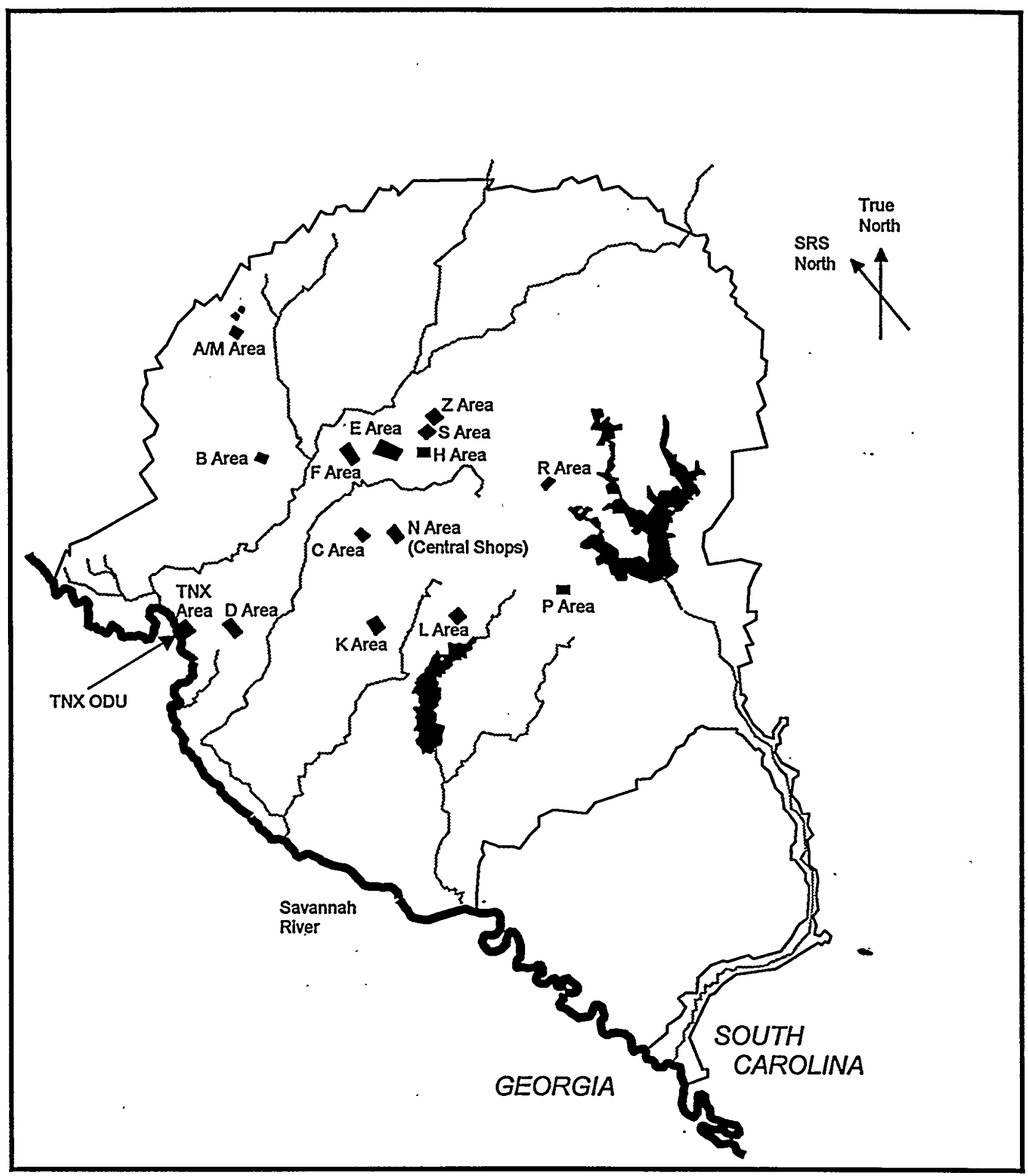

Figure 1. Location of TNX ODU 


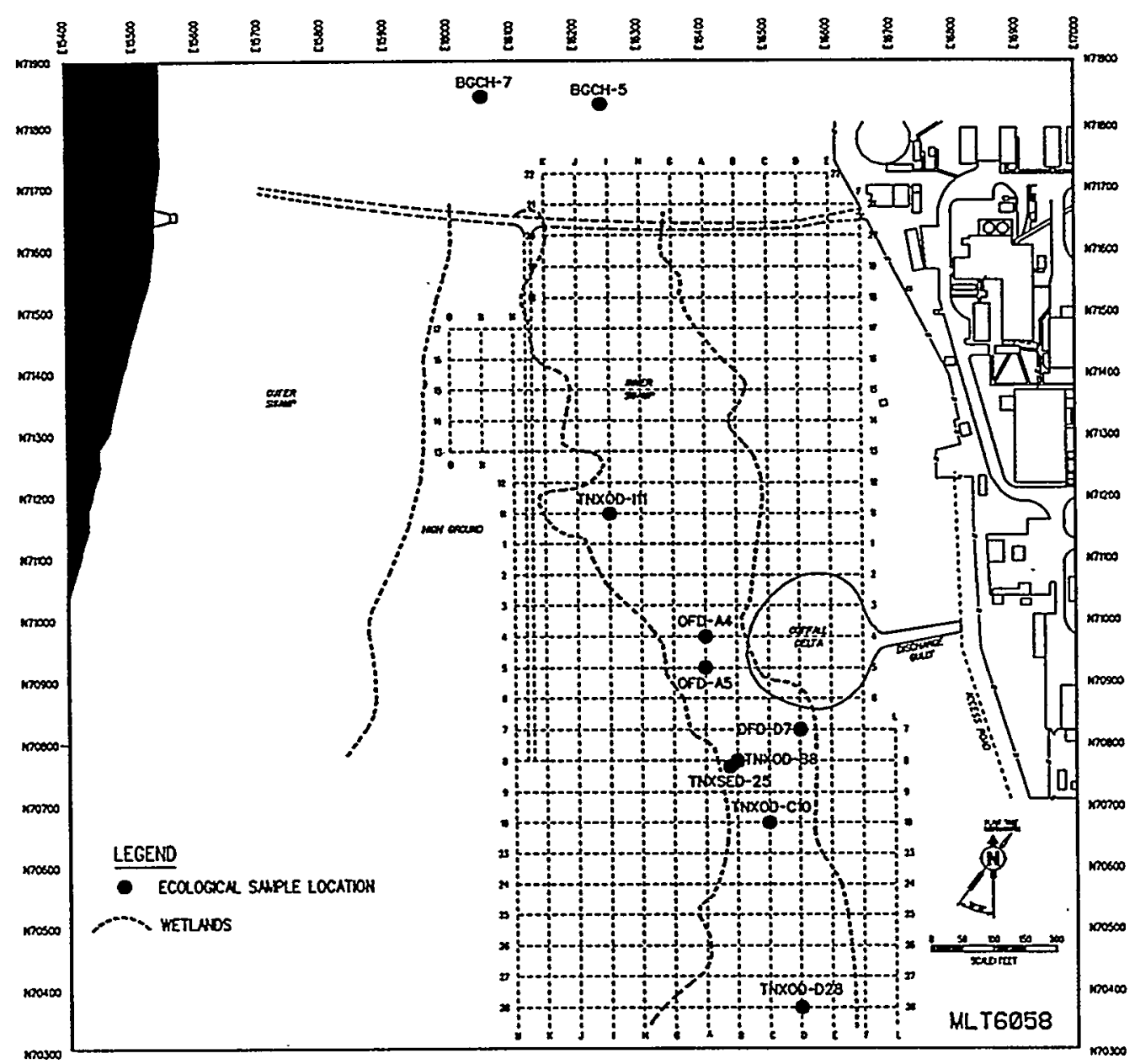

Figure 2. TNXOD OU / 1999 Sampling Locations

the significant wastes discharged to the basin (and subsequently to the swamp) were mercury and depleted uranium.

Stormwater continues to be discharged from the TNX area via a concrete culvert located in the discharge gully. This stormwater discharge deposits "clean" sand and debris onto the surface of the outfall delta and flows into the Inner Swamp (Figure 2). The Inner Swamp in turn drains into and/or mixes with water from the nearby Savannah River.

In the spring of 1998, the portion of the TNX Outfall Delta Operable Unit (TNXOD OU) was separated from the TNX Area Operable Unit. The TNXOD OU consists of the lower portion of the Discharge Gully, the Outfall Delta, Inner Swamp, High Ground, and Outer Swamp exposure groups. 
Analytical data from both the Phase I (1995-1996) and Phase II (1998) Recovery Act Facility Investigation/Remedial Investigation (RFI/RI) sampling activities are currently being evaluated. Preliminary results of the ecological risk assessment (ERA) for the TNX Inner Swamp sediment exposure group suggest that mercury is the significant constituent of potential concem (COPC) with regard to the potential for toxic effects to sediment dwelling invertebrate communities in this wetlands area. Mercury was detected in 97 of 98 samples, with six of the detections being estimated values. The range of detections was wide $(0.007 \mathrm{mg} / \mathrm{kg}$ to $30.8 \mathrm{mg} / \mathrm{kg})$ compared to the unit specific background concentrations $(0.007 \mathrm{mg} / \mathrm{kg}$ to $0.12 \mathrm{mg} / \mathrm{kg}$ ). Mercury is known to be related to prior TNX processes; its distribution in the Inner Swamp indicates that it is unit related. Some areas of the Inner Swamp may also contain elevated concentrations of uranium. Although the activity levels reported for U-233/234, U-235 and U-238 (Phase I and II) are not high enough to be of ecological concern from a radiological standpoint, uranium may be present at concentrations that could produce a toxic response as a heavy metal. Therefore, even though uranium was not identified as a final COPC in the ERA, it was included as an analyte in this investigation.

In July 1999 sediment samples were collected from eight locations in the Inner Swamp and two unit specific reference locations. The samples were analyzed for total mercury and total uranium and sediment toxicity tests were performed to determine if there is an accumulation of these metals at toxic levels in the inner Swamp wetlands area. This report summarizes the results of these tests. The results will be used to address critical uncertainty in the ecological risk assessment, particularly regarding the literature-based sediment toxicity reference value for mercury. The objective of the toxicity testing effort is to estimate an LC50 (lethal concentration, 50\%; the concentration that results in mortality to $50 \%$ of the test organisms). It is assumed that there is a true threshold, or concentration below which there is no adverse effect on sediment dwelling biota, and above which there is an adverse effect. Interpretation of the results of the toxicity tests and chemical analyses should identify where that threshold lies.

\subsection{SAMPLING LOCATIONS}

Based on the Phase I and II results of mercury analyses and uranium activity levels conducted on sediments collected from locations in the TNXOD Inner Swamp, eight locations were selected for additional analyses (Table 1, Figure 2). Samples were selected that were expected to have elevated mercury levels, elevated uranium levels, and elevated levels of both mercury and uranium. Thus the locations selected should represent worst case conditions in the TNXOD Inner Swamp. Two uncontaminated reference locations were also selected. These locations are within the Savannah River floodplain, but are located approximately $50 \mathrm{~m}$ upriver from the TNXOD Inner Swamp and were not known to be exposed to contaminants from TNX discharges.

All of the sampling locations lie within the floodplain of the Savannah River and contain hydric soils. Although most of the locations contained some standing water at the time of sampling, two of the locations were not inundated. For consistency, and because mercury is more of a concem in aquatic ecosystems than in terrestrial ecosystems, it was conservatively decided that sediment toxicity tests would be performed on all of the samples, rather than performing a terrestrial toxicity test on soil from the drier locations. A wet and a dry unit specific reference location were chosen so that data from the inner Swamp locations could be compared to a unit specific reference location that had similar hydrology. Based on existing data, sites were selected which had very low to very high 
levels of mercury and also very low to high levels of uranium (Table 1). If toxicity is detected and is due to either metal or a combination of the two metals, this selection of sampling locations should allow for a determination of which metal or combination of metals was responsible for the observed toxicity.

Table 1. Sampling Locations for Metal Analyses and Toxicity Testing

\begin{tabular}{lcll} 
Location & Wet/Dry & Toxicity Tests & Rationale for Selection \\
\hline TNXOD Inner & & & \\
\hline Swamp & & & \\
OFD-A4 & wet & Hyalella P/F test & high Hg; low U \\
TNXOD-B8 & wet & Hyalella P/F test & moderate Hg; high U \\
TNXOD-C10 & wet & Hyalella P/F test & low Hg; high U \\
TNXOD-D28 & wet & Hyalella P/F test & very low Hg; high U \\
TNXOD-111. & dry & Hyalella P/F test & very low Hg; low U \\
TNXSED-25 & wet & Hyalella P/F test & low Hg; high U \\
OFD-A5 & wet & Hyalella dilution series & high Hg; high U \\
OFD-D7 & dry & Hyalella dilution series & very high Hg; very low U \\
& & Chironomus P/F test &
\end{tabular}

\section{Unit Specific \\ Reference \\ BGCH-5 dry Hyalella P/F test unit specific background

BGCH-7 wet Hyalella P/F test unit specific background \\ 'Based on chemical data collected as part of the Phase I and II sampling investigations}

\subsection{METHODS}

\subsection{Sample Collection}

Sediment samples were collected in accordance with the protocols identified in the EPA Region IV Standard Operating Procedures Quality Assurance Manual (EPA 1996), WSRC Hydrogeologic Data Collection Procedures (U), Manual 3Q5 (WSRC 1997), and Standard Guide for Collection, Storage, Characterization, and Manipulation of Sediments for Toxicological Testing (ASTM 1991). Savannah River Technical Center personnel observed the collection of the samples.

Sediment samples were collected with a hand auger that sampled the top 12 inches (30 $\mathrm{cm}$ ) of sediment. The sediment collected was placed on a clean sheet of polyethylene and mixed thoroughly with a stainless steel spoon. A subsample was then placed in a plastic bottle for chemical analyses and the remainder of the sample was placed in a plastic jar for toxicity testing. The samples were placed in a cooler, iced, and transported to certified laboratories for analysis.

\subsection{Metal Analyses}

Definitive level total mercury and total uranium analyses of the sediment samples were conducted by a certified laboratory (General Engineering Laboratory, Charleston, SC; SCDHEC Certification \# 10120) using the U.S. EPA SW-846 methods specified in Table 2. In interpreting the results, if a result was less than the detection limit, $50 \%$ of the sample specific method detection limit (ssmdl) was reported. 
Table 2. Analytical Methods and Minimum Detection Limits for Sediment Samples

\begin{tabular}{|c|c|c|c|}
\hline Analyte & Digestion Method & Analytical Method & SSMDL* \\
\hline Mercury & Aqua Regia & EPA7471A & 3.2 to $94.3 \mu \mathrm{g} / \mathrm{kg}$ \\
\hline Uranium & EPA3050B & EPA6020 & 6.5 to $18.3 \mu \mathrm{g} / \mathrm{kg}$ \\
\hline
\end{tabular}

\subsection{Sediment Toxicity Tests}

Typical 10-day amphipod sediment toxicity tests (EPA 1994) are set as pass/fail (P/F) tests with a control treatment and sample treatment. However, the objective of this toxicity testing effort is to estimate an LC50, which is not calculable in individual Pass/Fail tests. It is assumed that there is a true threshold, or concentration below which there is no adverse effect on sediment dwelling biota, and above which there is an adverse effect. The American Society for Testing and Materials (ASTM 1994) describes a dilution series toxicity test that would allow for the calculation of a threshold .. concentration. This testing scheme provides a gradient of concentrations to allow for a comprehensive interpretation of all of the test results in an effort to estimate as closely as possible where that threshold lies. Based on data collected in the Phase I characterization effort, a location expected to have high concentrations of mercury and uranium (OFD-A5) and a location expected to have a high concentration of mercury and low concentration of uranium (OFD-D7) were selected for dilution series tests, the rationale being that the LC50 values derived from these tests would be able to differentiate between toxicity due to mercury and toxicity due to a combination of the two metals.

10-day survival toxicity tests were conducted on the sediment samples from the ten locations using the amphipod, Hyalella azteca as the test species. Toxicity tests were also performed on the TNX sediment expected to have the highest mercury concentration (OFD-D7; based on Phase I data) and on the corresponding reference sediment (BGCH-5) using a different species (Chironomus tentans). All tests were performed by a laboratory certified to perform biological testing (ETT Environmental, Greenville, SC; SCDHEC Certification \#23104001); however, SCDHEC currently has no recommended protocols for sediment toxicity tests. The testing was performed following EPA Test Methods 100.1, Hyalella azteca 10-day Survival Test for Sediments and 100.2 Chironomus tentans 10-day Survival and Growth Test for Sediments (U.S. EPA, 1994): Test conditions for the Hyalella azteca and Chironomus tentans tests are summarized in Tables 3 and 4, respectively.

The reference sediment used for the toxicity tests was collected from Resurrection Creek, Greenville County, SC. ETT Environmental routinely uses sediment from this location as a control sediment and has demonstrated that it consistently meets the survival criterion for a control sediment. 
Table 3. Test Conditions for Conducting a 10-day Sediment Toxicity Test with Hyalella azteca, following U.S. EPA Testing Protocol

Parameter

Test type

Temperature

Light quality

Light intensity

Photoperiod

Test chamber

Sediment volume

Overtying water volume

Water renewal

Age of organisms

Number of organisms/chamber

Number of replicates/treatment

Feeding

Aeration

Overlying water

Overlying water quality parameters

Test duration

Endpoint

Test acceptability
Conditions

Whole sediment toxicity test with renewal of overlying water $23 \pm 1^{\circ} \mathrm{C}$

Wide-spectrum fiuorescent lights

500 to 1000 lux

16L:8D

$300 \mathrm{ml}$ beaker

$100 \mathrm{ml}$

$175 \mathrm{ml}$

2 volume additions/day

7 to 14 days at start of test

10

8

YCT food, $1.5 \mathrm{~m} /$ day/test chamber

None, unless DO drops below $40 \%$ of saturation

Surface water

Hardness, alkalinity, conductivity, $\mathrm{pH}$, and ammonia at beginning and end of test, temperature and DO daily 10 days

Survival

Control survival $\geq 80 \%$

Table 4. Test Conditions for Conducting a 10-day Sediment Toxicity Test with Chironomus tentans, following U.S. EPA Testing Protocol

Parameter

Test type

Temperature

Light quality

Light intensity

Photoperiod

Test chamber

Sediment volume

Overlying water volume

Water renewal

Age of organisms

Number of organisms/chamber

Number of replicates/treatment

Feeding

Aeration

Overlying water

Overlying water quality parameters

Test duration

Endpoint

Test acceptability
Conditions

Whole sediment toxicity test with renewal of overlying water $23 \pm 1^{\circ} \mathrm{C}$

Wide-spectrum fluorescent lights

500 to 1000 lux

16L:8D

High-form lipless $300 \mathrm{mi}$ beaker

$100 \mathrm{ml}$

$175 \mathrm{ml}$

2 volume additions/day

3rd instar larvae

10

8

Tetrafin goldfish food $1.5 \mathrm{ml}$ dailyftest chamber

None, unless DO drops below $40 \%$ of saturation

Surface water

Hardness, alkalinity, conductivity, $\mathrm{pH}$, and ammonia

at beginning and end of test; temperature and DO daily

10 days

Survival

Control survival $\geq 70 \%$ and mean weight per surviving control organism of $0.6 \mathrm{mg}$. 


\subsection{RESULTS}

\subsection{Sediment Chemistry/Characterization}

\subsubsection{Data}

The sediment chemistry data are summarized in Table 5 and Figures 3 and 4. Sediment characterization data (particle size distribution, organic matter content, and percent moisture) are presented in Tables 6 and 7.

Mercury concentrations at the two TNXOD background locations were 0.05 and 0.12 $\mathrm{mg} / \mathrm{kg}$, which are within the range of values for uncontaminated soils in the U.S., as reported by Kabata-Pendias and Pendias, 1992 (range of 0.02 to $1.50 \mathrm{mg} / \mathrm{kg}$; mean of $0.17 \mathrm{mg} / \mathrm{kg}$ ) and uncontaminated wetland soils on the SRS and in South Carolina, as reported by Dixon et al. (1997). Dixon et al. reported that mercury concentrations in uncontaminated wetland soils collected from 50 locations on the SRS ranged from $<0.055$ to $0.3 \mathrm{mg} / \mathrm{kg}$ and averaged $0.052 \mathrm{mg} / \mathrm{kg}$. At the 25 off-site locations that were sampled, mercury concentrations in wetland soils ranged from $<0.023$ to $0.74 \mathrm{mg} / \mathrm{kg}$ and averaged $0.043 \mathrm{mg} / \mathrm{kg}$. Three of the eight locations that were sampled in the TNXOD Inner Swamp had relatively low concentrations of mercury in the sediments $(<0.4$ $\mathrm{mg} / \mathrm{kg}$ ), while five of the locations had mercury concentrations $>2 \mathrm{mg} / \mathrm{kg}$ (Table 5; Figure 3). Location OFD-A4 had substantially more mercury than any of the other sampling locations (18.4 mg/kg).

Table 5. Results of Metal Analyses Conducted on Sediments Collected from TNXOD Inner Swamp, Phase I and/or II, and 1999

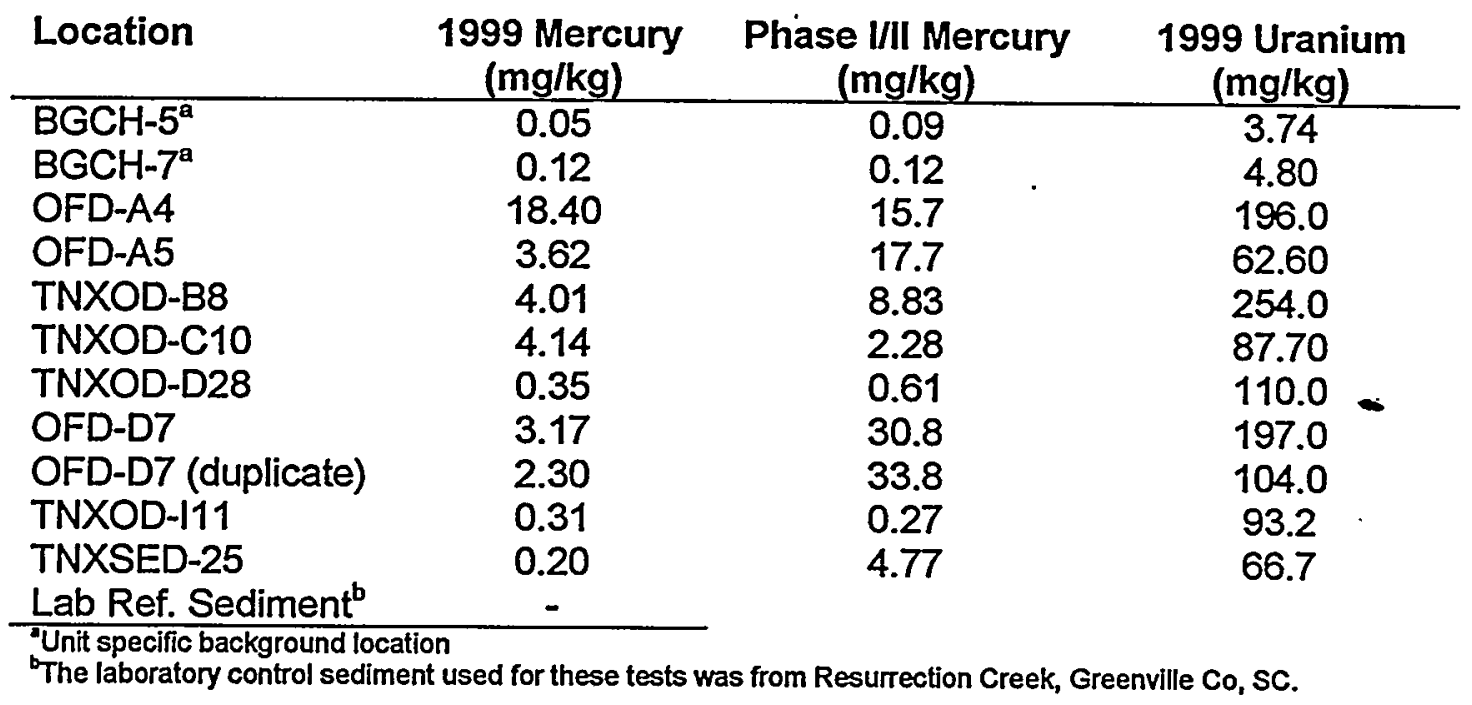

A comparison of mercury concentrations measured in Phase I and/or Phase II and 1999 at the same locations indicates that the concentrations measured were generally similar. Notable exceptions were OFD-A5 and OFD-D7, both of which had much lower concentrations of mercury in 1999 than in 1996 (Table 5). It should be noted that that the Phase I maximum result of $30.8 \mathrm{mg} / \mathrm{kg}$ from location OFD-D7 is flagged with a " $\mathrm{V}$ " analysis qualifier (WSRC 1996) indicating method blank contamination, potentially 
resulting in an over-estimation of the actual concentration. Overall, these results suggest that the distribution of mercury in the TNX Inner Swamp may be very patchy.

Uranium concentrations in the TNXOD OU unit specific reference sediments were $<5$ $\mathrm{mg} / \mathrm{kg}$, which are within the range of values for uncontaminated soils in the U.S., as reported by Kabata-Pendias and Pendias, 1992 (range of 0.3 to $10.7 \mathrm{mg} / \mathrm{kg}$; mean of 3.7 $\mathrm{mg} / \mathrm{kg}$ ). Uranium concentrations in the TNXOD Inner Swamp sediments ranged from 62.6 to $254 \mathrm{mg} / \mathrm{kg}$ (Table 5, Figure 4). Location TNXOD-B8 had the highest concentration of uranium, but locations OFD-A4 and OFD-D7 also had relatively high concentrations ( $200 \mathrm{mg} / \mathrm{kg})$.

The particle size distribution of the TNX sediments (Table 6) indicates that all of the sediments were composed primarily of sands $(0.06$ to $2 \mathrm{~mm})$, with lesser amounts of clay and silt $(<0.04 \mathrm{~mm})$ and gravel $(>2.0 \mathrm{~mm})$. Total organic carbon concentrations in all of the TNX sediments (Table 7) were very low $(<1 \mathrm{mg} / \mathrm{kg}$ ).

Table 6. Particle Size Distribution of TNXOD OU Sediments Used for Toxicity Testing

\begin{tabular}{|c|c|c|c|c|c|c|}
\hline \multirow[b]{2}{*}{ Location } & \multicolumn{6}{|c|}{ Particle Size Distribution by Percentage } \\
\hline & $<0.12 \mathrm{~mm}$ & $0.12-0.27 \mathrm{~mm}$ & $0.27-0.50 \mathrm{~mm}$ & $0.5-0.8 \mathrm{~mm}$ & $0.8-2.0 \mathrm{~mm}$ & $>2.0 \mathrm{~mm}$ \\
\hline $\mathrm{BGCH}-5^{2}$ & 14.4 & 24.3 & 28.8 & 16.4 & \begin{tabular}{ll|}
13.8 \\
\end{tabular} & 2.2 \\
\hline $\mathrm{BGCH}-7^{2}$ & 9.5 & 11.2 & 14.2 & 16.2 & 31.2 & 17.7 \\
\hline OFD-A4 & 11.8 & 12.3 & 11.7 & 13.0 & 30.0 & 21.2 \\
\hline OFD-A5 & 10.9 & 9.9 & 10.3 & 6.4 & 60.0 & 2.5 \\
\hline TNXOD-B8 & 20.0 & 19.8 & $24: 6$ & 15.9 & 15.4 & 4.3 \\
\hline TNXOD-C10 & 19.7 & 31.8 & 22.8 & 12.4 & 9.6 & 3.7 \\
\hline TNXOD-D28 & 16.2 & 18.4 & 28.3 & $17: 3$ & 14.8 & 4.9 \\
\hline OFD-D7 & 13.0 & 13.5 & 24.5 & 23.6 & 21.4 & 4.0 \\
\hline TNXOD-111 & 13.9 & 23.5 & 31.9 & 18.8 & 10.5 & 1.5 \\
\hline TNXSED-25 & 22.8 & 21.2 & 28.4 & 14.9 & 11.1 & 1.6 \\
\hline
\end{tabular}

Table 7. Total Organic Carbon Concentrations of TNXOD OU Sediments Used for Toxicity Testing

\begin{tabular}{lc} 
Location & Total Organic Carbon $(\mathrm{mg} / \mathrm{kg})$ \\
\hline BGCH-5 & 534 \\
BGCH-7 & 155 \\
OFD-A4 & 305 \\
OFD-A5 & 298 \\
TNXOD-B8 & 317 \\
TNXOD-C10 & 160 \\
TNXOD-D28 & 271 \\
OFD-D7 & 331 \\
TNXOD-111 & 193 \\
TNXSED-25 & 235 \\
\hline
\end{tabular}

\subsubsection{Comparison to Toxicity Reference Values}

The toxicity reference value (TRV) used for mercury in the ERA document is an Effects Range- Low (ER-L) value of $0.15 \mathrm{mg} / \mathrm{kg}$, as described in Toxicological Benchmarks for Screening Contaminants of Potential Concem for Effects on Sediment Associated Biota (Jones et al. 1997). The ER-L value may significantly over predict the likelihood of real effects. This is important because chemical concentrations may not be accurate. predictors of biological and ecological effects. This is because the percentage of the 


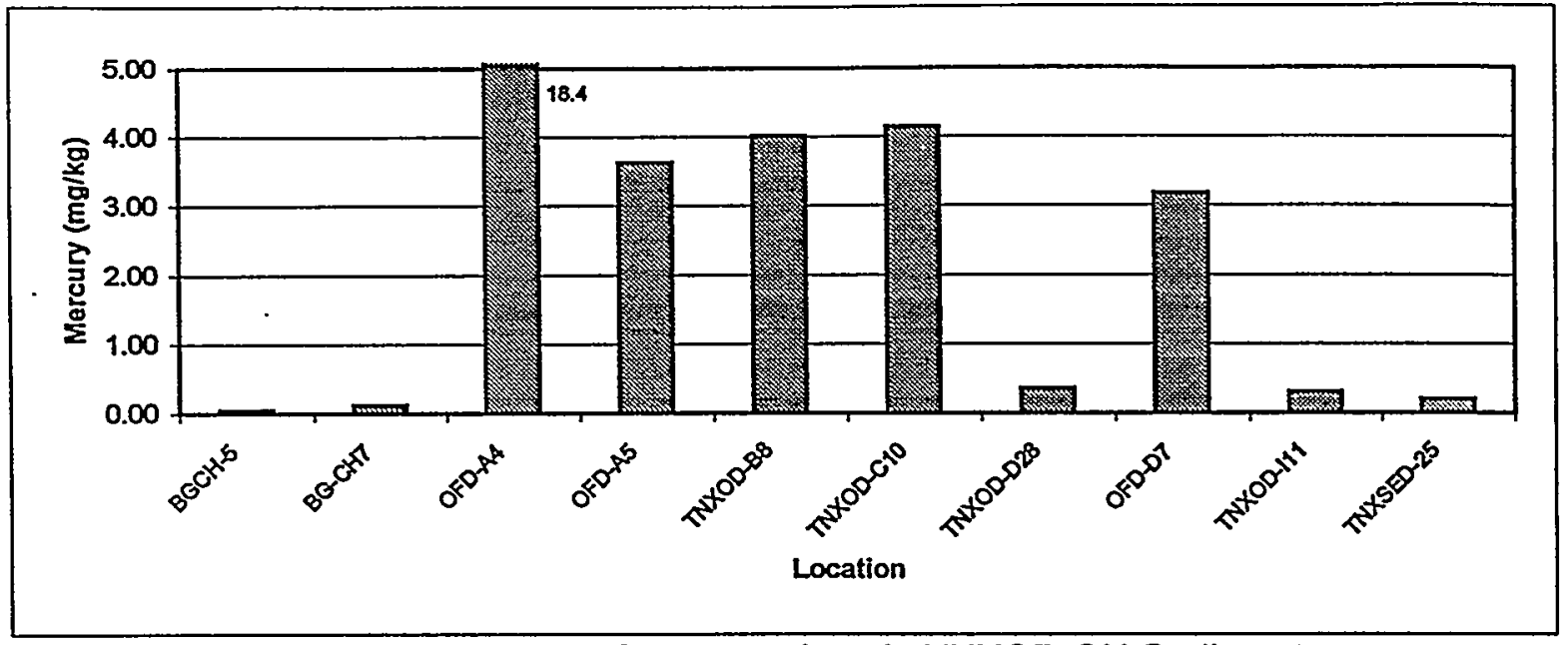

Figure 3. Mercury Concentrations in TNXOD OU Sediments

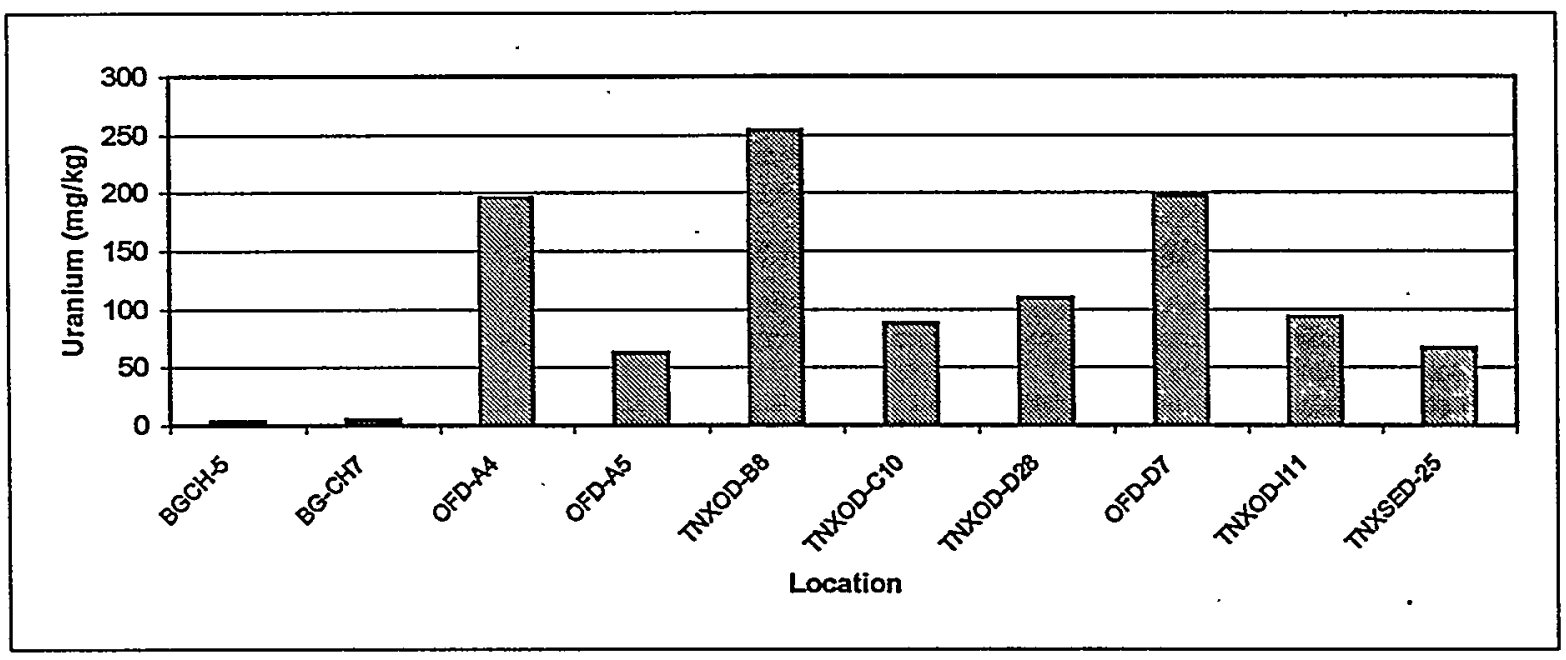

Figure 4. Uranium Concentrations in TNXOD OU Sediments

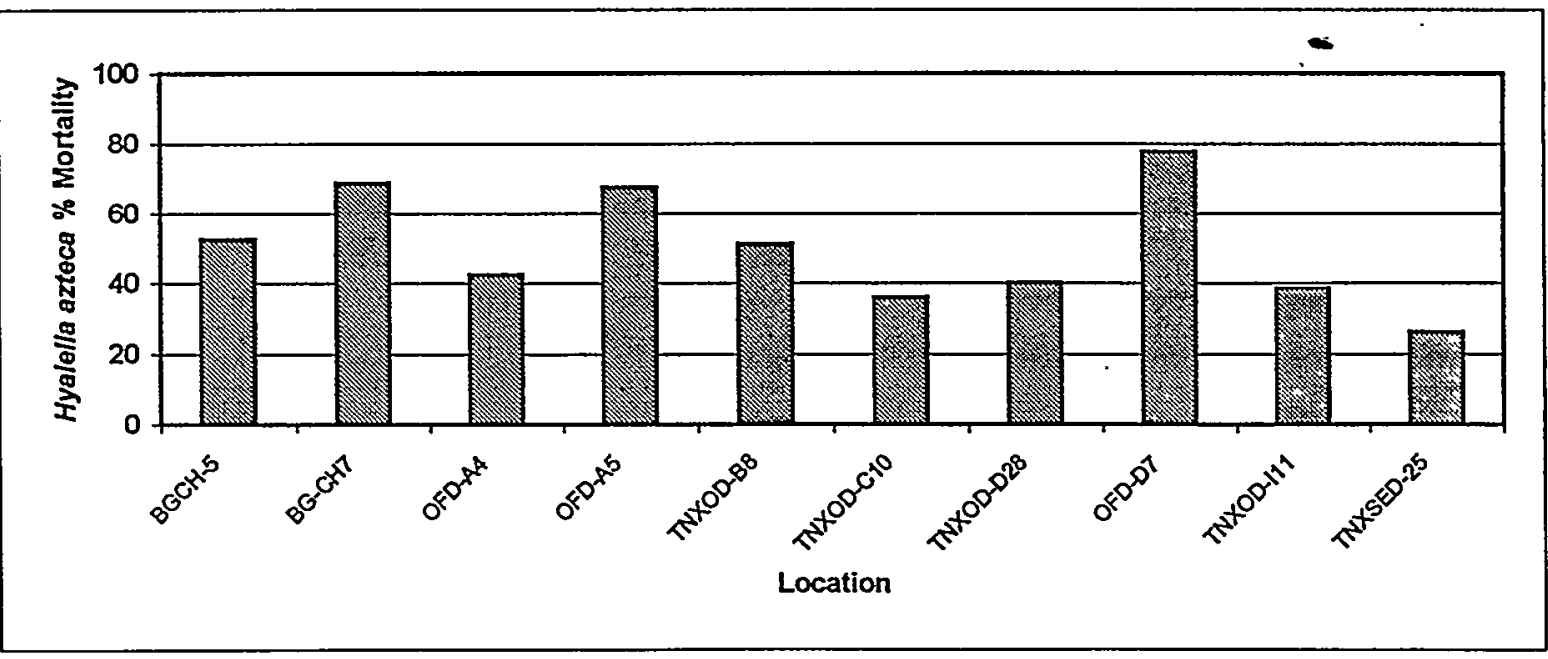

Figure 5. Percent Mortality in Hyalella azteca Toxicity Tests 
WSRC-TR-99-00380

chemical that is bioavailable may range from $0-100 \%$. Benchmarks may be used to determine which chemicals present in the sediment are most likely causing toxicity. The use of site specific biological data, such as toxicity tests, enables a more accurate evaluation of potential for adverse ecological impact. All of the eight locations sampled in the TNX Inner Swamp exceeded the TRV for mercury. A TRV for uranium is not available.

\subsection{Sediment Toxicity Tests}

The results of the sediment toxicity tests conducted on Hyalella azteca are summarized in Table 8 and 9 and Figure 5; detailed results are presented in Appendix 1. The results of the pass/fail tests indicate that, with the exception of TNXSED-25, all of the sediments, including both of the unit specific background locations (BGCH-5 and BGCH-7), had significant mortality as compared to the control sediment. Of the 10 locations that were tested, the unit specific background locations were among the most toxic locations tested, even though these locations had very low levels of mercury and uranium. Correlation coefficients were calculated for mercury concentrations vs. percent mortality and uranium concentrations vs. percent mortality. The results of the analyses indicate a correlation coefficient of -0.06385 for mercury vs. mortality and 0.031224 for uranium vs. mortality. These very low correlation coefficients (a value of 1.0 indicates perfect positive correlation, a value of -1.0 indicates perfect inverse correlation, and a value of 0.0 indicates no correlation) indicate that there is no relationship between the concentrations of mercury and uranium in the TNXOD sediments and Hyalella azteca mortality. As part of the RFI/RI investigation for TNXOD analyses for TAL, TCL, pesticides, PCB's and radionuclides were performed on the unit specific background sediments, as well as the operable unit sediments. In all, analyses for 161 chemical constituents and 45 radiological constituents were measured (WSRC 1999). The results indicated that none of the analytes were present at the unit-specific background locations in concentrations that would be expected to produce toxicity. Therefore, it is likely that other factors are responsible for the observed toxicity.

As discussed in Section 3.3, definitive (dilution series) Hyalella azteca toxicity tests were performed on the location that was expected to have the highest concentration of mercury and the location expected to have the highest concentration of uranium (based on sediment chemistry data collected in Phase I and/or II). These locations were OFD-D7 (high mercury) and OFD-A5 (high uranium). The definitive tests were conducted with the intent of being able to determine LC50's for mercury and ucanium. The results of the definitive tests showed no clear relationship between sediment concentration and toxicity (Table 9). For OFD-D7, the undiluted sediment was the most toxic concentration (22.5\% survival). However, when OFD-D7 sediment was diluted with the laboratory reference sediment to a concentration of $12.5 \%$, percent mortality was higher (55\%) than was a dilution of $25 \%$. For the sediment from OFD-A5, the most dilute test $(12.5 \%)$ had the highest mortality $(69 \%)$. There is no obvious explanation for these results. However, it was not possible to calculate LC50's for either mercury or uranium based on the results of the tests. Also, much lower levels of mercury were measured at both of these locations in 1999 than in Phase I and/or Phase II RFI/RI investigations.

Communication with the laboratory that performed the Hyalella azteca toxicity tests indicated that most of the sediments that were tested appeared to be clayey and had low organic matter. Hyalella azteca prefers sediments with more organic matter and less 
clay. It was suggested that toxicity tests be conducted using Chironomus tentans, which is somewhat more tolerant of heavy soils (personal communication, Dr. Robert Kelley, ETT Environmental). Based on existing chemical data, the location that was expected to have the highest concentration of mercury (OFD-D7) was chosen for further testing. For comparison, a $C$. tentans test was also performed on the unit specific background location that was dry (BGCH-5). The results of these tests indicate that percent mortality of $C$. tentans from both sediments was significantly higher than that of the laboratory reference sediment (Table 10). Percent mortality in the reference sediment was $33 \%$, as compared to $50 \%$ in OFD-D7 and $85 \%$ in BGCH-5. These results suggest that both sediments were unsuitable to $C$. tentans, but the very low recovery from BGCH-5 again suggests that the toxicity is not due to the presence of chemical contaminants, but is more likely due to unsuitable sediment characteristics. However, an examination of particle size distribution and percent organic matter in the sediments that were tested showed no clear relationship between either of these parameters and toxicity.

TNXSED25 had the highest percentage of fine particles $(<0.12 \mathrm{~mm})$, and yet was the only sediment that was not toxic. Concentrations of total organic carbon in the TNX sediments ranged from 155 to $534 \mathrm{mg} / \mathrm{kg}$, Organic carbon in the TNXSED 25 sediment was $235 \mathrm{mg} / \mathrm{kg}$. Therefore, there is no obvious relationship between the sediment characteristics that were measured and sediment toxicity. However, as previously discussed, toxicity was not related to mercury or uranium concentrations, and no other chemical constituents were identified in the Phase I and/or Phase II RFI/RI investigations that were present in potentially toxic concentrations in the TNXOD sediments.

Table 8. Results of TNXOD Inner Swamp Hyalella azteca Sediment Toxicity Tests and Unit Specific Background Toxicity Tests

\begin{tabular}{lcc} 
Location & Percent Mortality & Significant Mortality $(p=0.05)$ \\
\hline Reference Sediment & a & \\
TNXOD Inner Swamp & 16.0 & Yes \\
OFD-A4 & 42.5 & Yes \\
OFD-A5 & 67.5 & Yes \\
TNXOD-B8 & 51.2 & Yes \\
TNXOD-C10 & 36.2 & Yes \\
OFD-D7 & 77.5 & Yes \\
TNXOD-D28 & 40.0 & Yes \\
TNXOD-111 & 38.7 & No \\
TNXSED-25 & 26.2 & \\
Unit Specific Background & & Yes \\
BGCH-5 & 52.5 & Yes \\
BGCH-7 & 68.7 & \\
aReference sediment is from Resurrection Creek, Greenville Co., sc & 'Significance cannot be calculated since the Reference Sediment is the sediment against which all test sediments \\
are compared & &
\end{tabular}

Table 9. Percent Mortality in Definitive Hyalella azteca Toxicity Tests at Locations OFD-A5 and OFD-D7.

\begin{tabular}{lllll} 
& \multicolumn{4}{c}{ Test Concentrations } \\
Location & $\mathbf{1 2 . 5 \%}$ & $\mathbf{2 5 \%}$ & $\mathbf{5 0 \%}$ & $\mathbf{1 0 0 \%}$ \\
\hline OFD-A5 & $69 \%$ & $50 \%$ & $52 \%$ & $67.5 \%$ \\
OFD-D7 & $55 \%$ & $37.5 \%$ & $70 \%$ & $77.5 \%$
\end{tabular}


Table 10. Results of Chironomus tentans Toxicity Tests

\begin{tabular}{lcc} 
Location & Percent Mortality & Significant Mortality $(p=0.05)$ \\
\hline Reference Sediment $^{\mathrm{a}}$ & 33 & $\mathbf{~}^{-}$ \\
OFD-D7 & 50 & Yes \\
BGCH-5 & 85 & Yes
\end{tabular}

\footnotetext{
"Reference sediment is from Resurrection Creek, Greenville Co., SC

'Significance cannot be calculated since the Reference Sediment is the sediment against which all test sediments are compared
}

\subsection{SUMMARYICONCLUSIONS}

The results of the chemical analyses indicate that the locations that were sampled contained elevated levels of mercury and/or uranium, as compared to unit specific background locations. Background concentrations of mercury were $\leq 0.12 \mathrm{mg} / \mathrm{kg}$, while concentrations in the Inner Swamp ranged from 0.2 to $18.4 \mathrm{mg} / \mathrm{kg}$. Background concentrations of uranium were $<5 \mathrm{mg} / \mathrm{kg}$, while concentrations in the Inner Swamp ranged from 62.6 to $254 \mathrm{mg} / \mathrm{kg}$.

The results of toxicity tests conducted on sediments collected from the Inner Swamp and the two unit specific background locations indicated that 9 of the 10 locations that were tested, including the two background locations, were toxic to Hyalella azteca. The toxicity was not related to contaminant concentrations and does not appear to be due to soil type (high clay) and/or lack of organic matter in the sediment material. Additional tests were performed on the location expected have the highest level of mercury and one of the background locations with a different species (Chironomus tentans). The results of these tests also indicated that both sediments were toxic to the test organisms. However, the sediment sample that contained very high levels of mercury and uranium were less toxic than the background reference location, which suggests that the observed toxicity to sediment dwelling invertebrates is not attributable to concentrations of these constituents in the sediment. It was not possible to determine LC50's for mercury or uranium since toxicity was unaffected by dilution. In summary, the results of these three versions of sediment toxicity tests at the TNXOD OU indicated that the toxicity observed in the Inner Swamp sediment is not related to mercury or uranium concentrations.

Even though mercury and uranium concentrations are elevated in the TNX ODU sediments, the metals do not appear to be toxic to sediment biota, as evidenced by the lack of correlation between metal concentrations and sediment toxicity. Mercury binds tightly to organic material and sulfides and in these forms, is not biologically available. Methylated mercury compounds, which are formed by sulfur-reducing bacteria under strongly anoxic conditions, are the most biologically available forms of mercury. The shallow depth of water present at the TNX ODU and periodic drying of the sediments that occurs during periods of low rainfall are not conducive to the formation of methyl mercury. Uranium is very insoluble, which should result in limited mobility and bioavailability in the TNX ODU sediments: 


\subsection{REFERENCES}

ASTM. 1991. Standard Guide for Collection, Storage, Characterization, and Manipulation of Sediments for Toxicological Testing. E1391-90.

ASTM. 1994. Standard Guide for Conducting Sediment Toxicity Tests with Freshwater Invertebrates. E1383-94.

Jones, D.S., G.W. Suter, and R.N.Hull. 1997. Toxicological Benchmarks for Screening Contaminants of Potential Concern foe Effects on Sediment Associated Biota. ES/ER/TM-95/R4, Oak Ridge National Laboratory, Oak Ridge, TN.

Dixon, K.L., V.A. Rogers, S.P. Conner, C.L. Cummins, J.B. Gladden, and J.M. Weber. 1997. Geochemical and Physical Properties of Wetland Soils at the Savannah River Site. WSRC-TR-96-0115.

Kabata-Pendias, A. and H. Pendias. 1992. Trace Metals in Soils and Plants, $2^{\text {nd }}$ edition, pages 146 and 181. CRC Press.

U.S. EPA. 1994. Methods for Measuring the Toxicity and Bioaccumulation of Sedimentassociated Contaminants with Freshwater Invertebrates. EPA/600/R-94-024.

U.S. EPA. 1996. Environmental Investigations, Standard Operating Procedures and Quality Assurance Manual (SOPQAM), United States Environmental Protection Agency (US EPA), Region 4, Athens, GA.

WSRC. 1996. Westinghouse Savannah River Company. Data Summary Report for the RFI/RI Assessment of the TNX Area Operable Unit. ESH-EMS-960504. Savannah River Site, Aiken, SC.

WSRC. 1997. Westinghouse Savannah River Company. WSRC Hydrogeologic Data Collection Procedures Manual 3Q5, Rev. 2, Savannah River Site, Aiken, SC.

WSRC. 1999. Westinghouse Savannah River Company. Data Summary Report for the RFI/RI Assessment of the TNX Outfall Delta. WSRC-TR-99-00034. Savannah River Site, Aiken, SC. 
APPENDIX 1 


\title{
10 DAY AMPHIPOD SEDIMENT TOXICITY TEST
}

\author{
EPA/600/R-94/024 Method 100.1
}

Test Organism: Hyallela azteca

\author{
Client: WSRC \\ Sample Identification: $\quad$ A-4 \\ Test Start Date: $\quad 8 / 4 / 99$
}




\section{DAY AMPHIPOD SEDIMENT TOXICITY TEST}

\begin{tabular}{|l|l|l|}
\hline Lab D\# & Client: WSRC & Start Date: $8 / 4 / 99$ \\
\cline { 2 - 4 } T12306 & Sample Ydentification: A-4 & End Date: 8/14/99 \\
\hline
\end{tabular}

Test System

EPA/600/R-94/024 Method 100.1

The test was set as a Pass/Fail test with a control treatment and one sample treatment. Each treatment replicate consisted of $100 \mathrm{~g}$ of sediment and $175 \mathrm{~mL}$ of dilution water. There were eight replicates for each treatment. Ten test organisms were placed in each.replicate. Sediment from a local stream was used in the control treatment. Test organisms were fed with YCT food. The test was conducted at a temperature of $23^{\circ} \mathrm{C}$ and a light cycle of $16 \mathrm{hr}$ light $/ 8 \mathrm{hr}$ dark. Test vessels were $500 \mathrm{~mL}$ plastic beakers placed in a constant temperature incubator room.

Order: Amphipoda

\section{Test Organisms}

Species: Hyallela azteca

Source: Aquatic Biosystems, Fort Collins, CO

Life Stage: $\quad 7$ to 14 days

$\%$ Mortality During 48 Hr Prior to Test::

Taxonomic Verification: RWK 8/4/99

Culturing: as received

Observations: 'Cultures healthy

Acclimation: None

\begin{tabular}{|l|}
\hline \multicolumn{1}{|c|}{ Control Sediment } \\
Source: Resurrection Creek \\
Collection Date: $8 / 4 / 99$ \\
Preservation: kept at $0-4^{\circ} \mathrm{C}$ in plastic \\
Date/Time Added to Test Chambers: $83 / 99$ \\
Observations: $\quad$ silt,detritus \\
Collection Method: grab \\
Disposal
\end{tabular}

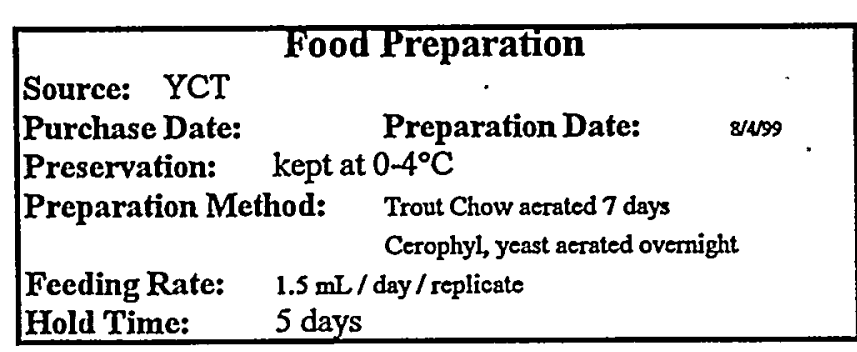

\begin{tabular}{|c|c|c|}
\hline \\
\hline \multicolumn{2}{|c|}{ Type: Surface Water } & \\
\hline Alkalinity & 10.4 & $\mathrm{mg} / \mathrm{L}$ \\
\hline Hardness & 11.4 & $\mathrm{mg} / \mathrm{L}$ \\
\hline Conductivity & 285 & umbos/cm \\
\hline pH: & 7.63 & units \\
\hline $\begin{array}{l}\text { Preparation Method: } \\
\text { Grab sample }\end{array}$ & & \\
\hline
\end{tabular}

Source: A-4

Test Sediment

Collection Date 7/19/99 Homogenized?: yes

Preservation: kept at $0-4^{\circ} \mathrm{C}$ in plastic

Date/Time Added to Test Chambers: $8 / 399$

Observations:

Collection Method: grab

Shipment: overnight

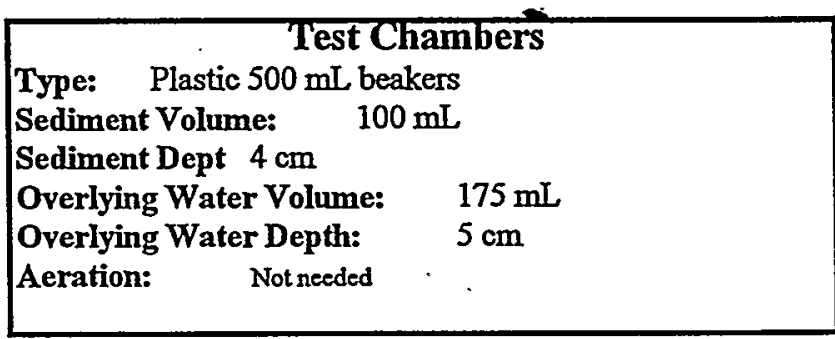




\section{DAY AMPHIPOD SEDIMENT TOXICITY TEST}

\begin{tabular}{|c|c|c|c|}
\hline Lab D\# & WSRC & Start Date: & $8 / 4 / 99$ \\
\hline T12306 & Sample Identification: & End Date: & $8 / 14 / 9$ \\
\hline
\end{tabular}

WATER CHEMISTRY TEST METHODS

Temperature: SM 18th Ed. 2550

Dissolved Oxygen: SM 18th Ed. 4500-O

pH: SM 18th Ed. 4500-H+

Alkalinity: SM 18th Ed. 2320

Hardness: SM 18th Ed. 2340

Conductivity: SM 18th Ed. 2510

\section{WATER CHEMISTRY TEST DATA - OVERLYING WATER}

CONTROL TREATMENT

\begin{tabular}{|c|c|c|c|c|c|c|}
\hline & Temp. & $\mathrm{pH}$ & D.O. & D.O. & Alkalinity & Ammonia \\
\hline Test Day & C & units & $m g / L$ & \% sat. & Initial & Initial \\
\hline Start & 22.3 & 7.63 & 8.0 & $92 \%$ & 10.4 & $<0.20$ \\
\hline 1 & 20.5 & & 7.9 & $87 \%$ & Final & Firal \\
\hline 2 & 22.6 & & 7.6 & $87 \%$ & 49.4 & $<0.1$ \\
\hline 3 & 22.0 & & 7.5 & $86 \%$ & $\mathrm{mgl}$ & $\mathrm{mg} \Omega$ \\
\hline 4 & 20.2 & & 7.8 & $86 \%$ & Cond. & Hardness \\
\hline 5 & 21.5 & & 7.0 & $79 \%$ & Iritial & Initial \\
\hline 6 & 22.2 & & 7.2 & $83 \%$ & 285 & 11.4 \\
\hline 7 & 21.2 & & 6.8 & $76 \%$ & Final & Final \\
\hline 8 & 20.8 & & 6.8 & $75 \%$ & 175.0 & 49.4 \\
\hline 9 & 20.2 & & 8.0 & $88 \%$ & umhos $/ \mathrm{cm}$ & $\mathrm{mg} \Omega$ \\
\hline 10 & 21.0 & 7.46 & 4.9 & $55 \%$ & Aeration: $n$ & \\
\hline
\end{tabular}

SURVIVAL AND GROWTH RESULTS

CONTROL TREATMENT

\begin{tabular}{|c|c|c|c|c|c|c|}
\hline & \multicolumn{3}{|c|}{ \# Organisms } & \multirow[b]{2}{*}{$\begin{array}{c}\% \\
\text { Survival }\end{array}$} & \multirow{2}{*}{$\begin{array}{l}\text { Mean } \\
\text { Length } \\
(\mathrm{mm})\end{array}$} & \multirow{2}{*}{$\begin{array}{l}\text { Standard } \\
\text { Deviation }\end{array}$} \\
\hline & Initial & $\begin{array}{c}\text { Live at } \\
\text { End }\end{array}$ & $\begin{array}{l}\text { Dead } \\
\text { at End }\end{array}$ & & & \\
\hline $\bar{A}$ & 10 & 10 & 0 & $100 \%$ & & \\
\hline $\mathrm{B}$ & 10 & 6 & 4 & $60 \%$ & & \\
\hline $\mathrm{C}$ & 10 & 9 & 1 & $90 \%$ & & \\
\hline D & 10 & 8 & 2 & $80 \%$ & & \\
\hline$E$ & 10 & 10 & 0 & $100 \%$ & & \\
\hline$F$ & 10 & 10 & 0 & $100 \%$ & & \\
\hline $\bar{G}$ & 10 & 5 & 5 & $50 \%$ & & \\
\hline$\overline{\mathrm{H}}$ & 10 & 9 & 1 & $90 \%$ & & \\
\hline Mean & 10.0 & & & $84 \%$ & & \\
\hline
\end{tabular}

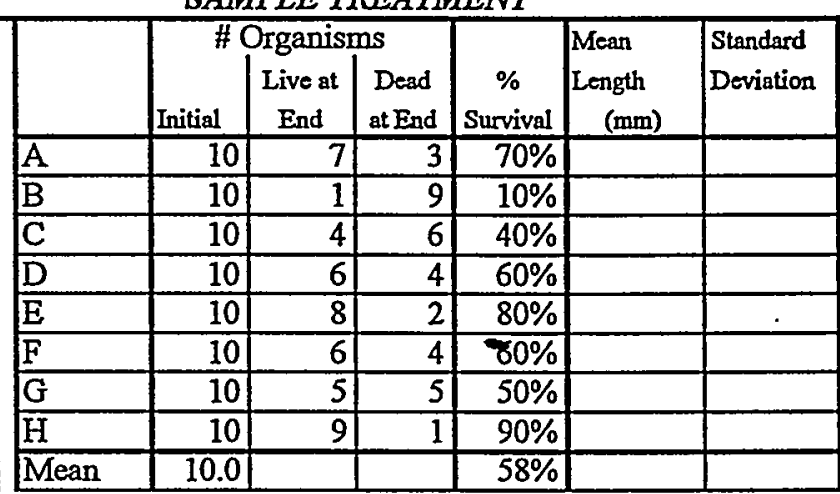




\section{DAY AMPHIPOD SEDIMENT TOXICITY TEST}

\begin{tabular}{|l|l|l|}
\hline Lab ID\# & Client: $\quad$ WSRC & Start Date: 8/4/99 \\
\cline { 2 - 4 } T12306 Sample Identification: A-4 & End Date: $8 / 14 / 99$ \\
\hline
\end{tabular}

\section{RESULTS}

\begin{tabular}{|l|c|}
\hline \multicolumn{2}{|c|}{ SURVIVAL DATA } \\
& Mean of Eight Replicates \\
\cline { 2 - 2 } & Mean $\%$ \\
\hline Control & $83.8 \%$ \\
\hline Sample & $57.5 \%$ \\
\hline
\end{tabular}

\begin{tabular}{|l|l|}
\hline \multicolumn{2}{|c|}{$\begin{array}{c}\text { GROWTH DATA } \\
\text { Mean Length (mm) }\end{array}$} \\
\cline { 2 - 2 } & \\
\hline Control & Mean \\
\hline Sample & NA \\
\hline
\end{tabular}

\section{STATISTICAL ANALYSES}

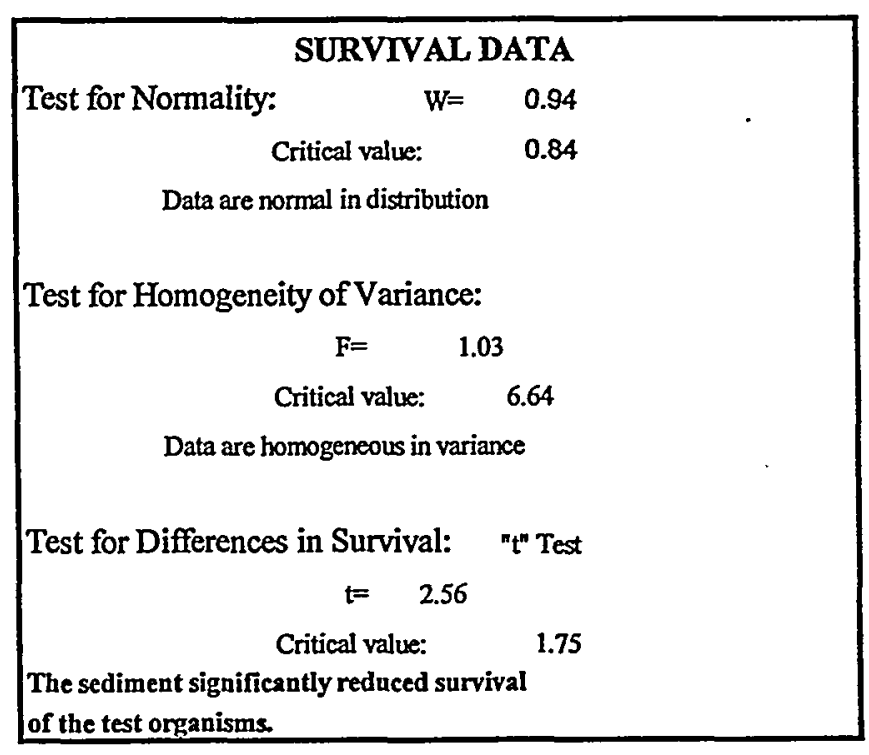

\begin{tabular}{cc}
\hline \multicolumn{3}{c}{ GROWTH DATA } \\
Test for Normality: & W= NA \\
NA &
\end{tabular}

Test for Homogeneity of Variance:

$\begin{array}{cr}F= & \text { NA } \\ \text { Critical value: } & 6.635\end{array}$

NA

Test for Differences in Survival: " $\mathrm{t}^{\mathrm{n}}$ Test

$$
\begin{aligned}
& \cdot t=N A \\
& \text { Critical value: }
\end{aligned}
$$

\section{COMMENTS}

None

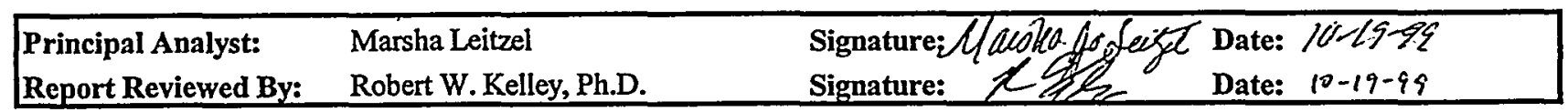

\section{CONCLUSIONS}

The sediment reduces the survival of the test organisms. 


\title{
10 Day Chronic Definitive Sediment Toxicity Test Method: EPA/600/R-94/024 100.1
}

\author{
Test Organism: Hyallela azteca
}

Facility: WSRC

Sample ID: A-5

$$
\begin{aligned}
& \text { County: Aiken } \\
& \text { NPDES\#: } \\
& \text { ETT\#: T12305 }
\end{aligned}
$$

Sample ID: August 4, 1999 


\section{CHRONIC DEFINITIVE SEDIMENT 10 DAY SURVIVAL TEST}

\begin{tabular}{|l|l|l|ll|}
\hline Lab W\# & Client: & \multicolumn{1}{|c|}{ WSRC } & Start Date: & 8/4/99 \\
\cline { 2 - 6 } T12304 & Sample Identification: & D-7 & End Date: & $8 / 14 / 99$ \\
\hline
\end{tabular}

\section{Test System}

\section{EPA/600/R-94/024 Method 100.1}

The test was set as a Definitive test with a control treatment and four sample treatments. Each treatment replicate consisted of $100 \mathrm{~g}$ of sediment and $175 \mathrm{~mL}$ of dilution water. There were four replicates for each treatment. Ten test organisms were placed in each replicate. Sediment from a local stream was used in the control treatment. Test organisms were fed with YCT food. The test was conducted at a temperature of $23^{\circ} \mathrm{C}$ and a light cycle of $16 \mathrm{hr}$ light/8 hr dark. Test vessels were $500 \mathrm{~mL}$ plastic beakers placed in a constant temperature incubator room.

Order: Amphipoda

\section{Test Organisms}

Species: Hyallela azteca

Source: Aquatic Biosystems, Fort Collins, CO

Life Stage: 7 to 14 days

$\%$ Mortality During 48 Hr Prior to Test::

Taxonomic Verification: RWK 8/4/99

Culturing: as received

Observations: Cultures healthy

Acclimation: None

\begin{tabular}{l}
\hline \multicolumn{1}{|c|}{ Control Sediment } \\
Source: BGCH7 \\
Collection Date: 7/19/99 \\
Preservation: kept at $0-4^{\circ} \mathrm{C}$ in plastic \\
Date/Time Added to Test Chambers: \\
Observations: \\
Collection Method: $\quad$ grab \\
Disposal:
\end{tabular}

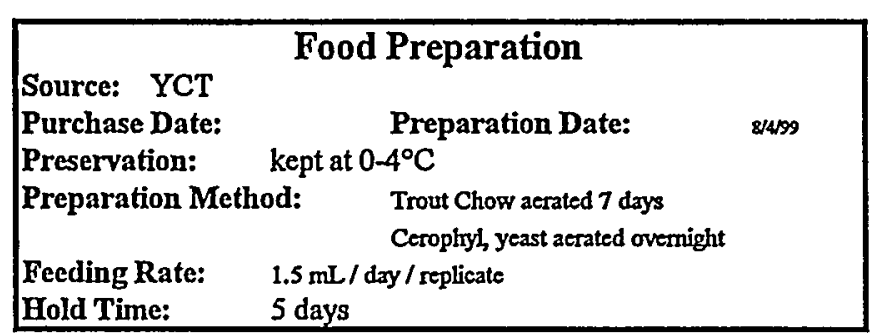

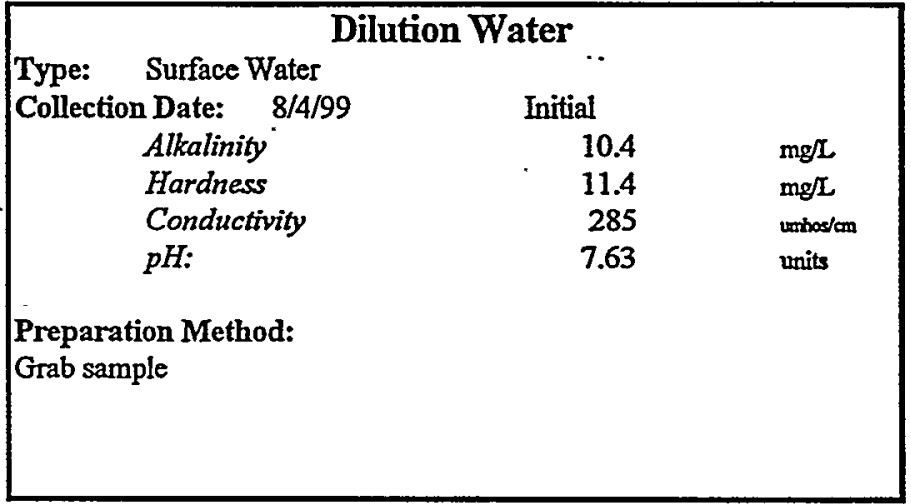

Source: A-5

\section{Test Sediment}

Collection Date: 7/19/99 Homogenized?: yes

Preservation: kept at $0-4^{\circ} \mathrm{C}$ in plastic

Date/Time Added to Test Chambers:

Observations:

Collection Method: grab

Shipment: overnight

Type: Plastic $500 \mathrm{~mL}$ beakers

\section{Test Chambers}

Sediment Volume: $\quad 100 \mathrm{~mL}$

Sediment Depth: $4 \mathrm{~cm}$

Overlying Water Volume: $\quad 175 \mathrm{~mL}$

Overlying Water Depth: $\quad .5 \mathrm{~cm}$ 


\section{CHRONIC DEFINITIVE SEDIMENT 10 DAY SURVIVAL TEST Survival Results}

Client: WSRC
Sample Identification: A-5
Test Date:
\begin{tabular}{|c|c|c|c|c|c|c|}
\hline Survival in & \multicolumn{7}{c|}{ Control sediment (BGCH7) } & \\
\hline Day & Rep. A & Rep B & Rep. C & Rep. D & & Mean \% \\
\hline 0 & 10 & 10 & 10 & 10 & & $100 \%$ \\
\hline & & & & & & \\
\hline & & & & & & \\
\hline & & & & & & \\
\hline & & & & & & \\
\hline & & & & & & \\
\hline & & & & & & \\
\hline 10 & 1 & 1 & 4 & 6 & & $30 \%$ \\
\hline \# Dead & 9 & 9 & 6 & 4 & & $70 \%$ \\
\hline \# Lost & 0 & 0 & 0 & 0 & & $0 \%$ \\
\hline
\end{tabular}

$\begin{array}{ll}\text { County: } & \text { Aiken } \\ \text { NPDES\#: } & \\ \text { ETT \#: } & \text { T12305 }\end{array}$

\begin{tabular}{|c|c|c|c|c|c|c|}
\hline Survival at & \multicolumn{7}{|c|}{$12.5 \%$ sediment A-5 w/ 87.5\% sediment BGCH7 } & Mean \% \\
\hline Day & Rep. A & Rep B & Rep. C & Rep. D & & $100 \%$ \\
\hline 0 & 10 & 10 & 10 & 10 & & \\
\hline & & & & & & \\
\hline & & & & & & \\
\hline & & & & & & \\
\hline & & & & & & \\
\hline & & & & & & \\
\hline 10 & 2 & 7 & 2 & 2 & & $33 \%$ \\
\hline \# Dead & 8 & 3 & 8 & 8 & & $68 \%$ \\
\hline \# Lost & 0 & 0 & 0 & 0 & & $0 \%$ \\
\hline
\end{tabular}

\begin{tabular}{|c|c|c|c|c|c|c|}
\hline \multicolumn{7}{|l|}{ Survival at } \\
\hline Day & Rep. A & Rep B & Rep. C & Rep. D & & Mean \% \\
\hline 0 & 10 & 10 & 10 & 10 & & $100 \%$ \\
\hline & & & & & & \\
\hline & & & & & & \\
\hline & & & & & & \\
\hline & & & & & & \\
\hline & & & & & & \\
\hline 10 & 3 & 6 & 2 & 9 & & $50 \%$ \\
\hline \# Dead & 7 & 4 & 8 & 1 & & $50 \%$ \\
\hline \# Lost & 0 & 0 & 0 & 0 & & $0 \%$ \\
\hline
\end{tabular}

\begin{tabular}{|c|c|c|c|c|c|c|}
\hline \multicolumn{7}{|l|}{ Survival at } \\
\hline Day & Rep. A & Rep B & Rep. C & Rep. D & & Mean \% \\
\hline 0 & 10 & 10 & 10 & 10 & & $100 \%$ \\
\hline & & & & & & \\
\hline & & & & & & \\
\hline & & & & & & \\
\hline & & & & & & \\
\hline & & & & & & \\
\hline & & & & & & \\
\hline 10 & 6 & 0 & 2 & 5 & & $33 \%$ \\
\hline \#Dead & 4 & 10 & 8 & 5 & & $68 \%$ \\
\hline \#Lost & 0 & 0 & 0 & 0 & & $0 \%$ \\
\hline
\end{tabular}

\begin{tabular}{|c|l|l|l|l|l|l|}
\hline \multicolumn{5}{|l|}{ Survival at } & \multicolumn{1}{l|}{} \\
\hline Day & Rep. A & Rep B & Rep. C & Rep. D & & Mean \% \\
\hline 0 & & & & & & \\
\hline 1 & & & & & & \\
\hline 2 & & & & & & \\
\hline 3 & & & & - & & \\
\hline 4 & & & & & & \\
\hline 5 & & & & & & \\
\hline 6 & & & & & & \\
\hline 7 & & & & & & \\
\hline \#Dead & & & & & & \\
\hline \# Lost & & & & & & \\
\hline
\end{tabular}




\section{CHRONIC DEFINITIVE SEDIMENT 10 DAY SURVIVAL TEST Statistical Analyses - Survival}

Client: WSRC

Sample Identification

Test Date:

August 4, 1999

\begin{tabular}{llll}
\hline Test for Normality & & \\
& & & \\
Test Used: Shapiro-Wilks & W= & 0.953 \\
& & critical & 0.884
\end{tabular}

The data are normal in distribution

\section{Test for Homogeneity of Variance}

Test Used: Bartlett's Test

$\mathrm{B}=\quad 0.56$

The data are homogeneous in variance

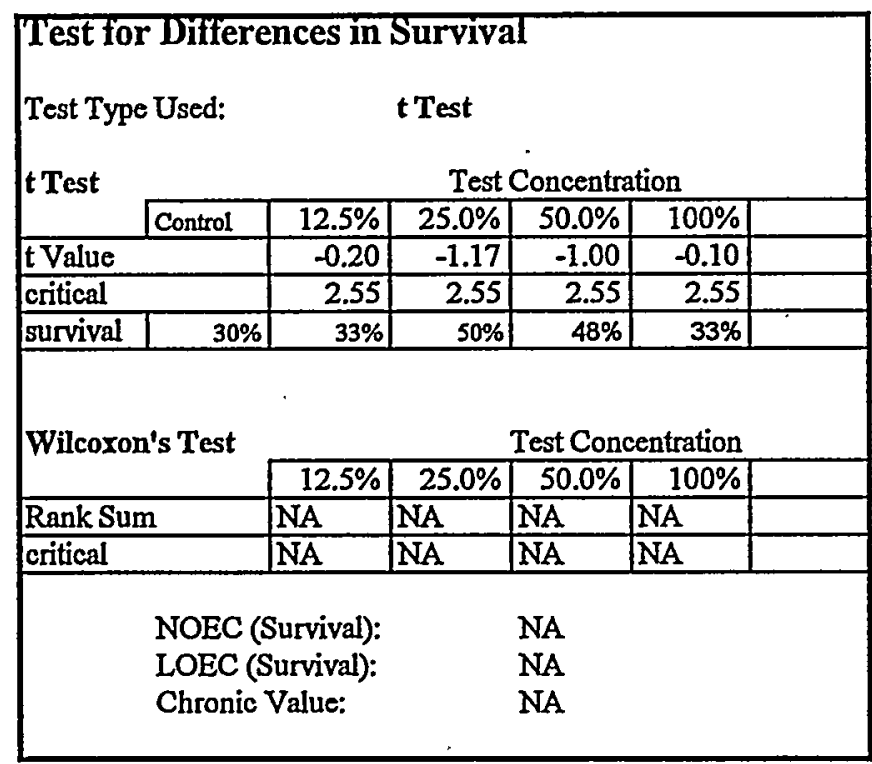

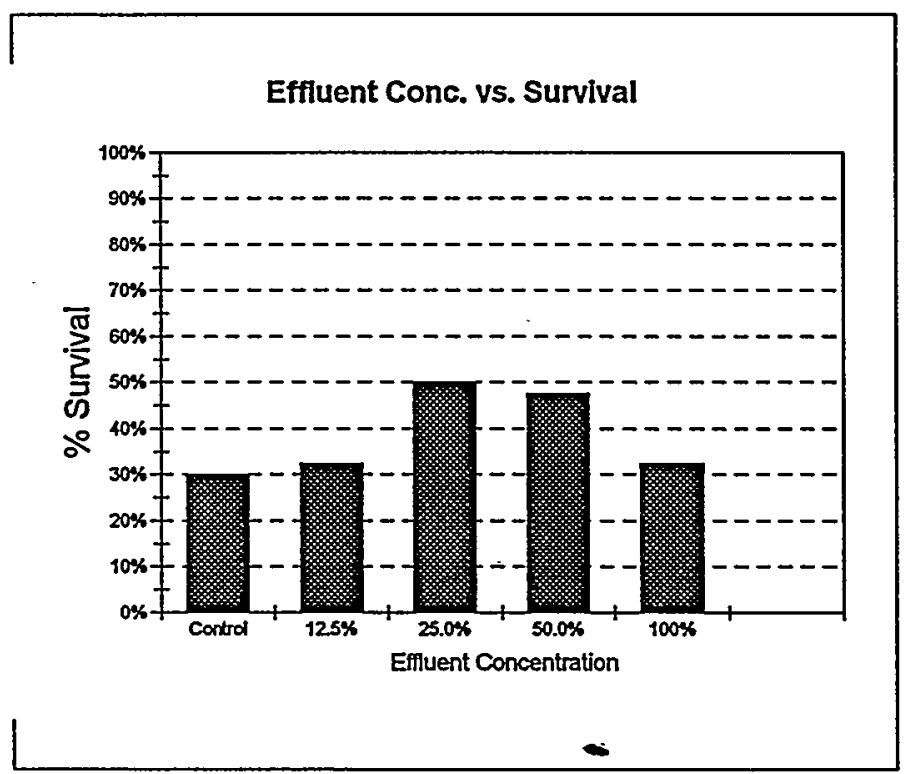

Summary Both the control sediment and the test sediment reduced survival.

Survival in $100 \%$ control sediment was not significantly less. than in the control sediment. 


\section{CHRONIC DEFINITIVE SEDIMENT 10 DAY SURVIVAL TEST Water Chemistry}

Client: WSRC

Sample Identification: A-5

Test Date: $\quad$ August 4, 1999

Control Sediment

\begin{tabular}{|l|r|r|r|r|l|l|}
\hline & \multicolumn{1}{|c|}{ Alkal. } & Hard. & Cond. & NH3 & & \\
\hline & $\mathrm{mg} / \mathrm{L}$ & $\mathrm{mg} / \mathrm{L}$ & umhos & $\mathrm{mg} \mathcal{L}$ & & \\
\hline Initial & 10.4 & 11.4 & 285 & $<0.1$ & & \\
\hline Day 10 & 88.2 & 49.4 & 175 & $<0.2$ & & \\
\hline
\end{tabular}

Temperature

\begin{tabular}{|c|c|c|c|c|c|c|}
\hline & \multicolumn{6}{|c|}{ Test Concentration } \\
\hline & Control & $12.50 \%$ & $25.00 \%$ & $50.00 \%$ & & $100 \%$ \\
\hline Initial & 22.3 & & 22.3 & & & 22.3 \\
\hline Day 1 & 20.2 & & 20.5 & & & 20.5 \\
\hline Day 2 & 22.6 & & 22.2 & & २ & 22.6 \\
\hline Day 3 & 21.1 & & 22.0 & & & 22.0 \\
\hline Day 4 & 20.2 & & 20.2 & א/ & & 20.2 \\
\hline Day 5 & 21.5 & & 21.5 & 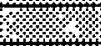 & & 21.5 \\
\hline Day 6 & 22.2 & & 22.2 & $\%$ & & 22.2 \\
\hline Day 7 & 21.2 & & 21.2 & \% & 2 & 20.8 \\
\hline Day 8 & 20.8 & & 20.8 & & $\%$ & 19.8 \\
\hline Day 9 & 19.7 & & 19.8 & $\%$ & & 21.0 \\
\hline Day 10 & 21.0 & & 21.0 & & & 21.0 \\
\hline
\end{tabular}

Test Sediment

\begin{tabular}{|r|r|r|r|r|r|r|}
\hline & Alkal. & Hard. & Cond. & NH3 & & \\
\hline & $\mathrm{mgl}$ & $\mathrm{mg} / \mathrm{L}$ & umhos & $\mathrm{mgl}$ & & \\
\hline Initial & 10.4 & 11.4 & 285 & $<0.1$ & & \\
\hline Day 10 & 10.7 & 3.8 & 41 & 0.13 & & \\
\hline
\end{tabular}

Dissolved Oxygen

\begin{tabular}{|c|c|c|c|c|c|c|}
\hline & \multicolumn{6}{|c|}{ Test Concentration } \\
\hline & Control & $12.50 \%$ & $25.00 \%$ & $50.00 \%$ & & $100 \%$ \\
\hline Initial & 8.0 & & $\overline{8.0}$ & m & & 8.0 \\
\hline Day 1 & 8.0 & & 7.6 & & & 7.7 \\
\hline Day 2 & 7.2 & & 8.0 & & & 8.0 \\
\hline Day 3 & 7.5 & & 8.0 & & & 8.0 \\
\hline Day 4 & 7.2 & & 7.4 & & \% & 7.4 \\
\hline Day 5 & 7.2 & & 7.7 & & & 7.8 \\
\hline Day 6 & 7.5 & & 7.4 & & & 7.4 \\
\hline Day 7 & 7.1 & & 7.2 & & & 7.2 \\
\hline Day 8 & 7.0 & & 7.3 & & & 7.3 \\
\hline Day 9 & 7.8 & & 7.8 & & $\% \%$ & 7.7 \\
\hline Day 10 & 7.7 & & & & & 8.1 \\
\hline
\end{tabular}

Test Results Reviewed and Approved By: 


\title{
10 DAY AMPHIPOD SEDIMENT TOXICITY TEST
}

\author{
EPA/600/R-94/024 Method 100.1
}

Test Organism: Hyallela azteca

\author{
Client: WSRC \\ Sample Identification: A-11 \\ Test Start Date: $\quad 8 / 4 / 99$
}




\section{DAY AMPHIPOD SEDIMENT TOXICITY TEST}

\begin{tabular}{|c|c|c|c|}
\hline \multirow{2}{*}{$\begin{array}{l}\text { Lab D\#\# } \\
\text { T12309 }\end{array}$} & WSRC & Start Date: & $8 / 4 / 99$ \\
\hline & Sample Identification: & End Date: & $8 / 14 / 99$ \\
\hline
\end{tabular}

\section{Test System \\ EPA/600/R-94/024 Method 100.1}

The test was set as a Pass/Fail test with a control treatment and one sample treatment. Each treatment replicate consisted of $100 \mathrm{~g}$ of sediment and $175 \mathrm{~mL}$ of dilution water. There were eight replicates for each treatment. Ten test organisms were placed in each replicate. Sediment from a local stream was used in the control treatment. Test organisms were fed with YCT food. The test was conducted at a temperature of $23^{\circ} \mathrm{C}$ and a light cycle of $16 \mathrm{hr}$ light/8 hr dark. Test vessels were $500 \mathrm{~mL}$ plastic beakers placed in a constant temperature incubator room.

Order: Amphipoda

Species: Hyallela azteca

Source: Aquatic Biosystems, Fort Collins, CO

Life Stage: $\quad 7$ to 14 days

\% Mortality During 48 Hr Prior to Test:: $\quad 0 \%$

Taxonomic Verification: RWK 8/4/99

Culturing: as received

Observations: Cultures healthy

Acclimation: None

Source: Resurrection Creek

\section{ControISediment}

Collection Date: 8/4/99

Preservation: kept at $0-4^{\circ} \mathrm{C}$ in plastic

Date/Time Added to Test Chambers: $8 / 3 / 9 s$

Observations: silt and organic

Collection Method: grab

Disposal

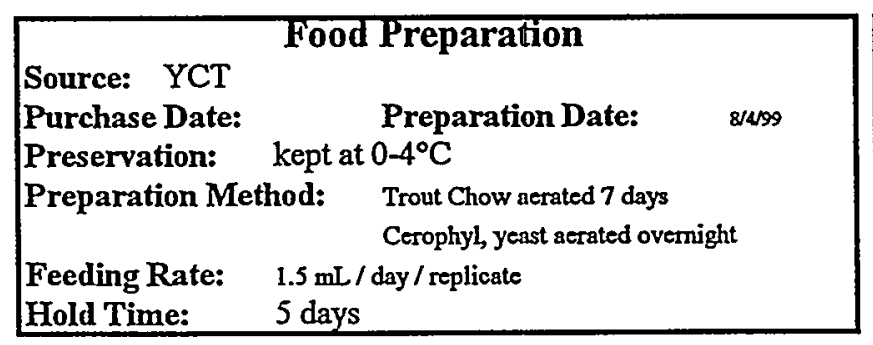

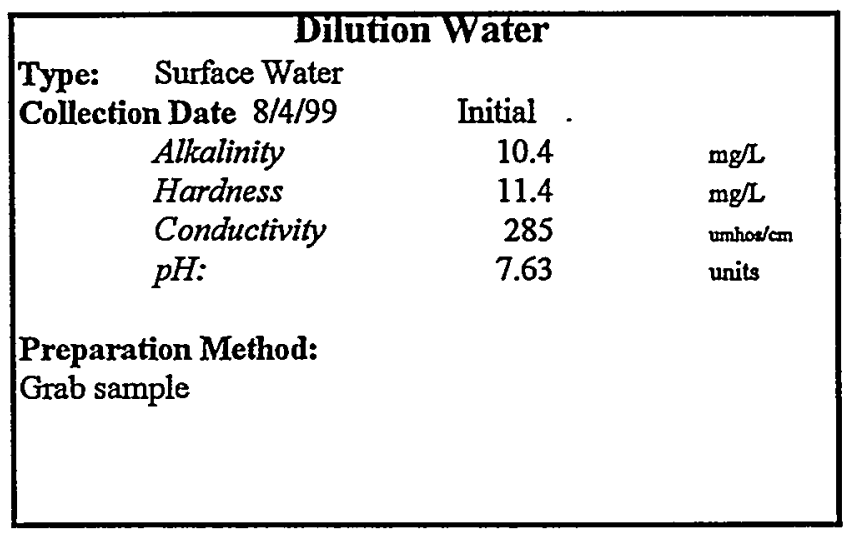

Source: I-11

Collection Date 7/16/99 Homogenized?: yes

Preservation: kept at $0-4^{\circ} \mathrm{C}$ in plastic

Date/Time Added to Test Chambers: $8 / 1 / 99$

Observations:

Collection Method: grab

Shipment: ovemight

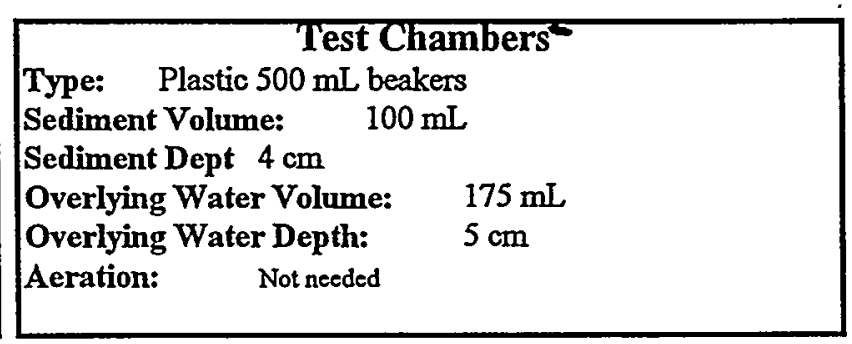




\section{DAY AMPHIPOD SEDIMENT TOXICITY TEST}

\begin{tabular}{|l|l|l|}
\hline Lab D\#\# & Client: $\quad$ WSRC & Start Date: $8 / 4 / 99$ \\
\cline { 2 - 3 } T12309 & Sample Identification: A-11 & End Date: 8/14/99 \\
\hline
\end{tabular}

\section{WATER CHEMISTRY TEST METHODS}

Temperature: SM 18th Ed. 2550

Dissolved Oxygen: SM 18th Ed. 4500-O

pH: SM 18th Ed. 4500-H+
Alkalinity: SM 18th Ed. 2320

Hardness: SM 18th Ed. 2340

Conductivity: SM 18th Ed. 2510

\section{WATER CHEMISTRY TEST DATA - OVERLYING WATER}

CONTROL TREATMENT

\begin{tabular}{|c|c|c|c|c|c|c|}
\hline & Temp. & $\mathrm{pH}$ & D.o. & D.o. & Alkalinity & Ammonia \\
\hline Test Day & c & units & $\mathrm{mg} / \mathrm{L}$ & $\%$ sat. & Initial & Initial \\
\hline Start & 22.3 & 7.63 & 8.0 & $92 \%$ & 10.4 & $<0.20$ \\
\hline$\overline{1}$ & 20.5 & & 7.9 & $87 \%$ & Final & Final \\
\hline 2 & 22.6 & & 7.6 & $87 \%$ & 88.2 & $<0.1$ \\
\hline 3 & 22.0 & & 7.5 & $86 \%$ & $\mathrm{mgr}$ & $\mathrm{mgh}$ \\
\hline 4 & 20.2 & & 7.8 & $86 \%$ & Cond. & Hardness \\
\hline 5 & 21.5 & & 7.0 & $79 \%$ & Initial & Iritital \\
\hline 6 & 22.2 & & 7.2 & $83 \%$ & 285 & 11.4 \\
\hline 7 & 21.2 & & 6.8 & $76 \%$ & Final & Fral \\
\hline 8 & 20.8 & & 6.8 & $75 \%$ & 175.0 & 49.4 \\
\hline 9 & 20.2 & & 8.0 & $88 \%$ & umhos $/ \mathrm{cm}$ & $\mathrm{mgh}$ \\
\hline$\overline{10}$ & 21.0 & 7.46 & 4.9 & $55 \%$ & Actalio & \\
\hline
\end{tabular}

SAMPLE TREATMENT

\begin{tabular}{|c|c|c|c|c|c|c|}
\hline & Temp. & $\mathrm{pH}$ & D.O. & D.O. & Alkalinity & Ammonia \\
\hline Test Day & C & units & $m g / L$ & $\%$ sat. & Initial & Initial \\
\hline Start & $\overline{22.3}$ & 7.63 & 8.0 & & 10.4 & $<0.20$ \\
\hline 1 & $\overline{20.1}$ & & 8.0 & $88 \%$ & Final & Frol \\
\hline 2 & 22.6 & & 8.0 & $92 \%$ & 7.6 & $<0.1$ \\
\hline 3 & 21.2 & & 7.7 & $87 \%$ & $\mathrm{mgh}$ & $\mathrm{mgr}$ \\
\hline 4 & $\overline{20.2}$ & & 7.5 & $82 \%$ & Cond. & Hardness \\
\hline 5 & 21.5 & & 7.2 & $81 \%$ & Initiol & Initial \\
\hline 6 & 22.2 & & 7.6 & $87 \%$ & 285 & 11.4 \\
\hline$\overline{7}$ & $\overline{21.2}$ & & 7.3 & $82 \%$ & Final & Frnal \\
\hline$\overline{8}$ & 20.8 & & 7.2 & $79 \%$ & 44.0 & 9.5 \\
\hline 9 & 20.3 & & 7.7 & $85 \%$ & umhos $/ \mathrm{cm}$ & $\mathrm{mgr}$ \\
\hline 10 & 21.0 & 7.07 & 7.8 & $88 \%$ & \multicolumn{2}{|l|}{ Aeratio } \\
\hline
\end{tabular}

SURVIVAL AND GROWTH RESULTS

CONTROL TREATMENT

\begin{tabular}{|c|c|c|c|c|c|c|}
\hline & Initial & $\begin{array}{l}\text { rganism } \\
\text { Live at } \\
\text { End }\end{array}$ & $\begin{array}{l}\text { IS } \\
\text { Dead } \\
\text { at End }\end{array}$ & $\begin{array}{c}\% \\
\text { Survival }\end{array}$ & $\begin{array}{l}\text { Mean } \\
\text { Length } \\
\text { (mm) }\end{array}$ & $\begin{array}{l}\text { Standard } \\
\text { Deviation }\end{array}$ \\
\hline A & 10 & 10 & 0 & $100 \%$ & & \\
\hline$B$ & 10 & 6 & 4 & $60 \%$ & & \\
\hline $\mathrm{C}$ & 10 & 9 & 1 & $90 \%$ & & \\
\hline $\mathrm{D}$ & 10 & 8 & 2 & $80 \%$ & & \\
\hline $\mathrm{E}$ & 10 & 10 & 0 & $100 \%$ & & \\
\hline $\mathbf{F}$ & 10 & 10 & 0 & $100 \%$ & & \\
\hline $\mathrm{G}$ & .10 & 5 & 5 & $50 \%$ & & \\
\hline $\mathrm{H}$ & 10 & 9 & 1 & $90 \%$ & & \\
\hline Mean & 10.0 & & & $84 \%$ & & \\
\hline
\end{tabular}

SAMPLE TREATMENT

\begin{tabular}{|l|r|r|r|r|l|l|}
\hline & \multicolumn{9}{|c|}{$\begin{array}{c}\text { \# Organisms } \\
\text { Initial }\end{array}$} & $\begin{array}{c}\text { Live at } \\
\text { End }\end{array}$ & $\begin{array}{c}\text { Dead } \\
\text { at End }\end{array}$ & $\begin{array}{l}\text { Mean } \\
\text { Survival }\end{array}$ & $\begin{array}{l}\text { Length } \\
\text { (mm) }\end{array}$ & Deviation \\
\hline A & 10 & 6 & 4 & $60 \%$ & & \\
\hline B & 10 & 6 & 4 & $60 \%$ & & \\
\hline C & 10 & 8 & 2 & $80 \%$ & & \\
\hline D & 10 & 4 & 6 & $40 \%$ & & \\
\hline E & 10 & 9 & 1 & $90 \%$ & & \\
\hline F & 10 & 5 & 5 & $50 \%$ & & \\
\hline G & 10 & 7 & 3 & $70 \%$ & & \\
\hline H & 10 & 4 & 6 & $40 \%$ & & \\
\hline Mean & 10.0 & & & $61 \%$ & & \\
\hline
\end{tabular}




\section{DAY AMPHIPOD SEDIMENT TOXICITY TEST}

\begin{tabular}{|c|c|c|c|}
\hline \multirow{2}{*}{\begin{tabular}{|l} 
Lab ID\# \\
T12309
\end{tabular}} & WSRC & Start Date: & $8 / 4 / 99$ \\
\hline & Sample Identification: & End Date: & $8 / 14 / 99$ \\
\hline
\end{tabular}

\section{RESULTS}

\begin{tabular}{|l|r|}
\hline \multicolumn{2}{|c|}{$\begin{array}{c}\text { SURVIVAL DATA } \\
\text { Mean of Eight Replicates }\end{array}$} \\
\cline { 2 - 2 } & Mean $\%$ \\
\hline Control & $83.8 \%$ \\
\hline Sample & $61.3 \%$ \\
\hline
\end{tabular}

\begin{tabular}{|l|l|}
\hline \multicolumn{2}{|c|}{$\begin{array}{r}\text { GROWTH DATA } \\
\text { Mean Length }(\mathrm{mm})\end{array}$} \\
\cline { 2 - 2 } & \multicolumn{1}{|c|}{ Mean } \\
\hline Control & NA \\
\hline Sample & NA \\
\hline
\end{tabular}

\section{STATISTICAL ANALYSES}

\begin{tabular}{|ccc|}
\hline \multicolumn{3}{|c}{ SURVIVAL DATA } \\
Test for Normality: $\quad$ W= & 0.95 \\
Critical value: & 0.84 \\
Data are normal in distribution &
\end{tabular}

Test for Homogeneity of Variance:

$$
\begin{array}{cr}
F= & 2.06 \\
\text { Critical value: } & 6.64
\end{array}
$$

Data are homogeneous in variance

Test for Differences in Survival: $\quad "{ }^{n}$ "Test

$$
t=2.58
$$

Critical value: 1.75

The sediment significantly reduced survival of the test organisms.

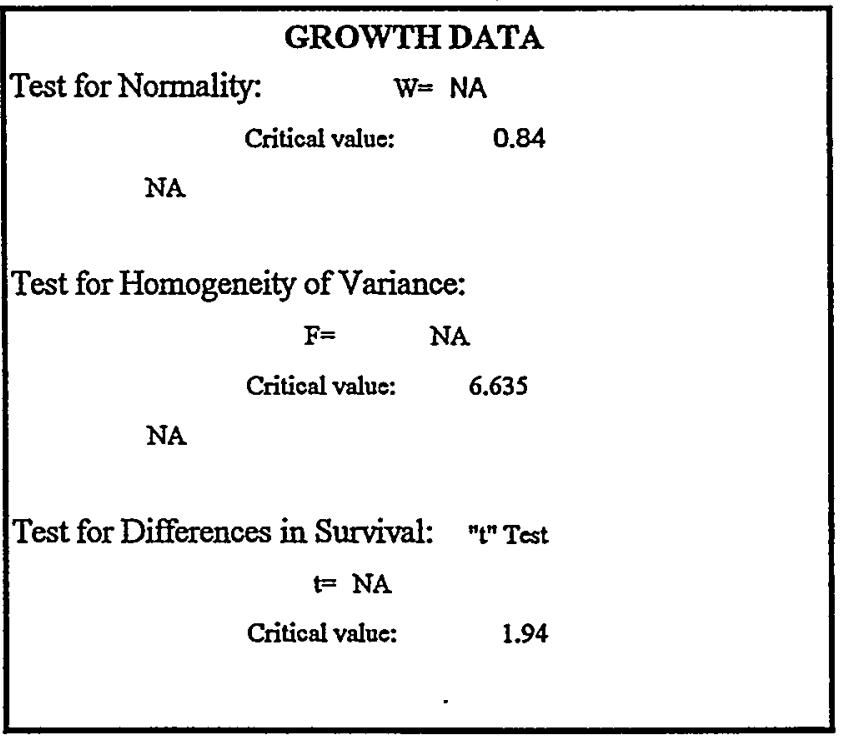

$\infty$

\section{COMMENTS}

None

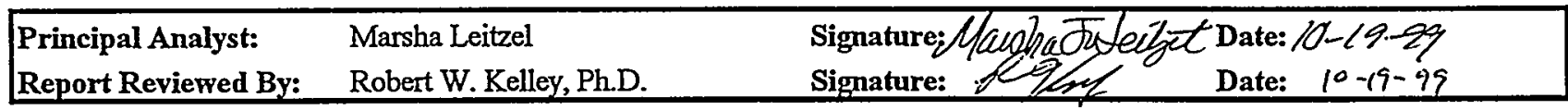

\section{CONCLUSIONS}

The sediment reduces survival of the test organisms. 


\title{
10 DAY AMPHIPOD SEDIMENT TOXICITY TEST
}

EPA/600/R-94/024 Method 100.1

Test Organism: Hyallela azteca

\author{
Client: WSRC \\ Sample Identification: $\quad$ B-8
}

Test Start Date: $\quad 8 / 4 / 99$ 
WSRC-TR-99-00380

\section{DAY AMPHIPOD SEDIMENT TOXICITY TEST}

\begin{tabular}{|l|l|l|}
\hline Lab ID\# & Client: $\quad$ WSRC & Start Date: $8 / 4 / 99$ \\
\cline { 2 - 4 } T12313 & Sample Identification: B-8 & End Date: $8 / 14 / 99$ \\
\hline
\end{tabular}

\section{Test System}

EPA/600/R-94/024 Method 100.1

The test was set as a Pass/Fail test with a control treatment and one sample treatment. Each treatment replicate consisted of $100 \mathrm{~g}$ of sediment and $175 \mathrm{~mL}$ of dilution water. There were eight replicates for each treatment. Ten test organisms were placed in each replicate. Sediment from a local stream was used in the control treatment. Test organisms were fed with YCT food. The test was conducted at a temperature of $23^{\circ} \mathrm{C}$ and a light cycle of $16 \mathrm{hr}$ light/ $8 \mathrm{hr}$ dark. Test vessels were $500 \mathrm{~mL}$ plastic beakers placed in a constant temperature incubator room.

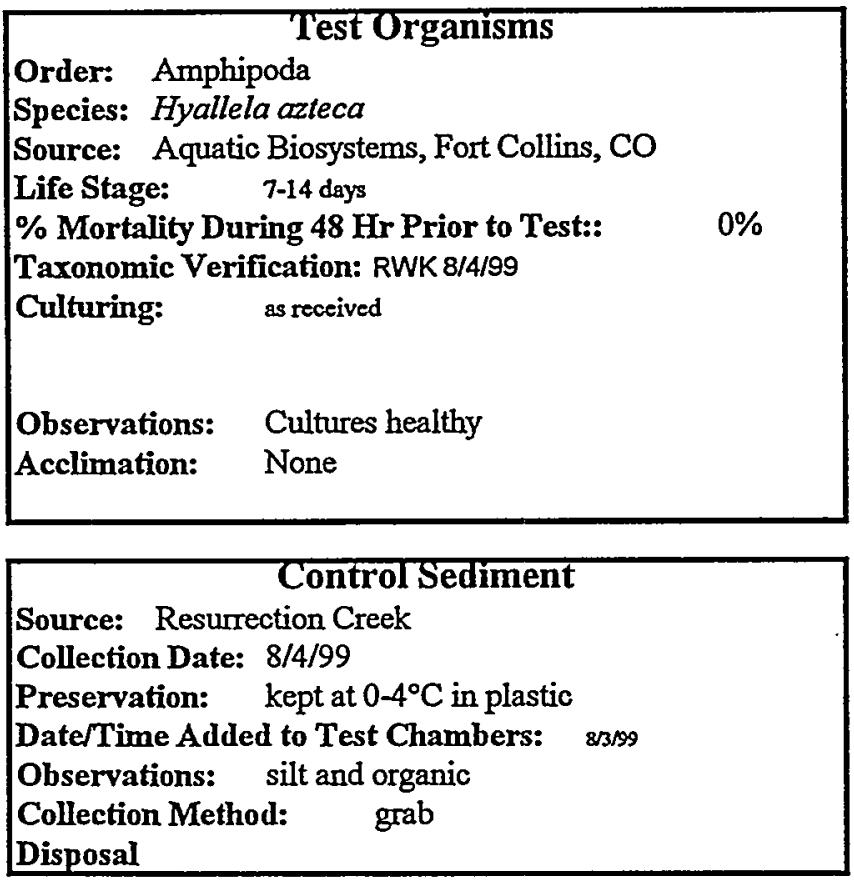

\begin{tabular}{|ccl|}
\hline \multicolumn{3}{|c|}{ Dilution Water } \\
Type: $\quad$ Surface Water & & \\
Collection Date 8/4/99 & Initial & \\
Alkalinity & 10.4 & $\mathrm{mg/L}$ \\
Hardness & 11.4 & $\mathrm{mg} / \mathrm{L}$ \\
Conductivity & 285 & umhosctom \\
pH: & 7.63 & units \\
& & \\
Preparation Method: & & \\
Grab sample & & \\
& \\
\end{tabular}

Source: B-8

Collection Date 7/16/99 Homogenized?: yes

Preservation: kept at $0-4^{\circ} \mathrm{C}$ in plastic

Date/Time Added to Test Chambers: 83/9s

Observations:

Collection Method: grab

Shipment: overnight

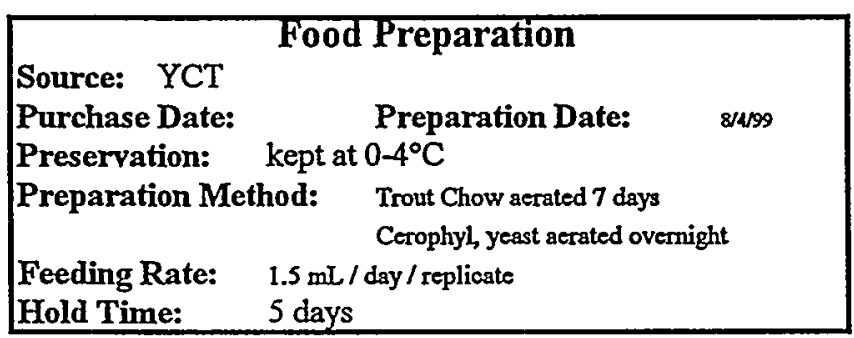

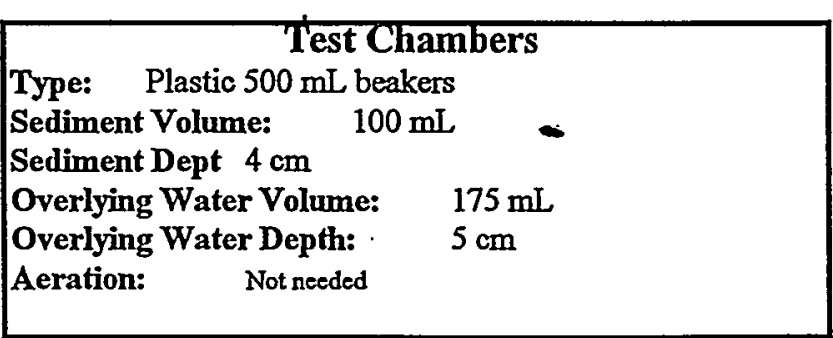




\section{DAY AMPHIPOD SEDIMENT TOXICITY TEST}

\begin{tabular}{|l|l|l|}
\hline Lab ID\# & Client: $\quad$ WSRC & Start Date: $8 / 4 / 99$ \\
\cline { 2 - 4 } T12313 & Sample Identification: B-8 & End Date: $8 / 14 / 99$ \\
\hline
\end{tabular}

\section{WATER CHEMISTRY TEST METHODS}

Temperature: SM 18th Ed. 2550

Dissolved Oxygen: SM 18th Ed. 4500-O

pH: SM 18th Ed. 4500-H+
Alkalinity: SM 18th Ed. 2320

Hardness: SM 18th Ed. 2340

Conductivity: SM 18th Ed. 2510

WATER CHEMISTRY TEST DATA - OVERLYING WATER

CONTROL TREATMENT

\begin{tabular}{|c|c|c|c|c|c|c|}
\hline & Temp. & pH & D.o. & D.O. & Alkalinity & Ammonia \\
\hline Test Day & c & units & $\mathrm{mg} / \mathrm{L}$ & $\%$ sat. & Initial & Initial \\
\hline Start & 22.3 & 7.63 & 8.0 & $92 \%$ & 10.4 & $<0.20$ \\
\hline$\overline{1}$ & 20.5 & & 7.9 & $\overline{87 \%}$ & Final & Fral \\
\hline 2 & 22.6 & & 7.6 & $87 \%$ & 88.2 & $<0.1$ \\
\hline 3 & 22.0 & & 7.5 & $86 \%$ & $\mathrm{mg} \Omega$ & $\mathrm{mgn}$ \\
\hline 4 & 20.2 & & $\overline{7.8}$ & $86 \%$ & Cond. & Hardness \\
\hline 5 & 21.5 & & 7.0 & $79 \%$ & Intitial & Initital \\
\hline 6 & 22.2 & & $\overline{7.2}$ & $83 \%$ & 285 & 11.4 \\
\hline 7 & 21.2 & & 6.8 & $76 \%$ & Fimal & Final \\
\hline 8 & 20.8 & & 6.8 & $75 \%$ & 175.0 & 49.4 \\
\hline 9 & 20.2 & & 8.0 & $88 \%$ & umhos $/ \mathrm{cm}$ & $\mathrm{mgn}$ \\
\hline 10 & 21.0 & 7.46 & 4.9 & $55 \%$ & Acration: & \\
\hline
\end{tabular}

SAMPLE TREATMENT

\begin{tabular}{|c|c|c|c|c|c|c|}
\hline & Temp. & $\mathrm{pH}$ & D.o. & D.o. & Alkalinity & Ammonia \\
\hline Test Day & C & units & $\mathrm{mg} / \mathrm{L}$ & $\%$ sat. & Initial & Initial \\
\hline Start & 22.3 & 7.63 & 8.0 & & 10.4 & $<0.20$ \\
\hline 1 & 20.6 & & 8.0 & $88 \%$ & Finel & Final \\
\hline 2 & 22.6 & & 7.9 & $91 \%$ & 7.2 & $<0.1$ \\
\hline 3 & 22.0 & & 7.5 & $86 \%$ & $\mathrm{mgh}$ & mgr. \\
\hline 4 & 20.2 & & 7.4 & $81 \%$ & Cond. & Hardness \\
\hline 5 & 21.5 & & 7.2 & $81 \%$ & Intitial & Initial \\
\hline 6 & 22.2 & & 7.3 & $84 \%$ & 285 & 11.4 \\
\hline 7 & $\overline{21.2}$ & & 7.5 & $84 \%$ & Final & Frnal \\
\hline 8 & 20.8 & & 7.1 & $78 \%$ & 43.0 & 3.8 \\
\hline 9 & 20.3 & & 8.1 & $89 \%$ & umhos $/ \mathrm{cm}$ & mgr. \\
\hline 10 & 21.0 & 6.98 & 8.1 & $91 \%$ & Aeration: & \\
\hline
\end{tabular}

\section{SURVIVAL AND GROWTH RESULTS}

CONTROL TREATMENT

\begin{tabular}{|c|c|c|c|c|c|c|}
\hline & \multicolumn{3}{|c|}{ \# Organisms } & \multirow[b]{2}{*}{$\begin{array}{c}\% \\
\text { Survival }\end{array}$} & \multirow{2}{*}{$\begin{array}{l}\text { Mean } \\
\text { Length } \\
(\mathrm{mm})\end{array}$} & \multirow{2}{*}{$\begin{array}{l}\text { Standard } \\
\text { Deviation }\end{array}$} \\
\hline & Initial & $\begin{array}{c}\text { Live at } \\
\text { End }\end{array}$ & $\begin{array}{c}\text { Dead } \\
\text { at End }\end{array}$ & & & \\
\hline A & 10 & 10 & 0 & $100 \%$ & & \\
\hline $\mathrm{B}$ & 10 & 6 & 4 & $60 \%$ & & \\
\hline $\mathrm{C}$ & 10 & 9 & 1 & $90 \%$ & & \\
\hline$\overline{\mathrm{D}}$ & 10 & 8 & 2 & $80 \%$ & & \\
\hline $\mathrm{E}$ & 10 & 10 & 0 & $100 \%$ & & \\
\hline F & 10 & 10 & 0 & $100 \%$ & & \\
\hline $\mathrm{G}$ & 10 & 5 & 5 & $50 \%$ & & \\
\hline $\mathrm{H}$ & 10 & 9 & 1 & $90 \%$ & & \\
\hline Mean & 10.0 & & & $84 \%$ & & \\
\hline
\end{tabular}

SAMPLE TREATMENT

\begin{tabular}{|c|c|c|c|c|c|c|}
\hline & \multicolumn{3}{|c|}{ \# Organisms } & \multirow[b]{2}{*}{$\begin{array}{c}\% \\
\text { Survival }\end{array}$} & \multirow{2}{*}{$\begin{array}{l}\text { Mean } \\
\text { Length } \\
\text { (mm) }\end{array}$} & \multirow{2}{*}{$\begin{array}{l}\text { Standard } \\
\text { Deviation }\end{array}$} \\
\hline & Initial & $\begin{array}{c}\text { Live at } \\
\text { End }\end{array}$ & $\begin{array}{l}\text { Dead } \\
\text { at End }\end{array}$ & & & \\
\hline $\mathrm{A}$ & 10 & 6 & 4 & $60 \%$ & & \\
\hline $\mathrm{B}$ & 10 & 7 & 3 & $70 \%$ & & \\
\hline C & 10 & 3 & 7 & $30 \%$ & & \\
\hline D & 10 & 5 & 5 & $50 \%$ & & \\
\hline$E$ & 10 & 3 & 7 & $30 \%$ & & \\
\hline$F$ & 10 & 3 & 7 & $30 \%$ & & \\
\hline $\mathrm{G}$ & 10 & 6 & 4 & $60 \%$ & & \\
\hline $\mathrm{H}$ & 10 & 6 & 4 & $60 \%$ & & \\
\hline Mean & 10.0 & & & $49 \%$ & & \\
\hline
\end{tabular}




\section{DAY AMPHIPOD SEDIMENT TOXICITY TEST}

\begin{tabular}{|l|l|l|}
\hline Lab D\# & Client: $\quad$ WSRC & Start Date: $8 / 4 / 99$ \\
\cline { 2 - 4 } T12313 & Sample Identification: B-8 & End Date: $8 / 14 / 99$ \\
\hline
\end{tabular}

\section{RESULTS}

\begin{tabular}{|l|r|}
\hline \multicolumn{2}{|c|}{$\begin{array}{c}\text { SURVIVAL DATA } \\
\text { Mean of Eight Replicates }\end{array}$} \\
\cline { 2 - 2 } & Mean \% \\
\hline Control & $83.8 \%$ \\
\hline Sample & $48.7 \%$ \\
\hline
\end{tabular}

\begin{tabular}{|l|l|}
\hline \multicolumn{2}{|c|}{$\begin{array}{c}\text { GROWTH DATA } \\
\text { Mean Length (mm) }\end{array}$} \\
\cline { 2 - 2 } & Mean \\
\hline Control & NA \\
\hline Sample & NA \\
\hline
\end{tabular}

\section{STATISTICAL ANALYSES}

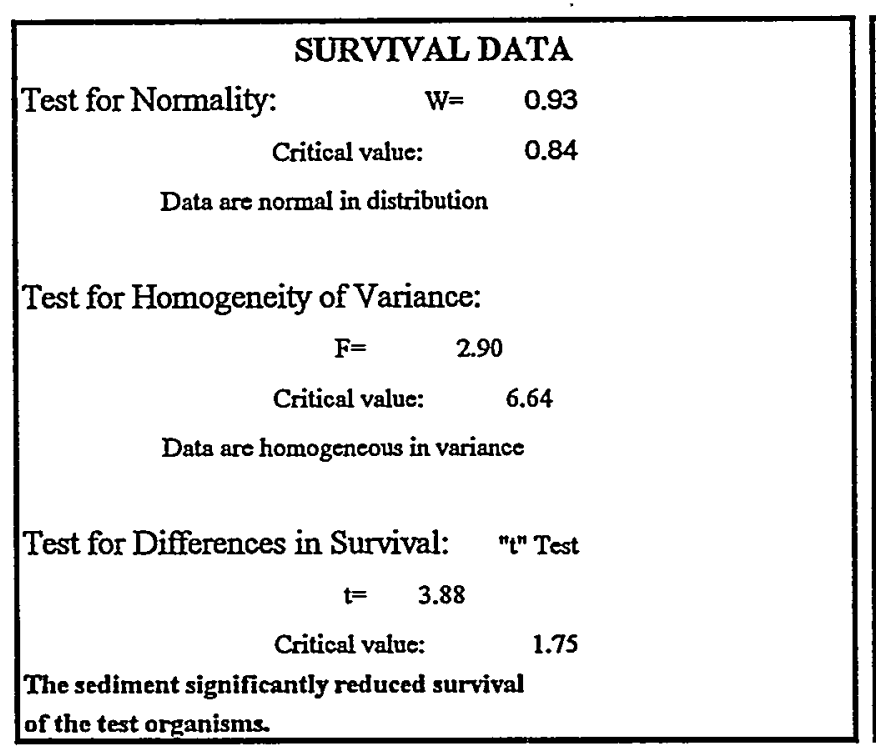

\begin{tabular}{|c|c|}
\hline \multicolumn{2}{|c|}{ GROWTH DATA } \\
\hline \multicolumn{2}{|l|}{ Test for Normality: $\quad W=N A$} \\
\hline Critical value: & 0.84 \\
\hline \multicolumn{2}{|l|}{ NA } \\
\hline \multicolumn{2}{|l|}{ Test for Homogeneity of Variance: } \\
\hline $\mathrm{F}=$ & NA \\
\hline Critical value: & 6.635 \\
\hline NA & \\
\hline \multicolumn{2}{|c|}{ Test for Differences in Survival: $\quad " t^{n}$ Test } \\
\hline$t=N A$ & \\
\hline Critical value: & 1.94 \\
\hline
\end{tabular}

\section{COMMENTS}

None

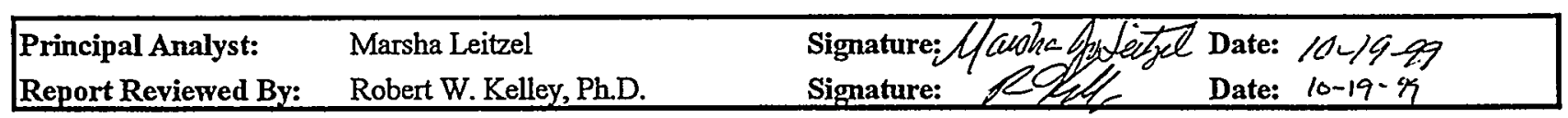

\section{CONCLUSIONS}

The sediment reduces survival of the test organisms. 
(864) 877-6942 - FAX (864) 877-6938

\section{DAY AMPHIPOD SEDIMENT TOXICITY TEST}

\section{EPA/600/R-94/024 Method 100.1}

Test Organism: Hyailela azteca

\section{Client: WSRC}

Sample-Identification: $\quad$ BGCH5

Test Start Date: $\quad 8 / 4 / 99$ 
WSRC-TR-99-00380

\section{DAY AMPHIPOD SEDIMENT TOXICITY TEST}

\begin{tabular}{|l|l|l|}
\hline Lab W\# & Client: $\quad$ WSRC & Start Date: $8 / 4 / 99$ \\
\cline { 2 - 4 } T12308 & Sample Identification: BGCH5 & End Date: $8 / 14 / 99$ \\
\hline
\end{tabular}

\section{Test System}

EPA/600/R-94/024 Method 100.1

The test was set as a Pass/Fail test with a control treatment and one sample treatment. Each treatment replicate consisted of $100 \mathrm{~g}$ of sediment and $175 \mathrm{~mL}$ of dilution water. There were eight replicates for each treatment. Ten test organisms were placed in each replicate. Sediment from a local stream was used in the control treatment. Test organisms were fed with YCT food. The test was conducted at a temperature of $23^{\circ} \mathrm{C}$ and a light cycle of $16 \mathrm{hr}$ light $/ 8 \mathrm{hr}$ dark. Test vessels were $500 \mathrm{~mL}$ plastic beakers placed in a constant temperature incubator room.

Order: Amphipoda

Species: Hyallela azteca

Source: Aquatic Biosystems, Fort Collins, CO

Life Stage: $\quad$ 7-14 days

$\%$ Mortality During $48 \mathrm{Hr}$ Prior to Test::

Taxonomic Verification: RWK 8/4/99

Culturing: as received

Observations: Cultures healthy

Acclimation: None
$0 \%$

\section{Control Sediment}

Source: Resurrection Creek

Collection Date: 8/4/99

Preservation: kept at $0-4^{\circ} \mathrm{C}$ in plastic

Date/Time Added to Test Chambers: $83 / 59$

Observations: silt and organic

Collection Method: grab

Disposal

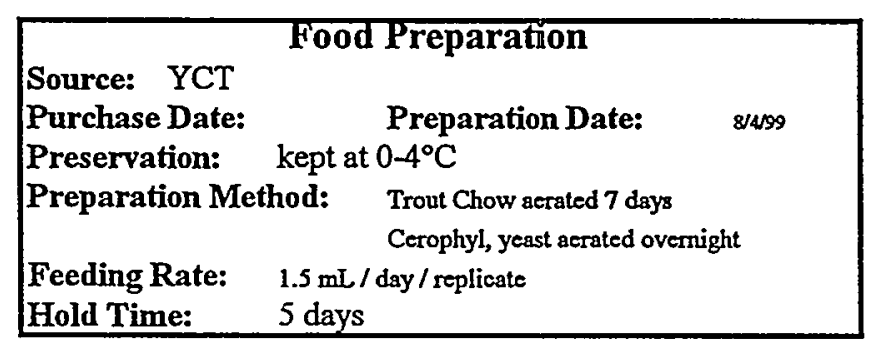

\section{Dilution Water}

Type: Surface Water

Collection Date 8/4/99

Alkalinity

Hardness

Conductivity

$p H$ :

Initial

10.4 .

11.4

285

7.63
$\mathrm{mg} / \mathbf{L}$
$m g / L$
umhovecm
units

Preparation Method:

Grab sample

BGCH5

Collection Date 7/19/99 Homogenized?: yes

Preservation: kept at $0-4^{\circ} \mathrm{C}$ in plastic

Date/Time Added to Test Chambers: $83 / 99$

Observations:

Collection Method: grab

Shipment: overnight

\begin{tabular}{|l|}
\hline \multicolumn{1}{c|}{ Test Chambers } \\
Type: Plastic $500 \mathrm{~mL}$ beakers \\
Sediment Volume: $\quad 100 \mathrm{~mL}$ \\
Sediment Depth $4 \mathrm{~cm}$ \\
Overlying Water Volume: $\quad 175 \mathrm{~mL}$ \\
Overlying Water Depth: $\quad 5 \mathrm{~cm}$ \\
Aeration: Not needed
\end{tabular}




\section{DAY AMPHIPOD SEDIMENT TOXICITY TEST}

\begin{tabular}{|l|l|l|}
\hline Lab D\# & Client: $\quad$ WSRC & Start Date: $8 / 4 / 99$ \\
\cline { 2 - 4 } T12308 & Sample Identification: BGCH5 & End Date: $8 / 14 / 99$ \\
\hline
\end{tabular}

\section{WATER CHEMISTRY TEST METHODS}

Temperature: SM 18th Ed. 2550

Dissolved Oxygen: SM 18th Ed. 4500-O

pH: SM 18th Ed. 4500-H+
Alkalinity: SM 18th Ed. 2320

Hardness: SM 18th Ed. 2340

Conductivity: SM 18th Ed. 2510

\section{WATER CHEMISTRY TEST DATA - OVERLYING WATER}

CONTROL TREATMENT

\begin{tabular}{|c|c|c|c|c|c|c|}
\hline & Temp. & $\mathrm{pH}$ & D.O. & D.O. & Alkalinity & Ammonia \\
\hline Test Day & C & units & $\mathrm{mg} / \mathrm{L}$ & $\%$ sat. & Initial & Initial \\
\hline Start & 22.3 & 7.63 & 8.0 & $92 \%$ & 10.4 & $<0.20$ \\
\hline 1 & 20.5 & & 7.9 & $87 \%$ & Final & Final \\
\hline 2 & 22.6 & & 7.6 & $87 \%$ & 88.2 & $<0.1$ \\
\hline 3 & 22.0 & & 7.5 & $86 \%$ & $\mathrm{mg} / \mathrm{L}$ & $\mathrm{mg} \Omega$ \\
\hline 4 & 20.2 & & 7.8 & $86 \%$ & Cond. & Hardness \\
\hline 5 & 21.5 & & 7.0 & $79 \%$ & Initial & Initial \\
\hline 6 & 22.2 & & 7.2 & $83 \%$ & 285 & 11.4 \\
\hline 7 & 21.2 & & 6.8 & $76 \%$ & Final & Final \\
\hline 8 & 20.8 & & 6.8 & $75 \%$ & 175.0 & 49.4 \\
\hline 9 & 20.2 & & 8.0 & $88 \%$ & umhos/cm & $m g h$ \\
\hline 10 & 21.0 & 7.46 & 4.9 & $55 \%$ & Aeration: & \\
\hline
\end{tabular}

\section{SURVIVAL AND GROWTH RESULTS}

CONTROL TREATMENT

\begin{tabular}{|c|c|c|c|c|c|c|}
\hline & \multicolumn{3}{|c|}{ \# Organisms } & \multirow[b]{2}{*}{$\begin{array}{c}\% \\
\text { Survival }\end{array}$} & \multirow{2}{*}{$\begin{array}{l}\text { Mean } \\
\text { Length } \\
\text { (mm) }\end{array}$} & \multirow{2}{*}{$\begin{array}{l}\text { Standard } \\
\text { Deviation }\end{array}$} \\
\hline & Initial & $\begin{array}{l}\text { Live at } \\
\text { End }\end{array}$ & $\begin{array}{l}\text { Dead } \\
\text { at End }\end{array}$ & & & \\
\hline $\mathrm{A}$ & 10 & 10 & 0 & $100 \%$ & & \\
\hline B & 10 & 6 & 4 & $60 \%$ & & \\
\hline C & 10 & 9 & 1 & $90 \%$ & & \\
\hline $\mathrm{D}$ & 10 & 8 & 2 & $80 \%$ & & \\
\hline$\overline{\mathrm{E}}$ & 10 & 10 & 0 & $100 \%$ & & \\
\hline $\bar{F}$ & 10 & 10 & 0 & $100 \%$ & & \\
\hline $\mathrm{G}$ & 10 & 5 & 5 & $50 \%$ & & \\
\hline $\mathrm{H}$ & 10 & $\overline{9}$ & 1 & $90 \%$ & & \\
\hline Mean & 10.0 & & & $84 \%$ & & \\
\hline
\end{tabular}

SAMPLE TREATMENT

\begin{tabular}{|c|c|c|c|c|c|c|}
\hline & Temp. & $\mathrm{pH}$ & D.O. & D.O. & Alkalinity & Ammonia \\
\hline Test Day & C & units & $\mathrm{mg} / \mathrm{L}$ & $\%$ sat. & Initisl & Initial \\
\hline Start & 22.3 & 7.63 & 8.0 & $92 \%$ & 10.4 & $<0.20$ \\
\hline 1 & 20.3 & & 8.1 & $89 \%$ & Final & Fmal \\
\hline 2 & 22.6 & & 7.8 & $.90 \%$ & 6.1 & $<0.1$ \\
\hline 3 & 22.2 & & 7.6 & $87 \%$ & $\mathrm{mgh}$ & mgh \\
\hline 4 & 20.2 & & 7.4 & $81 \%$ & Cond. & Hardness \\
\hline 5 & 21.5 & & 7.1 & $80 \%$ & Initial & Initial \\
\hline 6 & 22.2 & & 7.4 & $85 \%$ & 285 & 11.4 \\
\hline 7 & 21.2 & & 7.2 & $81 \%$ & Final & Final \\
\hline 8 & 20.8 & & 7.1 & $78 \%$ & 3.8 & 43.0 \\
\hline 9 & 22.0 & & 7.8 & $90 \%$ & umhosicm & $\mathrm{mgh}$ \\
\hline 10 & 21.0 & 7.49 & 8.1 & $91 \%$ & \multicolumn{2}{|c|}{ Acration: none } \\
\hline
\end{tabular}

SAMPLE TREATMENT

\begin{tabular}{|c|c|c|c|c|c|c|}
\hline & \multicolumn{3}{|c|}{ \# Organisms } & \multirow[b]{2}{*}{$\begin{array}{c}\% \\
\text { Survival } \\
\end{array}$} & \multirow{2}{*}{$\begin{array}{l}\text { Mean } \\
\text { Length } \\
\text { (mm) }\end{array}$} & \multirow{2}{*}{$\begin{array}{l}\text { Standard } \\
\text { Deviation }\end{array}$} \\
\hline & Initial & $\begin{array}{c}\text { Live at } \\
\text { End }\end{array}$ & $\begin{array}{l}\text { Dead } \\
\text { at End }\end{array}$ & & & \\
\hline A & 10 & 1 & 9 & $10 \%$ & & \\
\hline $\mathrm{B}$ & 10 & 4 & 6 & $40 \%$ & & \\
\hline $\mathrm{C}$ & 10 & 3 & 7 & $30 \%$ & & \\
\hline $\mathrm{D}$ & 10 & 3 & 7 & $30 \%$ & & \\
\hline$E$ & 10 & 8 & 2 & $80 \%$ & & \\
\hline $\mathbf{F}$ & 10 & 4 & 6 & $40 \%$ & & \\
\hline $\mathbf{G}$ & 10 & 9 & 1 & $90 \%$ & & \\
\hline $\mathrm{H}$ & 10 & 6 & 4 & $60 \%$ & & \\
\hline Mean & 10.0 & & & $48 \%$ & & \\
\hline
\end{tabular}




\section{DAY AMPHIPOD SEDIMENT TOXICITY TEST}

\begin{tabular}{|l|l|l|}
\hline Lab W\# & Client: WSRC $\quad$ Start Date: 8/4/99 \\
\cline { 2 - 3 } T12308 Sample Identification: BGCH5 & End Date: 8/14/99 \\
\hline
\end{tabular}

\section{RESULTS}

\begin{tabular}{|l|c|}
\hline \multicolumn{2}{|c|}{$\begin{array}{c}\text { SURVIVAL DATA } \\
\text { Mean of Eight Replicates }\end{array}$} \\
\cline { 2 - 2 } & Mean \% \\
\hline Control & $83.8 \%$ \\
\hline Sample & $47.5 \%$ \\
\hline
\end{tabular}

\begin{tabular}{|l|c|}
\hline \multicolumn{2}{|c|}{$\begin{array}{c}\text { GROWTH DATA } \\
\text { Mean Length (mm) }\end{array}$} \\
\cline { 2 - 2 } & Mean \\
\hline Control & NA \\
\hline Sample & NA \\
\hline
\end{tabular}

\section{STATISTICAL ANALYSES}

\begin{tabular}{|ccc|}
\hline \multicolumn{3}{|c}{ SURVIVAL DATA } \\
Test for Normality: $\quad$ W= & 0.95 \\
& Critical value: & 0.84 \\
Data are normal in distribution &
\end{tabular}

Test for Homogeneity of Variance:

$$
\begin{array}{cl}
\mathrm{F}= & \multicolumn{1}{c}{1.12} \\
\text { Critical value: } & 6.64
\end{array}
$$

Data are homogeneous in variance

Test for Differences in Survival: " "t" Test

$$
\begin{array}{cc}
t=3.16 \\
\text { Critical value: }
\end{array}
$$

The sediment significantly reduced survival of the test organisms.

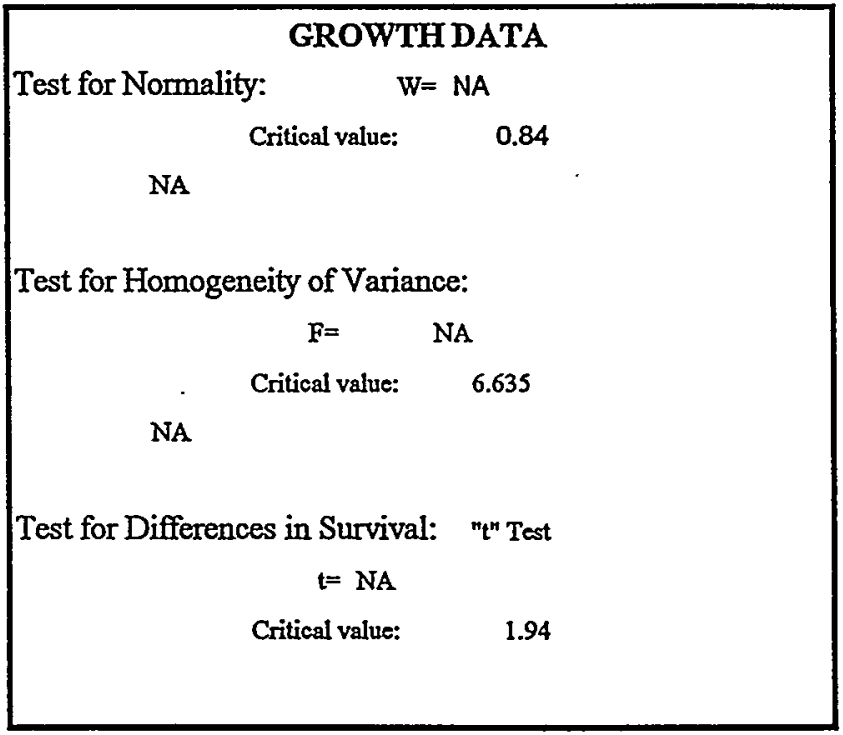

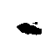

\section{COMMENTS}

None

\begin{tabular}{lll}
\hline Principal Analyst: & Marsha Leitzel & Signature: Mlaskaidetetyel Date: $104 / 9-95$ \\
Report Reviewed By: & Robert W. Kelley, Ph.D. & Signature:
\end{tabular}

\section{CONCLUSIONS}

The sediment reduces survival of the test organisms. 


\title{
10 DAY AMPHIPOD SEDIMENT TOXICITY TEST
}

\author{
EPA/600/R-94/024 Method 100.1 \\ Test Organism: Hyallela azteca
}

\author{
Client: WSRC \\ Sample Identification: $\quad$ BGCH7
}

Test Start Date: $\quad 8 / 4 / 99$ 


\section{DAY AMPHIPOD SEDIMENT TOXICITY TEST}

\begin{tabular}{|l|l|l|}
\hline Lab ID\# & Client: $\quad$ WSRC & Start Date: 8/4/99 \\
\cline { 2 - 4 } T12307 & Sample Identification: BGCH7 & End Date: $8 / 14 / 99$ \\
\hline
\end{tabular}

\section{Test System \\ EPA/600/R-94/024 Method 100.1}

The test was set as a Pass/Fail test with a control treatment and one sample treatment. Each treatment replicate consisted of $100 \mathrm{~g}$ of sediment and $175 \mathrm{~mL}$ of dilution water. There were eight replicates for each treatment. Ten test organisms were placed in each replicate. Sediment from a local stream was used in the control treatment. Test organisms were fed with YCT food. The test was conducted at a temperature of $23^{\circ} \mathrm{C}$ and a light cycle of $16 \mathrm{hr}$ light/8 hr dark. Test vessels were $500 \mathrm{~mL}$ plastic beakers placed in a constant temperature incubator room.

\author{
Order: Amphipoda \\ Species: Hyallela azteca \\ Source: Aquatic Biosystems, Fort Collins, CO \\ Life Stage: 7 to 14 days \\ $\%$ Mortality During 48 Hr Prior to Test:: \\ Taxonomic Verification: RWK 8/4/99 \\ Culturing: as received \\ Observations: Cultures healthy \\ Acclimation: None
}

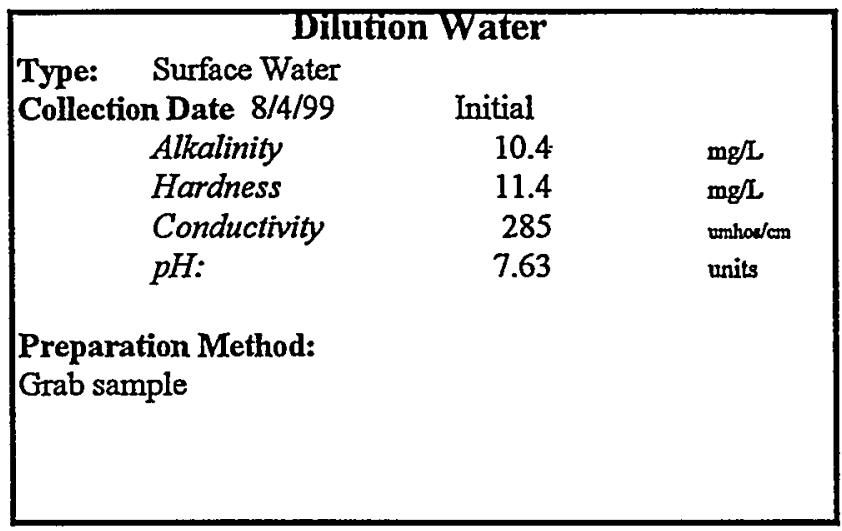

Control Sediment
Source: Resurrection Creek
Collection Date: $8 / 4 / 99$
Preservation: kept at $0-4^{\circ} \mathrm{C}$ in plastic
Date/Time Added to Test Chambers: $8 / 3 / 99$
Observations: silt and organic
Collection Method: grab
Disposal

\begin{tabular}{l} 
Test Sediment \\
Collection Date $7 / 19 / 99 \quad$ Homogenized?: yes \\
Preservation: kept at $0-4^{\circ} \mathrm{C}$ in plastic \\
Date/Time Added to Test Chambers: \&/999 \\
Observations: \\
Collection Method: grab \\
Shipment: overnight \\
\hline
\end{tabular}

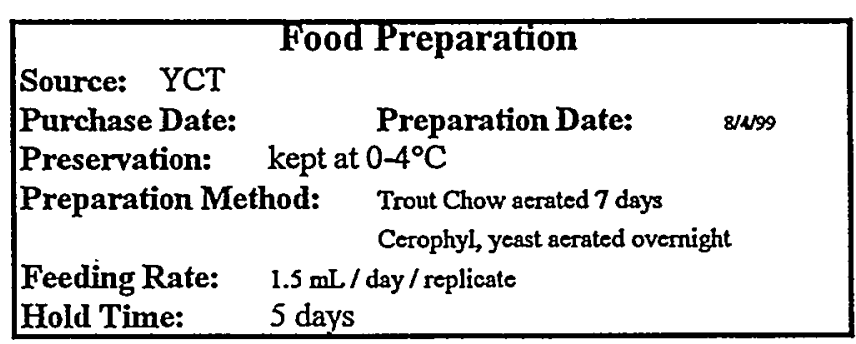




\section{DAY AMPHIPOD SEDIMENT TOXICITY TEST}

\begin{tabular}{|l|l|l|}
\hline Lab W\#\# & Client: $\quad$ WSRC & Start Date: $8 / 4 / 99$ \\
\cline { 2 - 4 } T12307 & Sample Identification: BGCH7 & End Date: $8 / 14 / 99$ \\
\hline
\end{tabular}

WATER CHEMISTRY TEST METHODS

Temperature: SM 18th Ed. 2550

Dissolved Oxygen: SM 18th Ed. 4500-O

pH: SM 18th Ed. 4500-H+

\author{
Alkalinity: SM 18th Ed. 2320 \\ Hardness: SM 18th Ed. 2340 \\ Conductivity: SM 18th Ed. 2510
}

WATER CHEMISTRY TEST DATA - OVERLYING WATER

CONTROL TREATMENT

\begin{tabular}{|c|c|c|c|c|c|c|}
\hline & Temp. & $\mathrm{pH}$ & D.o. & D.O. & Alkalinity & Ammonia \\
\hline Test Day & $\mathrm{C}$ & units & $\mathrm{mg} / \mathrm{L}$ & $\%$ sat. & Initial & Initial \\
\hline Start & 22.3 & 7.63 & 8.0 & $92 \%$ & 10.4 & $<0.20$ \\
\hline$\overline{1}$ & 20.5 & & 7.9 & $87 \%$ & Final & Frial \\
\hline 2 & 22.6 & & 7.6 & $87 \%$ & 88.2 & $<0.1$ \\
\hline 3 & 22.0 & & 7.5 & $86 \%$ & $\mathrm{mgh}$ & $\mathrm{mgh}$ \\
\hline$\overline{4}$ & 20.2 & & 7.8 & $86 \%$ & Cond. & Hardness \\
\hline 5 & 21.5 & & 7.0 & $79 \%$ & Intital & Intitial \\
\hline$\overline{6}$ & 22.2 & & 7.2 & $83 \%$ & 285 & 11.4 \\
\hline 7 & 21.2 & & 6.8 & $76 \%$ & Final & Final \\
\hline 8 & 20.8 & & 6.8 & $75 \%$ & 175.0 & 49.4 \\
\hline 9 & 20.2 & & 8.0 & $88 \%$ & umhos/cm & man \\
\hline 10 & 21.0 & 7.46 & 4.9 & $55 \%$ & Aeration: & \\
\hline
\end{tabular}

SAMPLE TREATMENT

\begin{tabular}{|c|c|c|c|c|c|c|}
\hline & Temp. & $\mathrm{pH}$ & D.O. & D.O. & Alkalinity & Ammonia \\
\hline Test Day & C & units & $\mathrm{mg} / \mathrm{L}$ & $\%$ sat & Initial & Initial \\
\hline Start & 22.3 & 7.63 & 8.0 & $92 \%$ & 10.4 . & $<0.20$ \\
\hline 1 & 20.2 & & 8.0 & $88 \%$ & Frinal & Final \\
\hline 2 & 22.6 & & 7.2 & $.83 \%$ & 21.0 & $<0.1$ \\
\hline 3 & 21.1 & & 7.5 & $84 \%$ & mgr & $\mathrm{mgn}$ \\
\hline 4 & 20.2 & & 7.2 & $79 \%$ & Cond. & Hardness \\
\hline 5 & 21.5 & & 7.2 & $81 \%$ & Initial & Initiol \\
\hline 6 & 22.2 & & 7.5 & $86 \%$ & 285 & 11.4 \\
\hline 7 & 21.2 & & 7.1 & $80 \%$ & Final & Firal \\
\hline 8 & 20.8 & & 7.0 & $77 \%$ & 39.0 & 3.8 \\
\hline 9 & 19.7 & & 7.8 & $84 \%$ & umhos/cm & mgr \\
\hline 10 & 21.0 & 7.97 & 7.3 & $82 \%$ & Aeration & \\
\hline
\end{tabular}

\section{SURVIVAL AND GROWTH RESULTS}

CONTROL TREATMENT

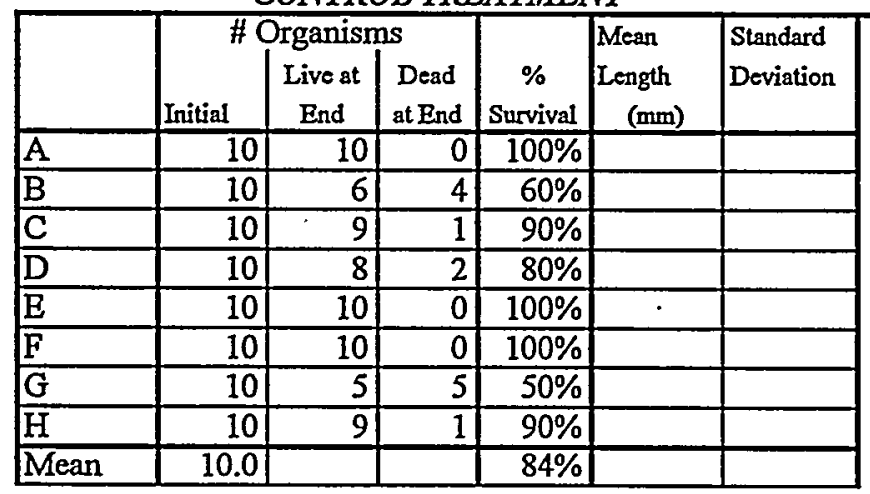

SAMPLE TREATMENT

\begin{tabular}{|c|c|c|c|c|c|c|}
\hline & \multicolumn{3}{|c|}{ \# Organisms } & \multirow[b]{2}{*}{$\begin{array}{c}\% \\
\text { Survival }\end{array}$} & \multirow{2}{*}{$\begin{array}{l}\text { Mean } \\
\text { Length } \\
(\mathrm{mm})\end{array}$} & \multirow{2}{*}{$\begin{array}{l}\text { Standard } \\
\text { Deviation }\end{array}$} \\
\hline & Initial & $\begin{array}{l}\text { Live at } \\
\text { End }\end{array}$ & $\begin{array}{l}\text { Dead } \\
\text { at End }\end{array}$ & & & \\
\hline$A$ & 10 & 1 & 9 & $10 \%$ & & \\
\hline$B$ & 10 & 1 & 9 & $10 \%$ & & \\
\hline C & 10 & 4 & 6 & $40 \%$ & & \\
\hline $\mathrm{D}$ & 10 & 6 & 4 & $60 \%$ & & \\
\hline$E$ & 10 & 4 & 6 & $40 \%$ & & \\
\hline $\mathrm{F}$ & 10 & 1 & 9 & $10 \%$ & & \\
\hline $\mathbf{G}$ & 10 & 1 & 9 & $10 \%$ & & \\
\hline $\mathrm{H}$ & 10 & .7 & 3 & $70 \%$ & & \\
\hline Mean & 10.0 & & & $31 \%$ & & \\
\hline
\end{tabular}


10 DAY AMPHIPOD SEDIMENT TOXICITY TEST

\begin{tabular}{|l|l|l|}
\hline Lab D\# & Client: $\quad$ WSRC & Start Date: $8 / 4 / 99$ \\
\hline T12307 & Sample Identification: BGCH7 & End Date: $8 / 14 / 99$ \\
\hline
\end{tabular}

\section{RESULTS}

\begin{tabular}{|l|r|}
\hline \multicolumn{2}{|c|}{$\begin{array}{c}\text { SURVIVAL DATA } \\
\text { Mean of Eight Replicates }\end{array}$} \\
\cline { 2 - 2 } & Mean \% \\
\hline Control & $83.8 \%$ \\
\hline Sample & $31.3 \%$ \\
\hline
\end{tabular}

\begin{tabular}{|l|l|}
\hline \multicolumn{2}{|c|}{$\begin{array}{l}\text { GROWTH DATA } \\
\text { Mean Length (mm) }\end{array}$} \\
\cline { 2 - 2 } & Mean \\
\hline Control & NA \\
\hline Sample & NA \\
\hline
\end{tabular}

\section{STATISTICAL ANALYSES}

\begin{tabular}{|c|c|}
\hline \multicolumn{2}{|c|}{ SURVIVAL DATA } \\
\hline Test for Normality: & 0.93 \\
\hline Critical value: & 0.84 \\
\hline \multicolumn{2}{|l|}{ Data are normal in distribution } \\
\hline \multicolumn{2}{|l|}{ Test for Homogeneity of Variance: } \\
\hline $\mathrm{F}=$ & 1.05 \\
\hline Critical value: & 6.64 \\
\hline \multicolumn{2}{|c|}{ Data are homogeneous in variance } \\
\hline \multicolumn{2}{|c|}{ Test for Differences in Survival: $\quad{ }^{n} \mathrm{t}$ Test } \\
\hline$t=, \quad 4.66$ & \\
\hline Critical value: & 1.75 \\
\hline $\begin{array}{l}\text { The sediment significantly reduced sur } \\
\text { of the test organisms. }\end{array}$ & \\
\hline
\end{tabular}

\begin{tabular}{|c|c|}
\hline GROWTH & IDATA \\
\hline Test for Normality: & NA \\
\hline Critical value: & $0: 84$ \\
\hline NA & \\
\hline Test for Homogeneity of Variano & \\
\hline$F=$ & NA \\
\hline Critical value: & 6.635 \\
\hline NA & \\
\hline $\begin{array}{r}\text { Test for Differences in Survival: } \\
\qquad t=\mathrm{NA}\end{array}$ & ${ }^{n} \mathrm{t} n$ Test \\
\hline Critical value: & 1.94 \\
\hline
\end{tabular}

\section{COMMENTS}

None

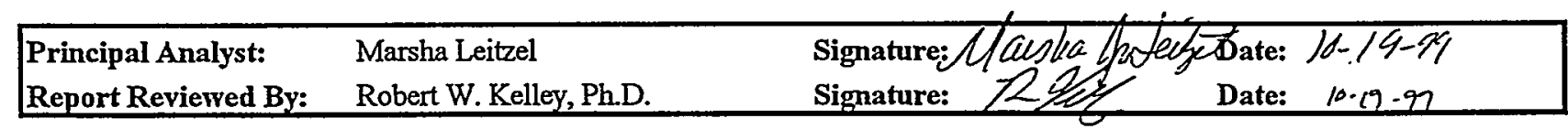

\section{CONCLUSIONS}

The sediment reduces survival of the test organisms. 


\section{DAY AMPHIPOD SEDIMENT TOXICITY TEST}

EPA/600/R-94/024 Method 100.1

Test Organism: Hyallela azteca

$\begin{aligned} \text { Client: } & \text { WSRC } \\ \text { Sample Identification: } & \text { C-10 }\end{aligned}$

Test Start Date: $\quad 8 / 4 / 99$ 


\section{DAY AMPHIPOD SEDIMENT TOXICITY TEST}

\begin{tabular}{|c|c|c|c|}
\hline \multirow{2}{*}{\begin{tabular}{|l|} 
Lab ID\# \\
$\mathrm{T} 12311$ \\
\end{tabular}} & WSRC & Start Date: & $8 / 4 / 99$ \\
\hline & Sample Identification: $\quad$ C-10 & End Date: & $8 / 14 / 99$ \\
\hline
\end{tabular}

\section{Test System}

EPA/600/R-94/024 Method 100.1

The test was set as a Pass/Fail test with a control treatment and one sample treatment. Each treatment replicate consisted of $100 \mathrm{~g}$ of sediment and $175 \mathrm{~mL}$ of dilution water. There were eight replicates for each treatment. Ten test organisms were placed in each replicate. Sediment from a local stream was used in the control treatment. Test organisms were fed with YCT food. The test was conducted at a temperature of $23^{\circ} \mathrm{C}$ and a light cycle of $16 \mathrm{hr}$ light/8 hr dark. Test vessels were $500 \mathrm{~mL}$ plastic beakers placed in a constant temperature incubator room.

Order: Amphipoda

\section{Test Organisms}

Species: Hyallela azteca

Source: Aquatic Biosystems, Fort Collins, CO

Life Stage: $\quad 7$ to 14 days

\% Mortality During $48 \mathrm{Hr}$ Prior to Test:: $\quad 0 \%$

Taxonomic Verification: RWK 8/4/99

Culturing: as received

Observations: Cultures healthy

Acclimation: None

\section{Dilution Water}

Type: Surface Water

Collection Date 8/4/99

$$
\text { Alkalinity }
$$

Hardness

Conductivity

$p H$ :

Initial

10.4

11.4

$285^{\circ}$

7.63

mgll

umhou/crn

units

Preparation Method:

Grab sample

\section{Test Sediment}

\section{Source: C-10}

Collection Date 7/16/99 Homogenized?: yes

Preservation: kept at $0-4^{\circ} \mathrm{C}$ in plastic

Date/Time Added to Test Chambers: $8 / 399$

Observations:

Collection Method: grab

Shipment: overnight
Collection Method: grab

Disposal

\begin{tabular}{|c|c|}
\hline \multicolumn{2}{|c|}{ Food Preparation } \\
\hline $\begin{array}{l}\text { Source: YCT } \\
\text { Purchase Date: } \\
\text { Preservation: kept a }\end{array}$ & $\begin{array}{l}\text { Preparation Date: } \\
0-4^{\circ} \mathrm{C}\end{array}$ \\
\hline Preparation Method: & $\begin{array}{l}\text { Trout Chow aerated } 7 \text { days } \\
\text { Cerophyl yeast acrated overnight }\end{array}$ \\
\hline $\begin{array}{l}\text { Feeding Rate: } \\
\text { Hold Time: }\end{array}$ & day / replicate \\
\hline
\end{tabular}

\section{Test Chambers}

Type: Plastic $500 \mathrm{~mL}$ beakers

Sediment Volume: $\quad 100 \mathrm{~mL}$

Sediment Dept $4 \mathrm{~cm}$

Overlying Water Volume: $\quad 175 \mathrm{~mL}$

Overlying Water Depth: $\quad 5 \mathrm{~cm}$

Aeration: Not needed 


\section{DAY AMPHIPOD SEDIMENT TOXICITY TEST}

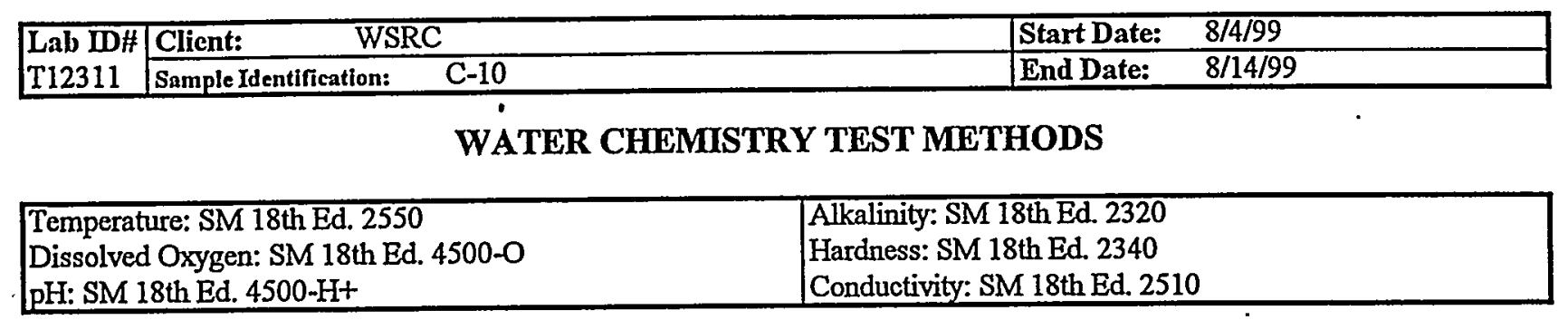

\section{WATER CHEMISTRY TEST DATA - OVERLYING WATER}

CONTROL TREATMENT

\begin{tabular}{|c|c|c|c|c|c|c|}
\hline & Temp. & $\mathrm{pH}$ & D.O. & D.O. & Alkalinity & Ammonia \\
\hline Test Day & C & units & $\mathrm{mg} / \mathrm{L}$ & $\%$ sat. & Initial & Initial \\
\hline Start & 22.3 & 7.63 & 8.0 & $92 \%$ & 10.4 & $<0.20$ \\
\hline 1 & 20.5 & & 7.9 & $87 \%$ & Final & Find \\
\hline 2 & 22.6 & & 7.6 & $87 \%$ & 88.2 & $<0.1$ \\
\hline 3 & 22.0 & & 7.5 & $86 \%$ & $\mathrm{mgh}$ & $m g h$ \\
\hline 4 & 20.2 & & 7.8 & $86 \%$ & Cond. & Hardness \\
\hline 5 & 21.5 & & 7.0 & $79 \%$ & Initial & Initial \\
\hline 6 & 22.2 & & 7.2 & $83 \%$ & 285 & 11.4 \\
\hline 7 & 21.2 & & 6.8 & $76 \%$ & Final & Final \\
\hline 8 & 20.8 & & 6.8 & $75 \%$ & 175.0 & 49.4 \\
\hline 9 & 20.2 & & 8.0 & $88 \%$ & umhosien & $\mathrm{mgh}$ \\
\hline 10 & 21.0 & 7.46 & 4.9 & $55 \%$ & Aeration: $\mathrm{n}$ & \\
\hline
\end{tabular}

SAMPLE TREATMENT

\begin{tabular}{|c|c|c|c|c|c|c|}
\hline & Temp. & $\mathrm{pH}$ & D.O. & D.O. & Alkalinity & Ammonia \\
\hline Test Day & C & units & $\mathrm{mg} / \mathrm{L}$ & \% sat. & Inital & Initial \\
\hline Start & 22.3 & 7.63 & 8.0 & $92 \%$ & 10.4 & $<0.20$ \\
\hline 1 & 20.3 & & 7.6 & $84 \%$ & Fral & Final \\
\hline 2 & 22.6 & & 8.0 & $92 \%$ & .10 .1 & $<0.1$ \\
\hline 3 & 21.0 & & 7.1 & $80 \%$ & $\mathrm{mgl}$ & $\mathrm{mgh}$ \\
\hline 4 & 20.2 & & 7.4 & $81 \%$ & Cond. & Hardiness \\
\hline 5 & 21.5 & & 7.2 & $81 \%$ & Initial & Initial \\
\hline 6 & 22.2 & & 7.5 & $86 \%$ & 285 & 11.4 \\
\hline 7 & 21.2 & & 7.8 & $88 \%$ & Final & Final \\
\hline 8 & 20.8 & & 7.6 & $84 \%$ & 43.0 & 5.7 \\
\hline 9 & 20.1 & & 8.1 & $89 \%$ & umhos/cm & $\mathrm{mg} \Omega$ \\
\hline 10 & 21.0 & 7.71 & 8.0 & $90 \%$ & Aeration: & \\
\hline
\end{tabular}

\section{SURVIVAL AND GROWTH RESULTS}

CONTROL TREATMENT

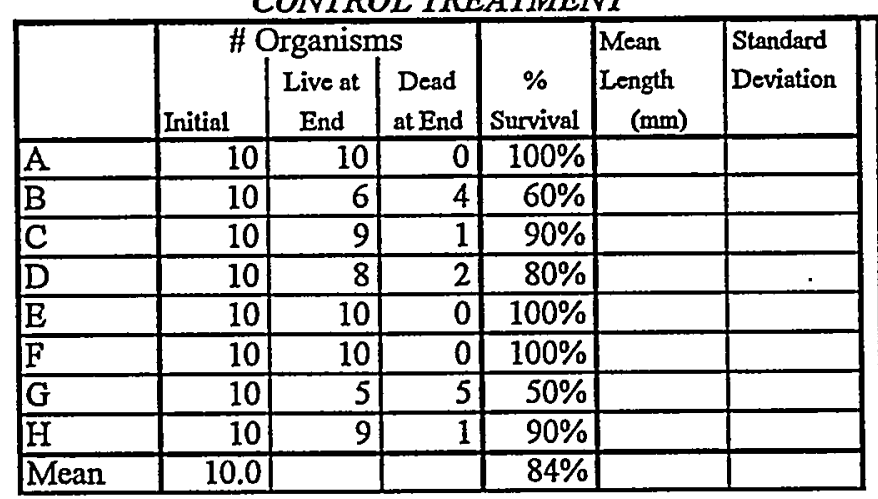

SAMPLE TREATMENT

\begin{tabular}{|c|c|c|c|c|c|c|}
\hline & \multicolumn{3}{|c|}{ \# Organisms } & \multirow[b]{2}{*}{$\begin{array}{c}\% \\
\text { Survival }\end{array}$} & \multirow{2}{*}{$\begin{array}{l}\text { Mean } \\
\text { Length } \\
(\mathrm{mm})\end{array}$} & \multirow{2}{*}{$\begin{array}{l}\text { Standard } \\
\text { Deviation }\end{array}$} \\
\hline & Initial & $\begin{array}{c}\text { Live at } \\
\text { End }\end{array}$ & $\begin{array}{l}\text { Dead } \\
\text { at End }\end{array}$ & & & \\
\hline A & 10 & 7 & 3 & $70 \%$ & & \\
\hline$\overline{\mathrm{B}}$ & 10 & 7 & 3 & $70 \%$ & & \\
\hline C & 10 & 8 & 2 & $80 \%$ & & \\
\hline $\mathrm{D}$ & 10 & 6 & 4 & $60 \%$ & & \\
\hline $\mathrm{E}$ & 10 & 8 & 2 & $80 \%$ & & \\
\hline $\mathrm{F}$ & 10 & 5 & 5 & $50 \%$ & $=$ & \\
\hline$G$ & 10 & 5 & 5 & $50 \%$ & & \\
\hline $\mathrm{H}$ & 10 & 5 & 5 & $50 \%$ & & \\
\hline Mean & 10.0 & & & $64 \%$ & & \\
\hline
\end{tabular}




\section{DAY AMPHIPOD SEDIMENT TOXICITY TEST}

\begin{tabular}{|l|l|l|}
\hline Lab ID\# & Client: $\quad$ WSRC & Start Date: $8 / 4 / 99$ \\
\cline { 2 - 4 } T12311 & Sample Identification: C-10 & End Date: $8 / 14 / 99$ \\
\hline
\end{tabular}

\section{RESULTS}

\begin{tabular}{|l|r|l|l|}
\hline \multicolumn{2}{|c|}{$\begin{array}{c}\text { SURVTVAL DATA } \\
\text { Mean of Eight Replicates }\end{array}$} & \multicolumn{3}{c|}{$\begin{array}{c}\text { GROWTH DATA } \\
\text { Mean Length (mm) }\end{array}$} \\
\cline { 2 - 3 } & & Mean \% \\
\cline { 2 - 3 } & $83.8 \%$ \\
\hline Control & $63.8 \%$ \\
\hline Sample & Control & Mean \\
\hline
\end{tabular}

\section{STATISTICAL ANALYSES}

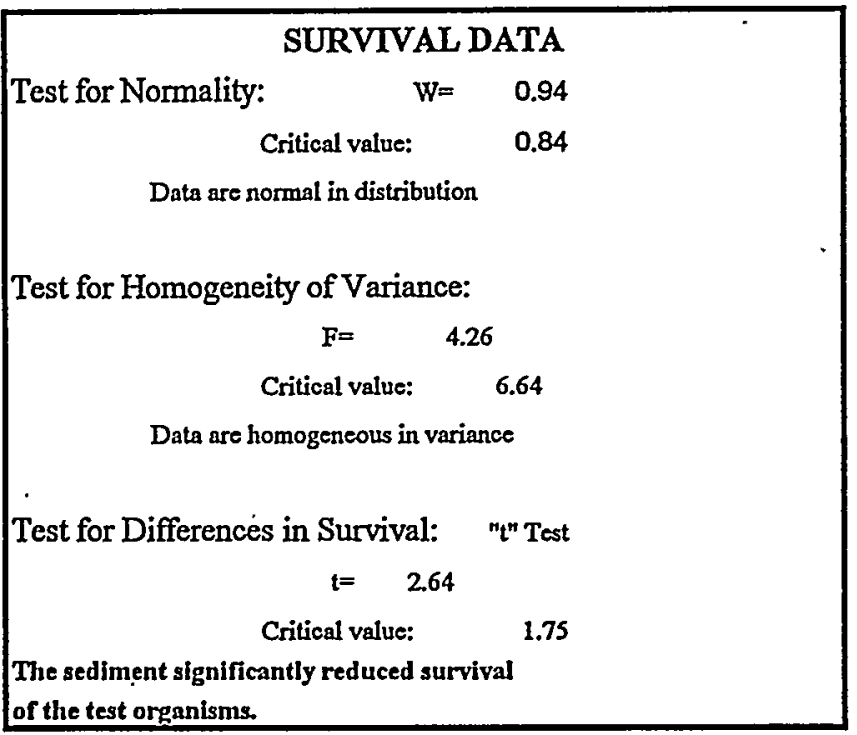

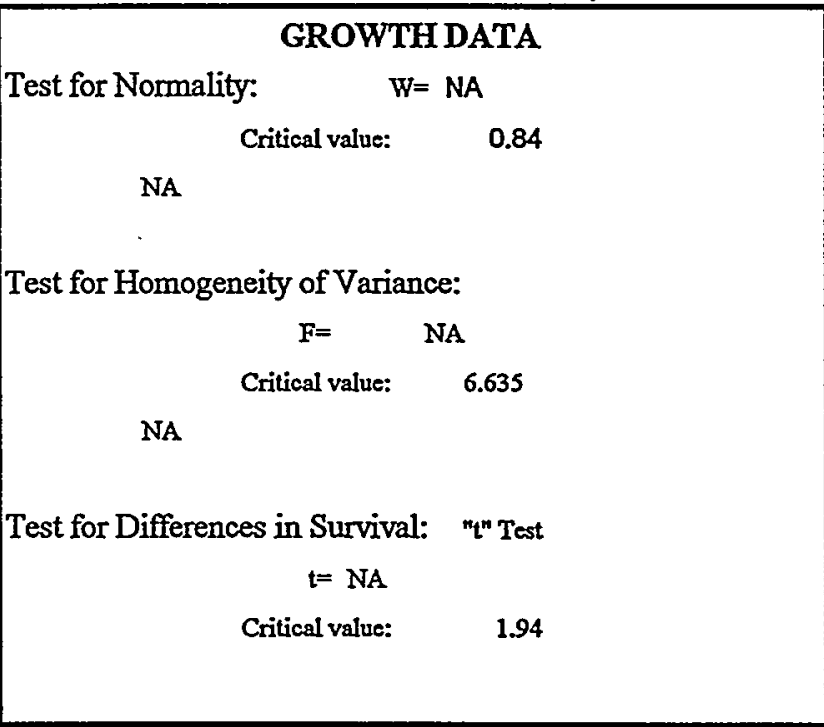

\section{COMMENTS}

The sediment reduces the survival of the test organisms.

\begin{tabular}{|c|c|c|}
\hline $\begin{array}{l}\text { Principal Analyst: } \\
\text { Report Reviewed By: }\end{array}$ & $\begin{array}{l}\text { Marsha Leitzel } \\
\text { Robert W. Kelley, Ph.D. }\end{array}$ & $\begin{array}{l}\text { Signature: Mashatesoleiget Date: } 10-19-99 \\
\text { Signature: } \\
\text { Date: 10-19-99 }\end{array}$ \\
\hline
\end{tabular}

\section{CONCLUSIONS}

The sediment does not adversely affect the survival and but does adversely effect the growth of freshwater amphipods. 
WSRC-TR-99-00380

(864) 877-6942 - FAX (864) 877-6938

\section{Day Chronic Definitive Sediment Toxicity Test}

Method: EPA/600/R-94/024 100.1

Test Organism: Hyallela azteca

Facility: WSRC

Sample ID: D-7

$$
\begin{aligned}
& \text { County: Aiken } \\
& \text { NPDESH: } \\
& \text { ETT\#: T12304 }
\end{aligned}
$$

Sample ID: August 4, 1999 


\section{CHRONIC DEFINITIVE SEDIMENT 10 DAY SURVIVAL TEST}

\begin{tabular}{|l|l|l|l|}
\hline Lab IDH & Client: & \multicolumn{1}{|c|}{ WSRC } & Start Date: \\
T12304 & Sample Identincation: $\quad$ D-7 & End Date: & $8 / 1499$ \\
\hline
\end{tabular}

\section{Test System}

\section{EPA/600/R-94/024 Method 100.1}

The test was set as a Definitive test with a control treatment and four sample treatments. Each treatment replicate consisted of $100 \mathrm{~g}$ of sediment and $175 \mathrm{~mL}$ of dilution water. There were four replicates for each treatment. Ten test organisms were placed in each replicate. Sediment from a local stream was used in the control treatment. Test organisms were fed with YCT food. The test was conducted at a temperature of $23^{\circ} \mathrm{C}$ and a light cycle of $16 \mathrm{hr}$ light $/ 8 \mathrm{hr}$ dark. Test vessels were $500 \mathrm{~mL}$ plastic beakers placed in a constant temperature incubator room.

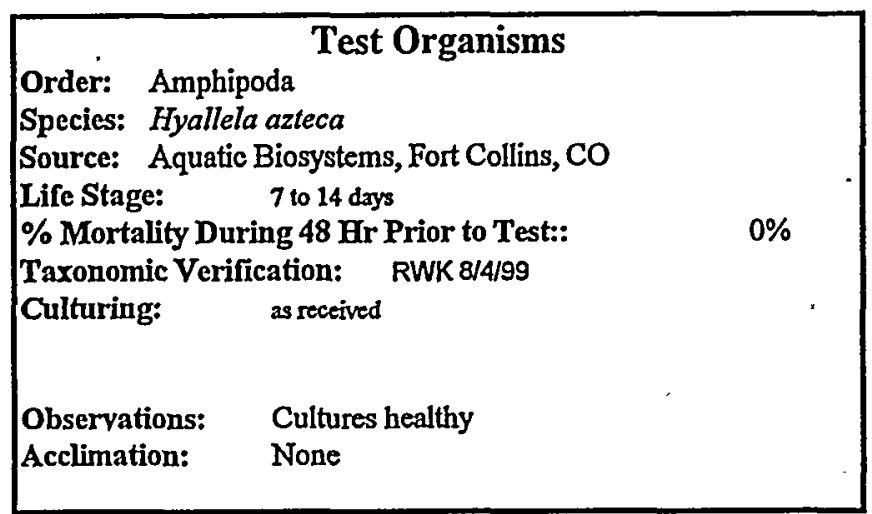

Source: BGCH5
Collection Date: $7 / 19 / 99$
Preservation: kept at $0-4^{\circ} \mathrm{C}$ in plastic
Date/Time Added to Test Chambers:
Observations:
Collection Method: grab
Disposal:

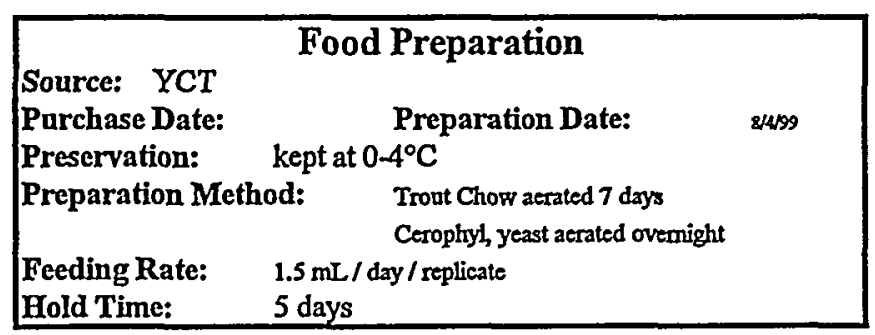

\begin{tabular}{|ccl|}
\hline \multicolumn{3}{|c|}{ Dilution Water } \\
Type: $\quad$ Surface Water & Initial & \\
Collection Date: . 8/4/99 & 10.4 & $\mathrm{mg/L}$ \\
Alkalinity & 11.4 & $\mathrm{mgl}$ \\
Hardness & 285 & unos/cm \\
Conductivity & 7.63 & units \\
pH: & & \\
Preparation Method: & & \\
Grab sample & & \\
& & \\
\hline
\end{tabular}

Source: D-7

Collection Date: 7/19/99 Homogenized?: yes

Preservation: kept at $04^{\circ} \mathrm{C}$ in plastic

Date/Time Added to Test Chambers: 8/399

Observations:

Collection Method: grab

Shipment: overnight

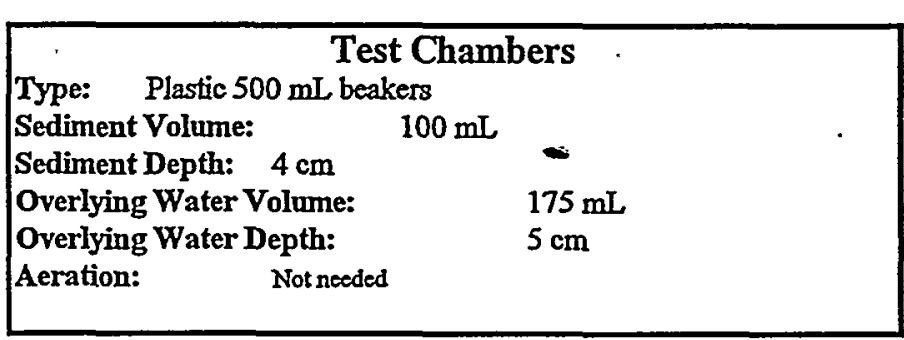




\section{CHRONIC DEFINITIVE SEDIMENT 10 DAY SURVIVAL TEST}

\section{Survival Results}

Client:

Sample Identification:

Test Date:

WSRC

August 4, 1999

\begin{tabular}{|c|c|c|c|c|c|c|}
\hline \multicolumn{7}{|l|}{ Survival in } \\
\hline Day & Rep. A & Rep B & Rep. C & Rep. D & & Mean \% \\
\hline 0 & 10 & 10 & 10 & 10 & & $100 \%$ \\
\hline & & & & & & \\
\hline & & & & & & \\
\hline & & & & & & \\
\hline & & & & & & \\
\hline & & & & & & \\
\hline & & & & & & \\
\hline 10 & 3 & 3 & 8 & 4 & & $45 \%$ \\
\hline \#Dead & 7 & 7 & 2 & 6 & & $55 \%$ \\
\hline \# Lost & 0 & 0 & 0 & 0 & & $0 \%$ \\
\hline
\end{tabular}

\begin{tabular}{|c|c|c|c|c|c|c|}
\hline Survival at & \multicolumn{7}{|c|}{ 25\% sediment D-7 F/ 75\% sediment BGCHS } & \\
\hline Day & Rep. A & Rep B & Rep. C & Rep. D & & Mean \% \\
\hline 0 & 10 & 10 & 10 & 10 & & $100 \%$ \\
\hline & & & & & & \\
\hline & & & & & & \\
\hline & & & & & & \\
\hline & & & & & & \\
\hline & & & & & & \\
\hline 10 & 4 & 6 & 5 & 10 & & $63 \%$ \\
\hline \# Dead & 6 & 4 & 5 & 0 & & $38 \%$ \\
\hline \# Lost & 0 & 0 & 0 & 0 & & $0 \%$ \\
\hline
\end{tabular}

\begin{tabular}{|c|c|c|c|c|c|c|}
\hline \multicolumn{7}{|l|}{ Survival at } \\
\hline Day & Rep. A & Rep B & Rep. C & Rep. D & & Mean \% \\
\hline 0 & 10 & 10 & 10 & 10 & & $100 \%$ \\
\hline & & & & & & \\
\hline & & & & & & \\
\hline & & & & & & \\
\hline & & & & & & \\
\hline & & & & & & \\
\hline & & & & & & \\
\hline 10 & 0 & 4 & 2 & 3 & & $23 \%$ \\
\hline \# Dead & 10 & 6 & 8 & 7 & & $78 \%$ \\
\hline \# Lost & 0 & 0 & 0 & 0 & & $0 \%$ \\
\hline
\end{tabular}

County:

NPDES\#:

ETT \#:

\begin{tabular}{|c|c|c|c|c|c|c|}
\hline Survival at & \multicolumn{7}{|c|}{$12.5 \%$ sediment D-7 w/ 87.5\% sediment BGCH5 } \\
\hline Day & Rep. A & Rep B & Rep. C & Rep. D & & Mean \% \\
\hline 0 & 10 & 10 & 10 & 10 & & $100 \%$ \\
\hline & & & & & & \\
\hline & & & & & & \\
\hline & & & & & & \\
\hline & & & & & & \\
\hline & & & & & & \\
\hline 10 & 4 & 6 & 8 & 0 & & $45 \%$ \\
\hline \#Dead & 6 & 4 & 2 & 10 & & $55 \%$ \\
\hline \#Lost & 0 & 0 & 0 & 0 & & $0 \%$ \\
\hline
\end{tabular}

\begin{tabular}{|c|c|c|c|c|c|c|}
\hline Survival at & \multicolumn{7}{|c|}{ 50\% sediment D-7 w/ 50\% sediment BGCH5 } & \\
\hline Day & Rep. A & Rep B & Rep. C & Rep. D & & Mean \% \\
\hline 0 & 10 & 10 & 10 & 10 & & $100 \%$ \\
\hline & & & & & & \\
\hline & & & & & & \\
\hline & & & & & & \\
\hline & & & & & & \\
\hline & & & & & & \\
\hline & & & & & & \\
\hline 10 & 4 & 5 & 2 & 1 & & $30 \%$ \\
\hline \# Dead & 6 & 5 & 8 & 9 & & $70 \%$ \\
\hline \# Lost & 0 & 0 & 0 & 0 & & $0 \%$ \\
\hline
\end{tabular}

\begin{tabular}{|c|c|c|c|c|c|}
\hline \multicolumn{6}{|c|}{ Survival at } \\
\hline Day & Rep. A & $\operatorname{Rep} B$ & Rep. C & Rep. D & Mean \% \\
\hline \multicolumn{6}{|l|}{0} \\
\hline \multicolumn{6}{|l|}{1} \\
\hline \multicolumn{6}{|l|}{2} \\
\hline \multicolumn{6}{|l|}{3} \\
\hline \multicolumn{6}{|l|}{4} \\
\hline \multicolumn{6}{|l|}{5} \\
\hline \multicolumn{6}{|l|}{6} \\
\hline 7 & & & & 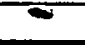 & \\
\hline \multicolumn{6}{|l|}{ \#Dead } \\
\hline \# Lost & & & & & \\
\hline
\end{tabular}




\section{CHRONIC DEFINITIVE SEDIMENT 10 DAY SURVIVAL TEST \\ Statistical Analyses - Survival}

Client: WSRC

Sample Identification: $\quad$ D-7

Test Date: $\quad$ August 4, 1999

\begin{tabular}{|ccc|}
\hline Test for Normality & & \\
& & \\
Test Used: Shapiro-Wilks & critical & 0.971 \\
& & 0.884 \\
The data are normal in distribution & \\
\end{tabular}

\section{Test for Homogeneity of Variance}

Test Used: Bartlett's Test

$\begin{array}{cr}B= & 1.52 \\ \text { critical } & 15.086\end{array}$

The data are homogeneous in variance

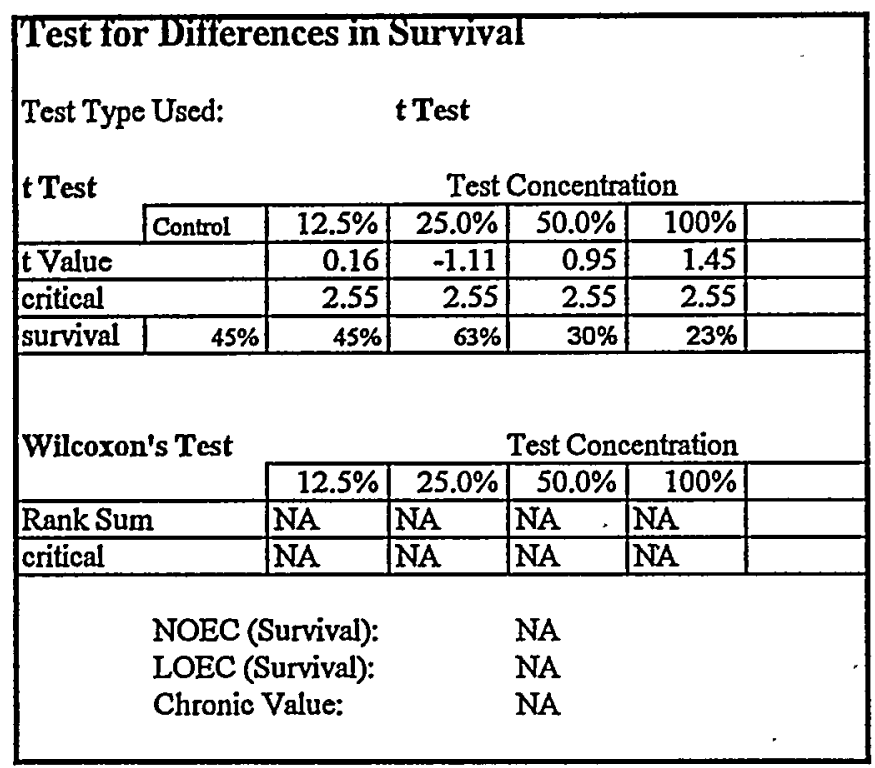

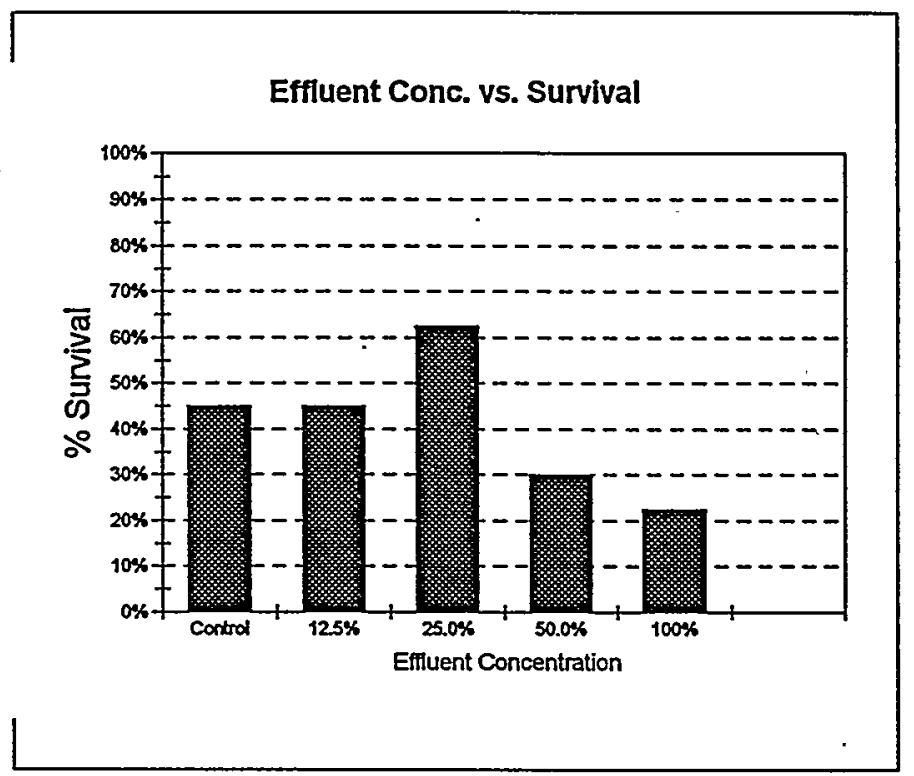

Summary Both the control sediment and the test sediment reduced survival.

Survival in $100 \%$ control sediment was not significantly less than in the control sediment. 


\section{CHRONIC DEFINITIVE SEDIMENT 10 DAY SURVIVAL TEST}

\section{Water Chemistry}

Client: WSRC

Sample Identification: $\quad$ D-7

Test Date: $\quad$ August 4, 1999

Control Sediment

\begin{tabular}{|l|r|r|r|r|r|r|}
\hline & Alkal. & Hard. & Cond. & NH3 & & \\
\hline & $\mathrm{mg} / \mathrm{L}$ & $\mathrm{mg} / \mathrm{L}$ & umhos & $\mathrm{mg} / \mathrm{L}$ & & \\
\hline Initial & 10.4 & 11.4 & 285 & $<0.1$ & & \\
\hline Day 10 & 88.2 & 49.4 & 175 & $<0.2$ & & \\
\hline
\end{tabular}

Temperature

\begin{tabular}{|c|c|c|c|c|c|c|}
\hline & \multicolumn{6}{|c|}{ Test Concentration } \\
\hline & Control & $12.50 \%$ & $25.00 \%$ & $50.00 \%$ & & $100 \%$ \\
\hline Initial & $\overline{22.3}$ & & $\overline{22.3}$ & & & 22.3 \\
\hline Day 1 & 20.3 & & 20.5 & & & 20.5 \\
\hline Day 2 & $\overline{22.6}$ & & 22.0 & & & 22.0 \\
\hline Day 3 & $\overline{22.2}$ & & 21.8 & & $\%$ & $\overline{22.0}$ \\
\hline Day 4 & 20.2 & & 20.2 & & 8 & 20.2 \\
\hline Day 5 & 21.6 & & 21.5 & & & 21.5 \\
\hline Day 6 & 22.2 & & 22.2 & & & 22.2 \\
\hline Day 7 & 21.2 & & $\overline{21.2}$ & & $\%$ & 21.2 \\
\hline Day 8 & 20.8 & & 20.8 & $1 \%$ & א. & 20.8 \\
\hline Day 9 & 22.0 & & 21.6 & & & 21.6 \\
\hline Day 10 & 21.0 & & 21.0 & & & 21.0 \\
\hline
\end{tabular}

Test Sediment

\begin{tabular}{|r|r|r|r|r|r|r|}
\hline & Alkal. & Hard. & Cond. & $\mathrm{NH3}$ & & \\
\hline & $\mathrm{mg} / \mathrm{L}$ & $\mathrm{mg} / \mathrm{L}$ & umhos & $\mathrm{mg} / \mathrm{L}$ & & \\
\hline Initial & 10.4 & 11.4 & 285 & $<0.1$ & & \\
\hline Day 10 & 10.9 & 5.7 & 78 & $<0.1$ & & \\
\hline
\end{tabular}

Dissolved Oxygen

\begin{tabular}{|c|c|c|c|c|c|}
\hline & \multicolumn{5}{|c|}{ Test Concentration } \\
\hline & Control & $12.50 \%$ & $25.00 \%$ & $50.00 \%$ & $100 \%$ \\
\hline Initial & 8.0 & & 8.0 & & 8.0 \\
\hline Day 1 & 8.1 & & 7.6 & & 7.9 \\
\hline Day 2 & 7.8 & & 8.0 & & 8.0 \\
\hline Day 3 & 7.6 & & 7.8 & & 7.9 \\
\hline Day 4 & $\overline{7.4}$ & & 7.6 & & $\overline{7.4}$ \\
\hline Day 5 & 7.1 & & 7.4 & & $\overline{7.4}$ \\
\hline Day 6 & 7.4 & & 7.3 & & 7.4 \\
\hline Day 7 & 7.2 & & 7.0 & & 7.0 \\
\hline Day 8 & 7.1 & & 7.4 & & $\overline{7.2}$ \\
\hline Day 9 & 7.8 & & 7.5 & & 7.7 \\
\hline Day 10 & 7.7 & & & & 7.7 \\
\hline
\end{tabular}

Test Results Reviewed and Approved By:

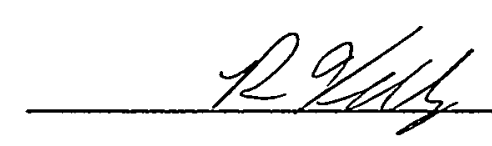




\title{
10 DAY AMPHIPOD SEDIMENT TOXICITY TEST
}

\author{
EPA/600/R-94/024 Method 100.1
}

Test Organism: Hyallela azteca

\author{
Client: WSRC \\ Sample Identification: $\quad$ D-28
}

Test Start Date: $\quad$ 8/4/99 


\section{DAY AMPHIPOD SEDIMENT TOXICITY TEST}

\begin{tabular}{|l|l|l|}
\hline Lab W\# & Client: $\quad$ WSRC & Start Date: $8 / 4 / 99$ \\
\cline { 2 - 4 } T12312 & Sample Identification: D-28 & End Date: $8 / 14 / 99$ \\
\hline
\end{tabular}

\section{Test System}

EPA/600/R-94/024 Method 100.1

The test was set as a Pass/Fail test with a control treatment and one sample treatment. Each treatment replicate consisted of $100 \mathrm{~g}$ of sediment and $175 \mathrm{~mL}$ of dilution water. There were eight replicates for each treatment. Ten test organisms were placed in each replicate. Sediment from a local stream was used in the control treatment. Test organisms were fed with YCT food. The test was conducted at a temperature of $23^{\circ} \mathrm{C}$ and a light cycle of $16 \mathrm{hr}$ light/8 hr dark. Test vessels were $500 \mathrm{~mL}$ plastic beakers placed in a constant temperature incubator room.

\begin{tabular}{|c|c|c|}
\hline \multicolumn{3}{|c|}{$\begin{aligned} \text { Test Organisms } \\
\end{aligned}$} \\
\hline \multicolumn{3}{|c|}{$\begin{array}{l}\text { Order: Amphipoda } \\
\text { Species: Hyallela azteca } \\
\text { Source: }\end{array}$} \\
\hline $\begin{array}{l}\text { Life Stage: } \\
\% \text { Mortality D } \\
\text { Taxonomic Ve }\end{array}$ & $\begin{array}{l}7 \text { to } 14 \text { days } \\
\text { ring } 48 \text { Hr Prior to Test:: } \\
\text { fication: RWK } 8 / 4 / 99\end{array}$ & $0 \%$ \\
\hline Culturing: & as received & \\
\hline $\begin{array}{l}\text { Observations: } \\
\text { Acclimation: }\end{array}$ & $\begin{array}{l}\text { Cultures healthy } \\
\text { None }\end{array}$ & \\
\hline
\end{tabular}

Control Sediment
Source: Resurrection Creek
Collection Date: $8 / 4 / 99$
Preservation: kept at $0-4^{\circ} \mathrm{C}$ in plastic
Date/Time Added to Test Chambers: 83/99
Observations: silt and organic
Collection Method: grab
Disposal

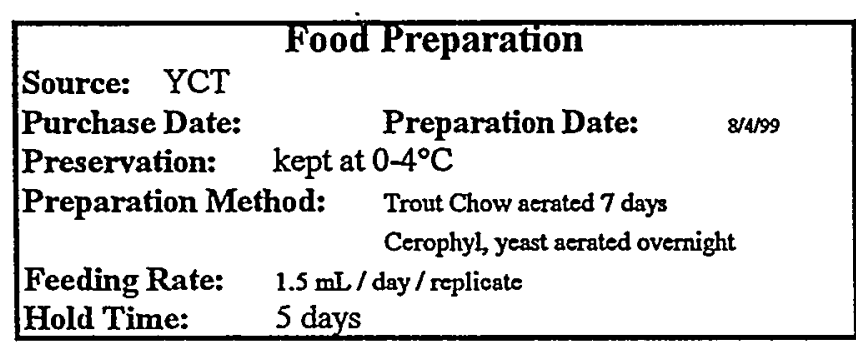

\begin{tabular}{|ccc|}
\hline \multicolumn{3}{c|}{ Dilution Water } \\
Type: $\quad$ Surface Water & & \\
Collection Date $8 / 4 / 99$ & Initial & \\
Alkalinity & 10.4 & $\mathrm{mg} /$ \\
Hardness & 11.4 & $\mathrm{mg/L}$ \\
Conductivity & 285 & umboedcm \\
$p H:$ & 7.63 & units \\
& & \\
Preparation Method: & & \\
Grab sample & & \\
& & \\
\end{tabular}

Source: D-28

Collection Date 7/16/99 Homogenized?: yes

Preservation: kept at $0-4^{\circ} \mathrm{C}$ in plastic

Date/Time Added to Test Chambers: $8 / 3 / 99$

Observations:

Collection Method: grab

Shipment: overnight

\begin{tabular}{l} 
Test Chambers \\
Type: Plastic $500 \mathrm{~mL}$ beakers \\
Sediment Volume: $\quad 100 \mathrm{~mL}$ - \\
Sediment Depth $4 \mathrm{~cm}$ \\
Overlying Water Volume: $\quad 175 \mathrm{~mL}$ \\
Overlying Water Depth: $\quad 5 \mathrm{~cm}$ \\
Aeration: Not needed \\
\hline
\end{tabular}




\section{DAY AMPHIPOD SEDIMENT TOXICITY TEST}

\begin{tabular}{|l|l|l|}
\hline Lab W\# & Client: $\quad$ WSRC & Start Date: $8 / 4 / 99$ \\
\cline { 2 - 3 } T12312 & Sample Identification: D-28 & End Date: $8 / 14 / 99$ \\
\hline
\end{tabular}

\section{WATER CHEMISTRY TEST METHODS}

\begin{tabular}{|l|l}
\hline Temperature: SM 18th Ed. 2550 & Alkalinity: SM 18th Ed. 2320 \\
Dissolved Oxygen: SM 18th Ed. 4500-O & Hardness: SM 18th Ed. 2340 \\
pH: SM 18th Ed. 4500-H+ & Conductivity: SM 18th Ed. 2510 \\
\hline
\end{tabular}

\section{WATER CHEMISTRY TEST DATA - OVERLYING WATER}

CONTROL TREATMENT

\begin{tabular}{|c|c|c|c|c|c|c|}
\hline & Temp. & $\mathrm{pH}$ & D.O. & D.O. & Alkalinity & Ammonia \\
\hline Test Day & C & units & $\mathrm{mg} / \mathrm{L}$ & $\%$ sat. & Initial & Initial \\
\hline Start & 22.3 & 7.63 & 8.0 & $92 \%$ & 10.4 & $<0.20$ \\
\hline 1 & 20.5 & & 7.9 & $87 \%$ & Final & Final \\
\hline 2 & 22.6 & & 7.6 & $87 \%$ & 88.2 & $<0.1$ \\
\hline 3 & 22.0 & & 7.5 & $86 \%$ & $m g h$ & $\mathrm{mg} \Omega$ \\
\hline 4 & 20.2 & & 7.8 & $86 \%$ & Cond. & Hardness \\
\hline 5 & 21.5 & & 7.0 & $79 \%$ & Initial & Initial \\
\hline 6 & 22.2 & & 7.2 & $83 \%$ & 285 & 11.4 \\
\hline 7 & 21.2 & & 6.8 & $76 \%$ & Frnal & Final. \\
\hline 8 & 20.8 & & 6.8 & $75 \%$ & 175.0 & 49.4 \\
\hline 9 & 20.2 & & 8.0 & $88 \%$ & umhos/cm & $\mathrm{mg} \Omega$ \\
\hline 10 & 21.0 & 7.46 & 4.9 & $55 \%$ & Aeration: $\mathrm{n}$ & \\
\hline
\end{tabular}

SAMPLE TREATMENT

\begin{tabular}{|c|c|c|c|c|c|c|}
\hline & Temp. & $\mathrm{pH}$ & D.O. & D.O. & Alkalinity & Ammonia \\
\hline Test Day & C & units & $\mathrm{mg} / \mathrm{L}$ & \% sat. & Initial & Initial \\
\hline Start & 22.3 & 7.63 & 8.0 & $92 \%$ & 10.4 & $<0.20$ \\
\hline 1 & 20.5 & & 7.7 & $85 \%$ & Final & Finel \\
\hline 2 & 22.6 & & 7.8 & $90 \%$ & 52.6 & $<0.1$ \\
\hline 3 & 22.0 & & 7.3 & $84 \%$ & $\mathrm{mgh}$ & $\mathrm{mgl}$ \\
\hline 4 & 20.2 & & 7.4 & $81 \%$ & Cond. & Hardness \\
\hline 5 & 21.5 & & 7.1 & $80 \%$ & Initial & Initisl \\
\hline 6 & 22.2 & & 7.3 & $84 \%$ & 285 & 11.4 \\
\hline 7 & 21.2 & & 7.6 & $85 \%$ & Final & Final \\
\hline 8 & 20.8 & & 7.2 & $79 \%$ & 42.0 & 15.2 \\
\hline 9 & 20.2 & & 8.4 & $92 \%$ & & $m g h$ \\
\hline 10 & 21.0 & 7.18 & 8.4 & $94 \%$ & Aeration: $\Omega$ & \\
\hline
\end{tabular}

\section{SURVIVAL AND GROWTH RESULTS}

CONTROL TREATMENT

\begin{tabular}{|c|c|c|c|c|c|c|}
\hline & \multicolumn{3}{|c|}{ \# Organisms } & \multirow[b]{2}{*}{$\begin{array}{c}\% \\
\text { Survival }\end{array}$} & \multirow{2}{*}{$\begin{array}{l}\text { Mean } \\
\text { Length } \\
\text { (mm) }\end{array}$} & \multirow{2}{*}{$\begin{array}{l}\text { Standard } \\
\text { Deviation }\end{array}$} \\
\hline & Initial & $\begin{array}{c}\text { Live at } \\
\text { End }\end{array}$ & $\begin{array}{l}\text { Dead } \\
\text { at End }\end{array}$ & & & \\
\hline $\bar{A}$ & 10 & 10 & 0 & $100 \%$ & & \\
\hline $\mathrm{B}$ & 10 & 6 & 4 & $60 \%$ & & \\
\hline $\bar{C}$ & 10 & 9 & 1 & $90 \%$ & & \\
\hline $\bar{D}$ & 10 & 8 & 2 & $80 \%$ & & \\
\hline$\overline{\mathrm{E}}$ & 10 & 10 & 0 & $100 \%$ & & \\
\hline $\mathrm{F}$ & 10 & 10 & 0 & $100 \%$ & & \\
\hline $\mathbf{G}$ & 10 & 5 & 5 & $50 \%$ & & \\
\hline $\mathrm{H}$ & 10 & 9 & 1 & $90 \%$ & & \\
\hline Mean & 10.0 & & & $84 \%$ & & \\
\hline
\end{tabular}

SAMPLE TREATMENT

\begin{tabular}{|c|c|c|c|c|c|c|}
\hline & \multicolumn{3}{|c|}{ \# Organisms } & \multirow[b]{2}{*}{$\begin{array}{c}\% \\
\text { Survival }\end{array}$} & \multirow{2}{*}{$\begin{array}{l}\text { Mean } \\
\text { Length } \\
\text { (mm) }\end{array}$} & \multirow{2}{*}{$\begin{array}{l}\text { Standard } \\
\text { Deviation }\end{array}$} \\
\hline & Initial & $\begin{array}{c}\text { Live at } \\
\text { End }\end{array}$ & $\begin{array}{l}\text { Dead } \\
\text { at End }\end{array}$ & & & \\
\hline $\bar{A}$ & 10 & 4 & 6 & $40 \%$ & & \\
\hline B & 10 & 8 & 2 & $80 \%$ & - & \\
\hline C & 10 & 10 & 0 & $100 \%$ & & \\
\hline D & 10 & 5 & 5 & $50 \%$ & & \\
\hline $\bar{E}$ & 10 & 5 & 5 & $50 \%$ & & \\
\hline $\bar{F}$ & 10 & 5 & 5 & $50 \%$ & & \\
\hline $\mathrm{G}$ & 10 & 8 & 2 & $80 \%$ & & \\
\hline $\mathrm{H}$ & 10 & 3 & 7 & $30 \%$ & & \\
\hline Mean & 10.0 & & & $60 \%$ & & \\
\hline
\end{tabular}




\section{DAY AMPHIPOD SEDIMENT TOXICITY TEST}

\begin{tabular}{|c|c|c|c|c|}
\hline \multirow{2}{*}{$\begin{array}{l}\text { Lab I\# } \\
\text { T12312 }\end{array}$} & Client: & & Start Date: & $8 / 4 / 99$ \\
\hline & Sample Identification: & $D-28$ & End Date: & $8 / 14 / 99$ \\
\hline
\end{tabular}

\section{RESULTS}

\begin{tabular}{|l|r|}
\hline \multicolumn{2}{|c|}{$\begin{array}{c}\text { SURVTVAL DATA } \\
\text { Mean of Eight Replicates }\end{array}$} \\
\cline { 2 - 2 } & Mean \% \\
\hline Control & $83.8 \%$ \\
\hline Sample & $60.0 \%$ \\
\hline
\end{tabular}

\begin{tabular}{|l|l|}
\hline \multicolumn{2}{|c|}{$\begin{array}{l}\text { GROWTH DATA } \\
\text { Mean Length (mm) }\end{array}$} \\
\cline { 2 - 2 } & Mean \\
\hline Control & NA \\
\hline Sample & NA \\
\hline
\end{tabular}

\section{STATISTICAL ANALYSES}

\begin{tabular}{|c|c|c|}
\hline \multicolumn{3}{|c|}{ SURVIVAL DATA } \\
\hline Test for Normality: & $W=$ & 0.96 \\
\hline \multicolumn{2}{|c|}{ Critical value: } & 0.84 \\
\hline \multicolumn{3}{|c|}{ Data are normal in distribution } \\
\hline \multicolumn{3}{|c|}{ Test for Homogeneity of Variance: } \\
\hline \multicolumn{3}{|c|}{1.13} \\
\hline \multicolumn{3}{|c|}{ Critical value: } \\
\hline \multicolumn{3}{|c|}{ Data are homogeneous in variance } \\
\hline \multicolumn{2}{|c|}{ Test for Differences in Survival: } & "t" Test \\
\hline & $t=2.09$ & \\
\hline \multicolumn{3}{|c|}{ Critical value: $\quad 1.75$} \\
\hline \multicolumn{3}{|c|}{$\begin{array}{l}\text { The sediment significantly reduced survival } \\
\text { of the test organisms. }\end{array}$} \\
\hline
\end{tabular}

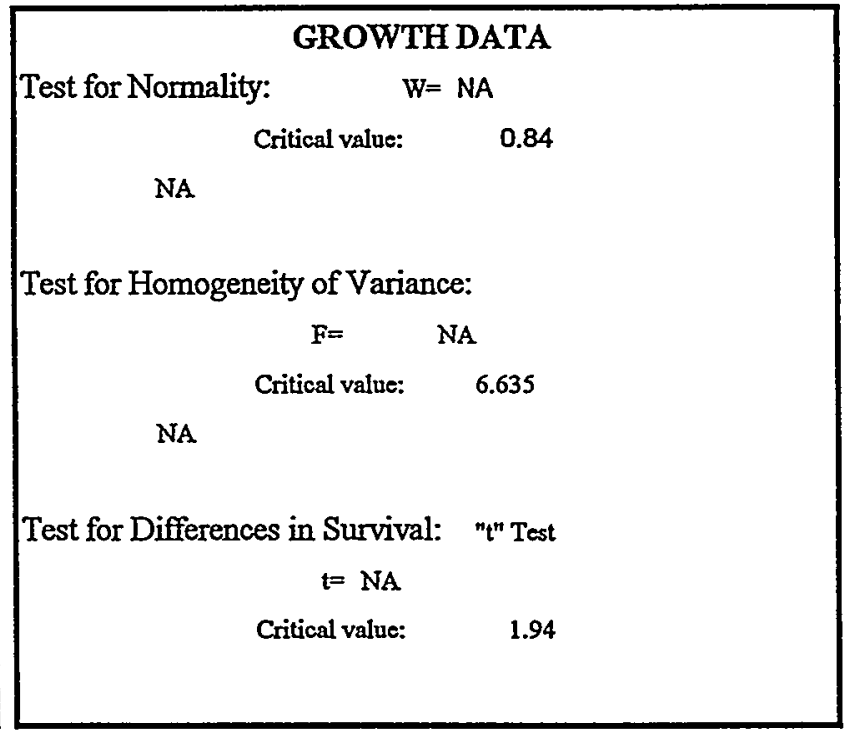

\section{COMMENTS}

None

\begin{tabular}{ll}
\hline Principal Analyst: & Marsha Leitzel \\
Report Reviewed By: & Robert W. Kelley, Ph.D. \\
\hline
\end{tabular}

Signature:

Signature:

Date: $10-19-99$

Date: $10-19-99$

\section{CONCLUSIONS}

The sediment reduces the survival of the test organisms. 


\title{
10 DAY AMPHIPOD SEDIMENT TOXICITY TEST
}

\author{
EPA/600/R-94/024 Method 100.1
}

Test Organism: Hyallela azteca

\author{
Client: WSRC \\ Sample Identification: TNSED25
}

Test Start Date: $\quad 8 / 4 / 99$ 


\section{DAY AMPHIPOD SEDIMENT TOXICITY TEST}

\begin{tabular}{|l|l|l|}
\hline Lab D\#\# & Client: $\quad$ WSRC & Start Date: $8 / 4 / 99$ \\
\cline { 2 - 4 } T12310 & Sample Identification: TNSED25 & End Date: $8 / 14 / 99$ \\
\hline
\end{tabular}

Test System

EPA/600/R-94/024 Method 100.1

The test was set as a Pass/Fail test with a control treatment and one sample treatment. Each treatment replicate consisted of $100 \mathrm{~g}$ of sediment and $175 \mathrm{~mL}$ of dilution water. There were eight replicates for each treatment. Ten test organisms were placed in each replicate. Sediment from a local stream was used in the control treatment. Test organisms were fed with YCT food. The test was conducted at a temperature of $23^{\circ} \mathrm{C}$ and a light cycle of $16 \mathrm{hr}$ light/8 hr dark. Test vessels were $500 \mathrm{~mL}$ plastic beakers placed in a constant temperature incubator room.

\begin{tabular}{|l|}
\hline \multicolumn{3}{|c|}{ Test Organisms } \\
Order: Amphipoda \\
Species: Hyallela azteca \\
Source: Aquatic Biosystems, Fort Collins, CO \\
Life Stage: $\quad 7$ to 14 days \\
\% Mortality During 48 Hr Prior to Test:: $\quad 0 \%$ \\
Taxonomic Verification: RWK 8/4/99 \\
Culturing: as received \\
\\
Observations: Cultures healthy \\
Acclimation: $\quad$ None
\end{tabular}

\section{Test Organisms}

Order: Amphipoda

Source: Aquatic Biosystems, Fort Collins, $\mathrm{CO}$

Life Stage: $\quad 7$ to 14 days

\% Mortality During $48 \mathrm{Hr}$ Prior to Test::

Taxonomic Verification: RWK 8/4/99

Culturing: as received

Observations: Cultures healthy

\begin{tabular}{|l|}
\hline \multicolumn{1}{|c|}{ Control Sediment } \\
Source: Resurrection Creek \\
Collection Date: $8 / 4 / 99$ \\
Preservation: kept at $0-4^{\circ} \mathrm{C}$ in plastic \\
Date/Time Added to Test Chambers: 83/99 \\
Observations: silt and organic \\
Collection Method: grab \\
Disposal \\
\hline
\end{tabular}

\begin{tabular}{|c|c|c|c|}
\hline \multicolumn{4}{|c|}{ Food Preparation } \\
\hline $\begin{array}{l}\text { Source: YCT } \\
\text { Purchase Date: } \\
\text { Preservation: }\end{array}$ & kept & $\begin{array}{l}\text { Preparation Date: } \\
t 0-4^{\circ} \mathrm{C}\end{array}$ & $8 / 499$ \\
\hline Preparation $\mathrm{Me}$ & thod: & \multicolumn{2}{|c|}{$\begin{array}{l}\text { Trout Chow aeraled } 7 \text { days } \\
\text { Cerophyl, yeast aerated overnight }\end{array}$} \\
\hline $\begin{array}{l}\text { Feeding Rate: } \\
\text { Hold Time: }\end{array}$ & \multicolumn{3}{|c|}{$\begin{array}{l}1.5 \mathrm{~mL} / \text { day } / \text { replicate } \\
5 \text { days }\end{array}$} \\
\hline
\end{tabular}

\begin{tabular}{|c|c|c|}
\hline \multicolumn{3}{|c|}{ Dilution Water } \\
\hline \multicolumn{3}{|l|}{ Type: $\quad$ Surface Water } \\
\hline \multirow{5}{*}{$\begin{array}{l}\text { Collection Date } 8 / 4 / 99 \\
\text { Alkalinity } \\
\text { Hardness } \\
\text { Conductivity } \\
\text { pH: }\end{array}$} & Initial & \\
\hline & 10.4 & $\mathrm{mg} / \mathrm{L}$ \\
\hline & 11.4 & $\mathrm{mg} / \mathrm{L}$ \\
\hline & 285 & unhoy cm \\
\hline & 7.63 & units \\
\hline \multicolumn{3}{|l|}{$\begin{array}{l}\text { Preparation Method: } \\
\text { Grab sample }\end{array}$} \\
\hline \multicolumn{3}{|c|}{ Test Sediment } \\
\hline \multirow{7}{*}{\multicolumn{2}{|c|}{$\begin{array}{l}\text { Source: TNSED } 25 \\
\text { Collection Date } 7 / 16 / 99 \quad \text { Homogenized?: } \\
\text { Preservation: kept at } 0-4^{\circ} \mathrm{C} \text { in plastic } \\
\text { Date/Time Added to Test Chambers: } 83 / 99 \\
\text { Observations: } \\
\text { Collection Method: grab } \\
\text { Shipment: overnight }\end{array}$}} & \\
\hline & & yes \\
\hline & & \\
\hline & & \\
\hline & & \\
\hline & & \\
\hline & & \\
\hline
\end{tabular}

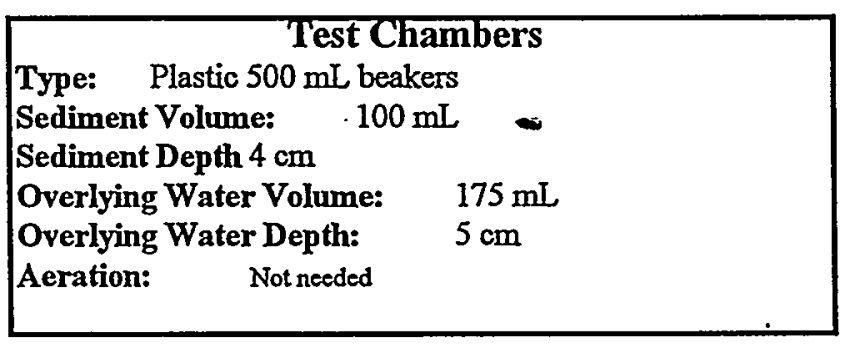




\section{DAY AMPHIPOD SEDIMENT TOXICITY TEST}

\begin{tabular}{|l|l|l|}
\hline Lab WH & Client: $\quad$ WSRC & Start Date: $8 / 4 / 99$ \\
\cline { 2 - 3 } T12310 & Sample Identification: TNSED25 & End Date: $8 / 14 / 99$ \\
\hline
\end{tabular}

WATER CHEMISTRY TEST METHODS

Temperature: SM 18th Ed. 2550

Dissolved Oxygen: SM 18th Ed. 4500-O

pH: SM 18th Ed. 4500-H+
Alkalinity: SM 18th Ed. 2320

Hardness: SM 18th Ed. 2340

Conductivity: SM 18th Ed. 2510

\section{WATER CHEMISTRY TEST DATA - OVERLYING WATER}

CONTROL TREATMENT

\begin{tabular}{|c|c|c|c|c|c|c|}
\hline & Temp. & $\mathrm{pH}$ & D.O. & D.O. & Alkslinity & Ammonia \\
\hline Test Day & C & units & $\mathrm{mg} / \mathrm{L}$ & $\%$ sat. & Initial & Initial \\
\hline Start & 22.3 & 7.63 & 8.0 & $92 \%$ & 10.4 & $<0.20$ \\
\hline 1 & 20.5 & & 7.9 & $87 \%$ & Firal & Final \\
\hline 2 & 22.6 & & 7.6 & $87 \%$ & 88.2 & $<0.1$ \\
\hline 3 & 22.0 & & 7.5 & $86 \%$ & $\mathrm{mg} \Omega$ & $\mathrm{mgh}$ \\
\hline 4 & 20.2 & & 7.8 & $86 \%$ & Cond. & Hardness \\
\hline 5 & 21.5 & & 7.0 & $79 \%$ & Initial & Initial \\
\hline 6 & 22.2 & & 7.2 & $83 \%$ & 285 & 11.4 \\
\hline 7 & 21.2 & & 6.8 & $76 \%$ & Final & Frnal \\
\hline 8 & 20.8 & & 6.8 & $75 \%$ & 175.0 & 49.4 \\
\hline 9 & 20.2 & & 8.0 & $88 \%$ & umhos $/ \mathrm{cm}$ & $\mathrm{mg} \Omega$ \\
\hline 10 & 21.0 & 7.46 & 4.9 & $55 \%$ & \multicolumn{2}{|c|}{ Aeration: none } \\
\hline
\end{tabular}

SAMPLE TREATMENT

\begin{tabular}{|c|c|c|c|c|c|c|}
\hline & Temp. & $\mathrm{pH}$ & D.o. & D.o. & Alkalinity & Ammonia \\
\hline Test Day & C & units & $\mathrm{mg} / \mathrm{L}$ & \% sat. & Initial & Initial \\
\hline Start & 22.3 & 7.63 & 8.0 & $92 \%$ & 10.4 & $<0.20$ \\
\hline 1 & 20.2 & & 7.9 & $87 \%$ & Final & Final \\
\hline 2 & 22.6 & & $\overline{8.0}$ & $92 \%$ & 9.6 & 0.27 \\
\hline 3 & 22.0 & & $\overline{7.7}$ & $89 \%$ & $\mathrm{mg} \Omega$ & $\mathrm{mgh}$ \\
\hline 4 & 20.2 & & $\overline{7.4}$ & $81 \%$ & Cond. & Hardness \\
\hline 5 & 21.5 & & 7.3 & $82 \%$ & Initial & Initial \\
\hline 6 & 22.2 & & $\overline{7.4}$ & $85 \%$ & 285 & 11.4 \\
\hline 7 & $\overline{21.2}$ & & 7.7 & $87 \%$ & Frall & Final \\
\hline 8 & 20.8 & & 7.6 & $84 \%$ & 45.0 & 1.9 \\
\hline 9 & 20.1 & & $\overline{7.7}$ & $85 \%$ & umhos $/ \mathrm{cm}$ & mgn \\
\hline 10 & 21.0 & 7.17 & $\overline{8.1}$ & $91 \%$ & Aeration: n & \\
\hline
\end{tabular}

\section{SURVIVAL AND GROWTH RESULTS}

CONTROL TREATMENT

\begin{tabular}{|c|c|c|c|c|c|c|}
\hline & \multicolumn{3}{|c|}{ \# Organisms } & \multirow[b]{2}{*}{$\begin{array}{c}\% \\
\text { Survival }\end{array}$} & \multirow{2}{*}{$\begin{array}{l}\text { Mean } \\
\text { Length } \\
\text { (mm) }\end{array}$} & \multirow{2}{*}{$\begin{array}{l}\text { Standard } \\
\text { Deviation }\end{array}$} \\
\hline & Initial & $\begin{array}{c}\text { Live at } \\
\text { End }\end{array}$ & $\begin{array}{l}\text { Dead } \\
\text { at End }\end{array}$ & & & \\
\hline $\bar{A}$ & 10 & 10 & 0 & $100 \%$ & & \\
\hline $\bar{B}$ & 10 & 6 & 4 & $60 \%$ & & \\
\hline C & 10 & 9 & 1 & $90 \%$ & & \\
\hline $\bar{D}$ & 10 & 8 & 2 & $80 \%$ & & \\
\hline $\mathrm{E}$ & 10 & 10 & 0 & $100 \%$ & & \\
\hline $\mathrm{F}$ & 10 & 10 & 0 & $100 \%$ & & \\
\hline$G$ & 10 & 5 & 5 & $50 \%$ & & \\
\hline $\mathrm{H}$ & 10 & 9 & 1 & $90 \%$ & & \\
\hline Mean & 10.0 & & & $84 \%$ & & \\
\hline
\end{tabular}

SAMPLE TREATMENT

\begin{tabular}{|c|c|c|c|c|c|c|}
\hline & \multicolumn{3}{|c|}{ \# Organisms } & \multirow[b]{2}{*}{$\begin{array}{c}\% \\
\text { Survival }\end{array}$} & \multirow{2}{*}{$\begin{array}{l}\text { Mean } \\
\text { Length } \\
(\mathrm{mm})\end{array}$} & \multirow{2}{*}{$\begin{array}{l}\text { Standard } \\
\text { Deviation }\end{array}$} \\
\hline & Irnitial & Live at & $\begin{array}{l}\text { Dead } \\
\text { at End }\end{array}$ & & & \\
\hline $\bar{A}$ & 10 & 7 & $\overline{3}$ & $70 \%$ & & \\
\hline $\bar{B}$ & 10 & 10 & 0 & $100 \%$ & & \\
\hline $\bar{C}$ & 10 & 7 & 3 & $70 \%$ & & \\
\hline $\bar{D}$ & 10 & 3 & $\overline{7}$ & $30 \%$ & & \\
\hline $\bar{E}$ & 10 & $\overline{9}$ & $\overline{1}$ & $90 \%$ & & \\
\hline$F$ & 10 & 10 & $\overline{0}$ & $100 \%$ & & \\
\hline$\overline{\mathrm{G}}$ & 10 & $\overline{5}$ & $\overline{5}$ & $50 \%$ & & \\
\hline$\overline{\mathrm{H}}$ & 10 & 8 & 2 & $80 \%$ & & \\
\hline$\overline{\text { Mean }}$ & 10.0 & & & $74 \%$ & & \\
\hline
\end{tabular}




\section{DAY AMPHIPOD SEDIMENT TOXICITY TEST}

\begin{tabular}{|l|l|l|}
\hline Lab WH & Client: $\quad$ WSRC & Start Date: $8 / 4 / 99$ \\
\cline { 2 - 4 } T12310 & Sample Identification: TNSED25 & End Date: 8/14/99 \\
\hline
\end{tabular}

\section{RESULTS}

\begin{tabular}{|l|c|}
\hline \multicolumn{2}{|c|}{$\begin{array}{c}\text { SURVIVAL DATA } \\
\text { Mean of Eight Replicates }\end{array}$} \\
\cline { 2 - 2 } & Mean \% \\
\hline Control & $83.8 \%$ \\
\hline Sample & $73.8 \%$ \\
\hline
\end{tabular}

\begin{tabular}{|l|l|}
\hline \multicolumn{2}{|c|}{$\begin{array}{c}\text { GROWTH DATA } \\
\text { Mean Length (mm) }\end{array}$} \\
\cline { 2 - 2 } & \\
\cline { 2 - 2 } & Mean \\
\hline Control & NA \\
\hline Sample & NA \\
\hline
\end{tabular}

\section{STATISTICAL ANALYSES}

\begin{tabular}{|c|c|c|}
\hline \multicolumn{3}{|c|}{ SURVIVAL DATA } \\
\hline \multirow{2}{*}{ Test for Normality: } & $\mathrm{W}=$ & 0.95 \\
\hline & & 0.84 \\
\hline \multicolumn{3}{|c|}{ Data are normal in distribution } \\
\hline \multicolumn{3}{|c|}{ Test for Homogeneity of Variance: } \\
\hline & \multicolumn{2}{|c|}{1.32} \\
\hline & \multicolumn{2}{|r|}{6.64} \\
\hline \multicolumn{3}{|c|}{ Data are homogeneous in variance } \\
\hline \multicolumn{3}{|c|}{ Test for Differences in Survival: } \\
\hline \multicolumn{3}{|c|}{$t=0.88$} \\
\hline \multicolumn{3}{|c|}{ Critical value: $\quad 1.75$} \\
\hline \multicolumn{3}{|c|}{$\begin{array}{l}\text { The sediment did not significantly reduce survival } \\
\text { of the test organisms. }\end{array}$} \\
\hline
\end{tabular}

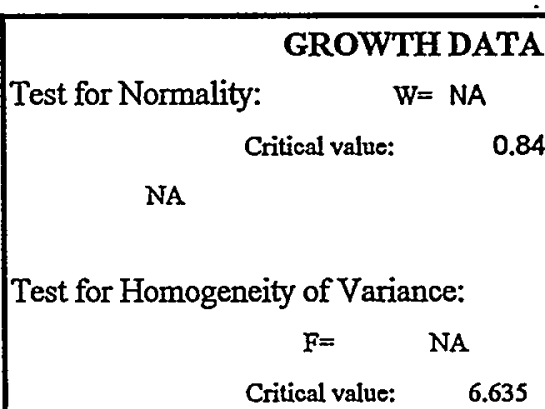

NA

Test for Differences in Survival: ${ }^{n} \mathrm{t}^{n}$ Test

$$
t=N A
$$

Critical value: $\quad 1.94$

\section{COMMENTS}

None

\begin{tabular}{|c|c|c|}
\hline $\begin{array}{l}\text { Principal Analyst: } \\
\text { Report Reviewed By: }\end{array}$ & $\begin{array}{l}\text { Marsha Leitzel } \\
\text { Robert W. Kelley, Ph.D. }\end{array}$ & 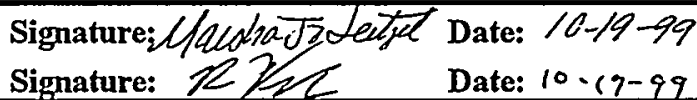 \\
\hline
\end{tabular}

CONCLUSIONS

The sediment does not reduce survival of the test organisms. 


\title{
10 DAY CHIRONOMUS SEDIMENT TOXICITY TEST
}

\author{
EPA/600/R-94/024
}

\author{
Test Organism: Chironomus tentans
}

\begin{abstract}
Client: WSRC
Sample Identification: $\quad$ BGCH5
\end{abstract}

Test Start Date: $\quad 8 / 24 / 99$ 


\section{DAY CHIRONOMUS SEDIMENT TOXICITY TEST}

\begin{tabular}{|l|l|ll|}
\hline Lab ID\# & Client: $\quad$ WSRC & Start Date: & 8/24/99 \\
\cline { 2 - 4 } T12308 & Sample Identification: $\quad$ BGCH5 & End Date: & $9 / 3 / 99$ \\
\hline
\end{tabular}

\section{Test System \\ EPA/600/R-94/024}

The test was set as a Pass/Fail test with a control treatment and one sample treatment. Each treatment replicate consisted of $100 \mathrm{~g}$ of sediment and $175 \mathrm{~mL}$ of dilution water. There were eight replicates for each treatment. Ten test organisms were placed in each replicate. Sediment from a local stream was used in the control treatment. Test organisms were fed with YCT food. The test was conducted at a temperature of $23^{\circ} \mathrm{C}$ and a light cycle of $16 \mathrm{hr}$ light/8 hr dark. Test vessels were $500 \mathrm{~mL}$ plastic beakers placed in a constant temperature incubator room.

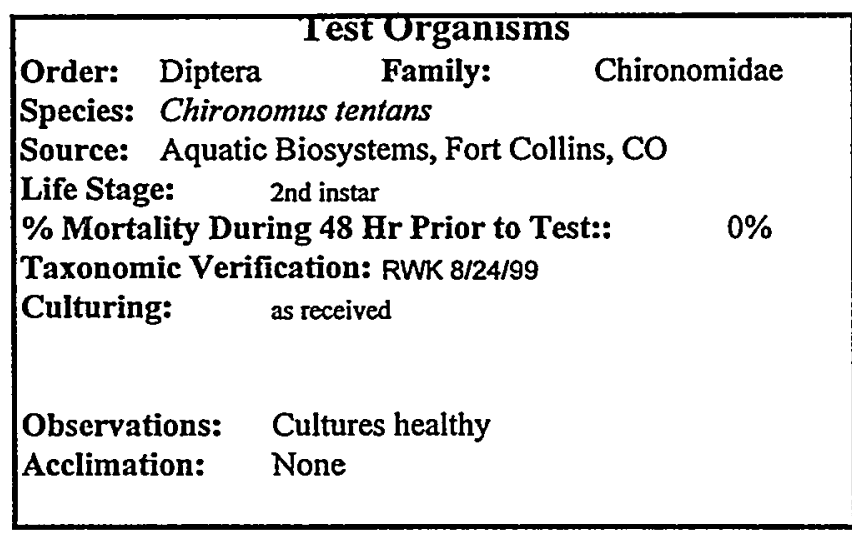

\begin{tabular}{|lcl|}
\hline \multicolumn{3}{|c|}{ DIlution Water . } \\
Type: Surface Water & & \\
Collection Date 8/2399 & Initial - & \\
Alkalinity & 10.4 & $\mathrm{mg/L}$ \\
Hardness & 11.4 & $\mathrm{mg/L}$ \\
Conductivity & 285 & umbos/cm \\
pH: & 7.63 & units \\
& & \\
Preparation Method: & & \\
Grab sample & & \\
& & \\
\end{tabular}

Control Sedment
Source: Resurrection Creek
Collection Date: $8 / 4 / 99$
Preservation: kept at $0-4^{\circ} \mathrm{C}$ in plastic
Date/Time Added to Test Chambers: $823 / 99$
Observations: $\quad$ silt,detritus
Collection Method: grab
Disposal

Source: BGCH5 Test Sediment
Collection Date $7 / 19 / 99 \quad$ Homogenized?: yes
Preservation: kept at $0-4^{\circ} \mathrm{C}$ in plastic
Date/Time Added to Test Chambers: 8/399
Observations:
Collection Method: grab
Shipment: overnight

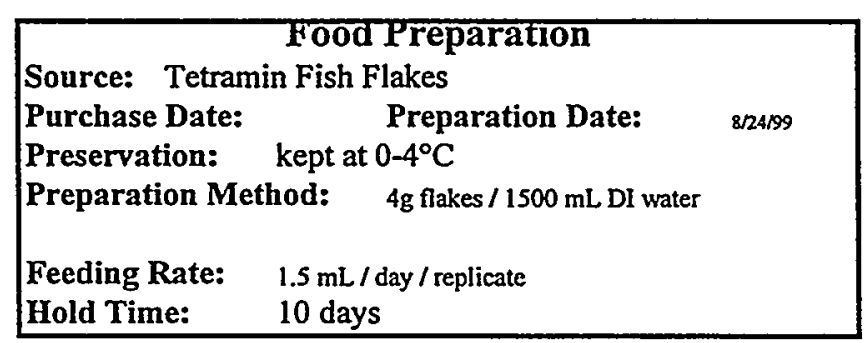

\begin{tabular}{|l|}
\hline \multicolumn{1}{c|}{ Test Chambers } \\
Type: Plastic $500 \mathrm{~mL}$ beakers \\
Sediment Volume: $\quad 100 \mathrm{~mL}$ \\
Sediment Depth $4 \mathrm{~cm}$ \\
$\begin{array}{l}\text { Overlying Water Volume: } \quad 175 \mathrm{~mL} \\
\text { Overlying Water Depth: } \quad 5 \mathrm{~cm} \\
\text { Aeration: } \quad \text { Not needed }\end{array}$ \\
\hline
\end{tabular}




\section{DAY CHIRONOMUS SEDIMENT TOXICITY TEST}

\begin{tabular}{|l|l|l|}
\hline Lab ID\# & Client: $\quad$ WSRC & Start Date: $8 / 24 / 99$ \\
\cline { 2 - 4 } T12308 & Sample Identification: BGCH5. & End Date: $9 / 3 / 99$ \\
\hline
\end{tabular}

\section{WATER CHEMISTRY TEST METHODS}

\begin{tabular}{|l|l|}
\hline $\begin{array}{l}\text { Temperature: SM 18th Ed. 2550 } \\
\text { Dissolved Oxygen: SM 18th Ed. 4500-O } \\
\text { pH: SM 18th Ed. 4500-H+ }\end{array}$ & $\begin{array}{l}\text { Alkalinity: SM 18th Ed.2320 } \\
\text { Hardness: SM 18th Ed. 2340 } \\
\text { Conductivity: SM 18th Ed. 2510 }\end{array}$ \\
\hline
\end{tabular}

\section{WATER CHEMISTRY TEST DATA - OVERLYING WATER}

CONTROL TREATMENT

\begin{tabular}{|c|c|c|c|c|c|c|}
\hline & Temp. & $\mathrm{pH}$ & D.o. & D.o. & Alkalinity & Ammonia \\
\hline Test Day & C & units & $\mathrm{mg} / \mathrm{L}$ & $\%$ sat. & Initial & Initial \\
\hline Start & 19.4 & 7.22 & 8.6 & $92 \%$ & 10.4 & 1.7 \\
\hline 1 & $\begin{array}{l}19.3 \\
\end{array}$ & & 7.2 & $77 \%$ & Final & Final \\
\hline 2 & 19.3 & & 6.9 & $74 \%$ & 14.0 & $<0.1$ \\
\hline 3 & 19.1 & & 8.1 & $87 \%$ & mgh & $\mathrm{mgh}$ \\
\hline 4 & 19.0 & & 6.8 & $73 \%$ & Cond. & Hardness \\
\hline 5 & 21.2 & & 7.4 & $83 \%$ & Initial & Initial \\
\hline 6 & 19.4 & & 6.0 & $65 \%$ & 58.7 & 9.5 \\
\hline 7 & 18.9 & & 6.2 & $65 \%$ & Final & Final \\
\hline 8 & 19.1 & & 6.3 & $68 \%$ & 175.0 & 9.5 \\
\hline 9 & 22.9 & & 5.9 & $68 \%$ & unhosicm & mgh \\
\hline 10 & 22.9 & 6.80 & 6.2 & $71 \%$ & |A & \\
\hline
\end{tabular}

\section{SURVIVAL AND GROWTH RESULTS}

CONTROL TREATMENT

\begin{tabular}{|c|c|c|c|c|c|c|}
\hline & \multicolumn{3}{|c|}{ \#Organisms } & \multirow[b]{2}{*}{$\begin{array}{c}\% \\
\text { Survival }\end{array}$} & \multirow{2}{*}{$\begin{array}{l}\text { Mean } \\
\text { Length } \\
(\mathrm{mm})\end{array}$} & \multirow{2}{*}{$\begin{array}{l}\text { Standard } \\
\text { Deviation }\end{array}$} \\
\hline & Initial & $\begin{array}{c}\text { Live at } \\
\text { End }\end{array}$ & $\begin{array}{l}\text { Dead } \\
\text { at End }\end{array}$ & & & \\
\hline$\overline{\mathrm{A}}$ & 10 & 2 & 8 & $20 \%$ & & \\
\hline$\overline{\mathrm{B}}$ & 10 & 8 & $\overline{2}$ & $80 \%$ & & \\
\hline C & 10 & 5 & 5 & $50 \%$ & & \\
\hline$\overline{\mathrm{D}}$ & 10 & 10 & 0 & $100 \%$ & & \\
\hline $\mathrm{E}$ & 10 & 7 & 3 & $70 \%$ & & \\
\hline $\mathbf{F}$ & 10 & 8 & 2 & $80 \%$ & & \\
\hline $\mathrm{G}$ & 10 & 7 & 3 & $70 \%$ & & \\
\hline $\mathrm{H}$ & 10 & spilled & 0 & $\overline{\mathrm{NA}}$ & & \\
\hline Mean & 10.0 & & & $67 \%$ & & \\
\hline
\end{tabular}

\begin{tabular}{|l|r|r|r|r|l|l|}
\hline \multicolumn{7}{|c|}{ SAMPLE TREATMENT } \\
\hline & \multicolumn{2}{|c|}{ \# Organisms } & Mean & Standard \\
& Initial & $\begin{array}{r}\text { Live at } \\
\text { End }\end{array}$ & $\begin{array}{c}\text { Dead } \\
\text { at End }\end{array}$ & $\begin{array}{c}\text { \% } \\
\text { Survival }\end{array}$ & $\begin{array}{l}\text { Length } \\
(\mathrm{mm})\end{array}$ & Deviation \\
\hline A & 10 & 0 & 10 & $0 \%$ & & \\
\hline B & 10 & 2 & 8 & $20 \%$ & & \\
\hline D & 10 & 2 & 8 & $20 \%$ & & \\
\hline E & 10 & 3 & 7 & $30 \%$ & & \\
\hline F & 10 & 1 & 9 & $10 \%$ & & \\
\hline G & 10 & 2 & 8 & $20 \%$ & & \\
\hline H & 10 & 2 & 8 & $20 \%$ & & \\
\hline Mean & 10 & 0 & 10 & $0 \%$ & & \\
\hline
\end{tabular}




\section{DAY CHIRONOMUS SEDIMENT TOXICITY TEST}

\begin{tabular}{|c|c|c|c|c|}
\hline \multirow{2}{*}{\begin{tabular}{|l} 
Lab ID\# \\
T12308
\end{tabular}} & \multicolumn{2}{|c|}{ Client: $\quad$ WSRC } & Start Date: & $8 / 24 / 99$ \\
\hline & Sample Identification: & BGCH5 & End Date: & 9/3/99 \\
\hline
\end{tabular}

\section{RESULTS}

\begin{tabular}{|l|r|}
\hline \multicolumn{2}{|c|}{$\begin{array}{c}\text { SURVIVAL DATA } \\
\text { Mean of Eight Replicates }\end{array}$} \\
\cline { 2 - 2 } & Mean \% \\
\hline Control & $67.1 \%$ \\
\hline Sample & $15.0 \%$ \\
\hline
\end{tabular}

\begin{tabular}{|l|l|}
\hline \multicolumn{2}{|c|}{\begin{tabular}{c} 
GROWTH DATA \\
Mean Length (mm) \\
\cline { 2 - 2 } \\
\cline { 2 - 2 }
\end{tabular}} \\
\hline Control & Mean \\
\hline Sample & NA \\
\hline
\end{tabular}

\section{STATISTICAL ANALYSES}

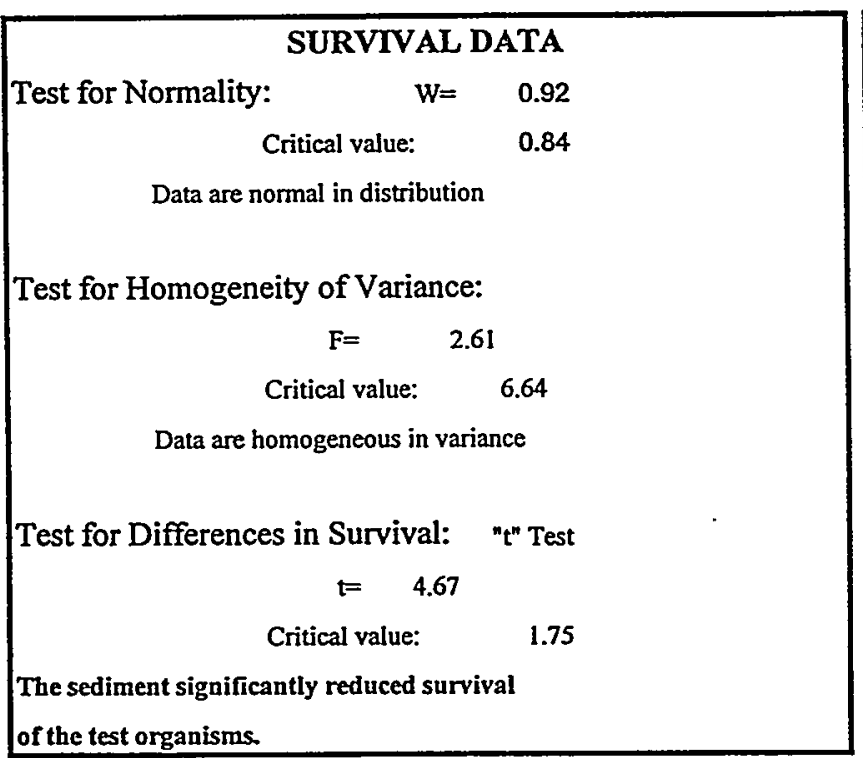

\begin{tabular}{|c|c|}
\hline \multicolumn{2}{|c|}{ GROWTH DATA } \\
\hline \multicolumn{2}{|l|}{ Test for Normality: } \\
\hline Critical value: & 0.84 \\
\hline \multicolumn{2}{|l|}{ NA } \\
\hline \multicolumn{2}{|c|}{ Test for Homogeneity of Variance: } \\
\hline$F=$ & NA \\
\hline Critical value: & 6.635 \\
\hline \multicolumn{2}{|l|}{ NA } \\
\hline \multicolumn{2}{|c|}{$\begin{array}{c}\text { Test for Differences in Survival: " } \mathrm{t} \text { " Test } \\
\qquad=\mathrm{NA}\end{array}$} \\
\hline Critical value: & 1.94 \\
\hline & $\approx$ \\
\hline
\end{tabular}

\section{COMMENTS}

Control survival was less than recommended minimum of $80 \%$; this is believed to be due to high coarse silt content of the sediment. Tubificid worms were also noted in several control replicates.

\begin{tabular}{|c|c|c|c|}
\hline $\begin{array}{l}\text { Principal Analyst: } \\
\text { Report Reviewed By: }\end{array}$ & $\begin{array}{l}\text { Marsha Leitzel } \\
\text { Robert W. Kelley, Ph.D. }\end{array}$ & 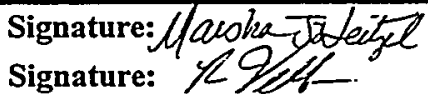 & $\begin{array}{l}\text { Date: } 10-19-79 \\
\text { Date: } 10-19-98\end{array}$ \\
\hline
\end{tabular}

\section{CONCLUSIONS}

The sediment reduces the survival of the test organisms. 


\title{
10 DAY CHIRONOMUS SEDIMENT TOXICITY TEST
}

\author{
EPA/600/R-94/024 \\ Test Organism: Chironomus tentans
}

\author{
Client: WSRC \\ Sample Identification: $\quad$ D-7
}

Test Start Date: $\quad 8 / 24 / 99$ 


\section{DAY CHIRONOMUS SEDIMENT TOXICITY TEST}

\begin{tabular}{|l|l|l|l|}
\hline Lab ID $\#$ & Client: & \multicolumn{1}{|c|}{ WSRC } & Start Date: \\
\hline T12304 & Sample Identification: $\quad$ D-7 & End Date: & $9 / 3 / 99$ \\
\hline
\end{tabular}

\section{Test System}

\section{EPA/600/R-94/024}

The test was set as a Pass/Fail test with a control treatment and one sample treatment. Each treatment replicate consisted of $100 \mathrm{~g}$ of sediment and $175 \mathrm{~mL}$ of dilution water. There were eight replicates for each treatment. Ten test organisms were placed in each replicate. Sediment from a local stream was used in the control treatment. Test organisms were fed with YCT food. The test was conducted at a temperature of $23^{\circ} \mathrm{C}$ and a light cycle of $16 \mathrm{hr}$ light $/ 8 \mathrm{hr}$ dark. Test vessels were $500 \mathrm{~mL}$ plastic beakers placed in a constant temperature incubator room.

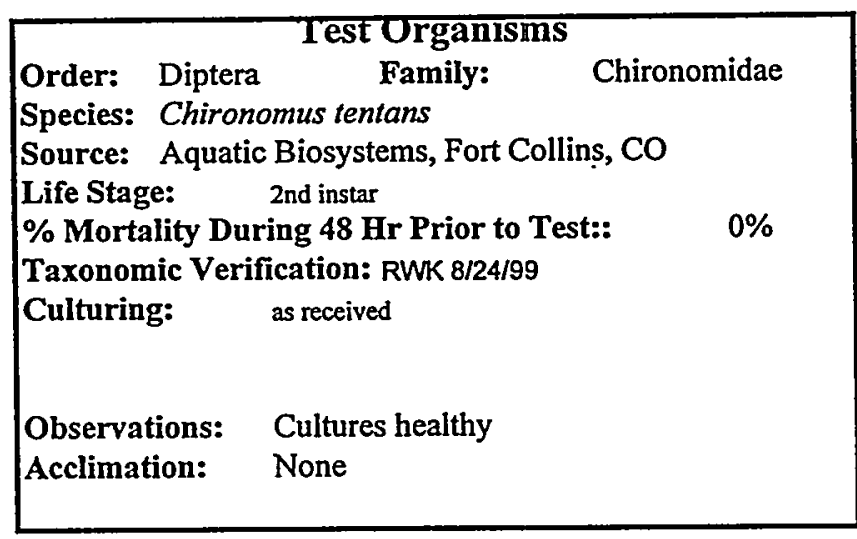

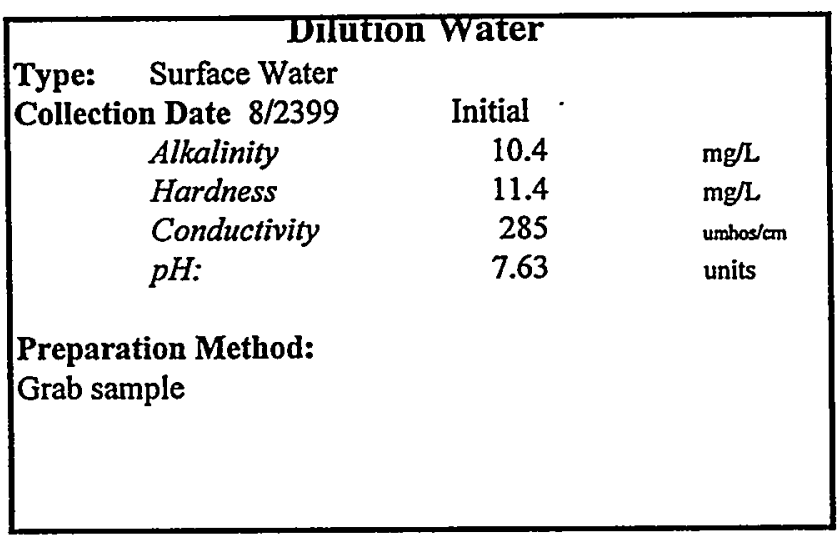

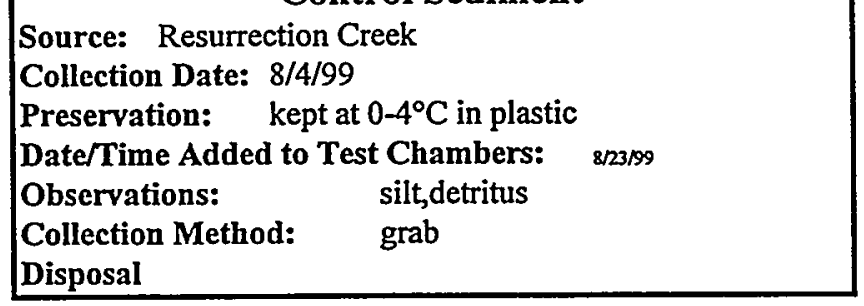

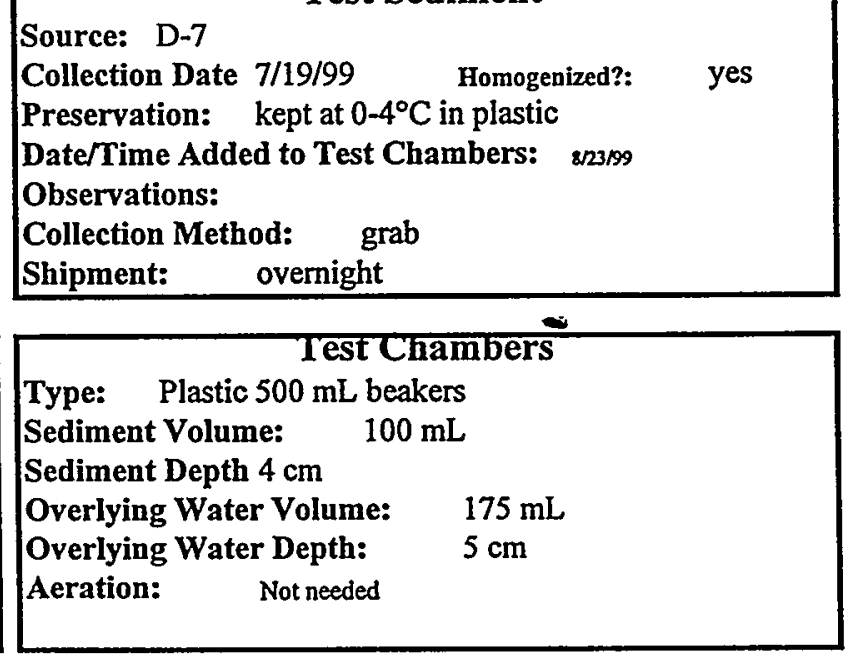

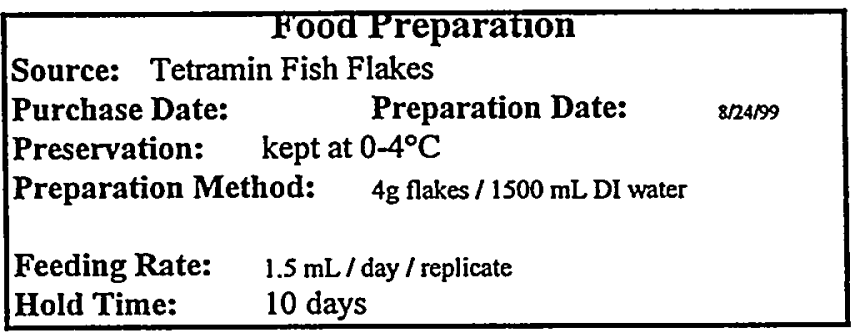




\section{DAY CHIRONOMUS SEDIMENT TOXICITY TEST}

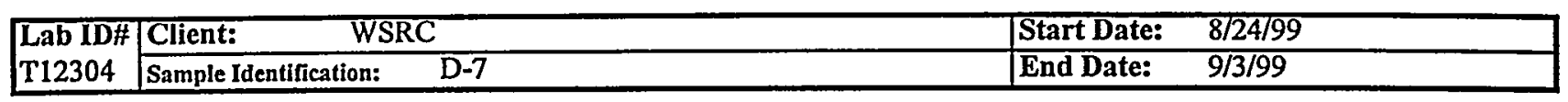

\section{WATER CHEMISTRY TEST METHODS}

\begin{tabular}{|l|l|}
\hline Temperature: SM 18th Ed. 2550 & Alkalinity: SM 18th Ed. 2320 \\
Dissolved Oxygen: SM 18th Ed. 4500-O & Hardness: SM 18th Ed. 2340 \\
pH: SM 18th Ed. 4500-H+ & Conductivity: SM 18th Ed. 2510 \\
\hline
\end{tabular}

\section{WATER CHEMISTRY TEST DATA - OVERLYING WATER}

CONTROL TREATMENT

\begin{tabular}{|c|c|c|c|c|c|c|}
\hline & Temp. & $\mathrm{pH}$ & D.O. & D.O. & Alkalinity & Ammonia \\
\hline Test Day & C & units & $\mathrm{mg} / \mathrm{L}$ & $\%$ sat. & Initial & Initial \\
\hline Start & 19.4 & 7.22 & 8.6 & $92 \%$ & 10.4 & 1.7 \\
\hline 1 & 19.3 & & 7.2 & $77 \%$ & Final & Final \\
\hline 2 & 19.3 & & 6.9 & $74 \%$ & 14.0 & $<0.1$ \\
\hline 3 & 19.1 & & 8.1 & $87 \%$ & mgl & $\mathrm{mgh}$ \\
\hline 4 & 19.0 & & 6.8 & $73 \%$ & Cond. & Hardness \\
\hline 5 & 21.2 & & 7.4 & $83 \%$ & Initial & Initial \\
\hline 6 & 19.4 & & 6.0 & $65 \%$ & 58.7 & 9.5 \\
\hline 7 & 18.9 & & 6.2 & $65 \%$ & Final & Finat \\
\hline 8 & 19.1 & & 6.3 & $68 \%$ & 175.0 & 9.5 \\
\hline 9 & 22.9 & & 5.9 & $68 \%$ & umhos/cm & $m g h$ \\
\hline 10 & 22.9 & 6.80 & 6.2 & $71 \%$ & Aeration: $\mathrm{n}$ & one \\
\hline
\end{tabular}

\section{SURVIVAL AND GROWTH RESULTS}

CONTROL TREATMENT

\begin{tabular}{|c|c|c|c|c|c|c|}
\hline & \multicolumn{3}{|c|}{ \# Organisms } & \multirow[b]{2}{*}{$\begin{array}{c}\% \\
\text { Survival } \\
\end{array}$} & \multirow{2}{*}{$\begin{array}{l}\text { Mean } \\
\text { Length } \\
\text { (mm) }\end{array}$} & \multirow{2}{*}{$\begin{array}{l}\text { Standard } \\
\text { Deviation }\end{array}$} \\
\hline & Initial & $\begin{array}{l}\text { Live at } \\
\text { End }\end{array}$ & $\begin{array}{l}\text { Dead } \\
\text { at End }\end{array}$ & & & \\
\hline $\mathrm{A}$ & 10 & 2 & 8 & $20 \%$ & & \\
\hline $\bar{B}$ & 10 & $\overline{8}$ & 2 & $80 \%$ & & \\
\hline $\bar{C}$ & 10 & 5 & 5 & $50 \%$ & & \\
\hline $\bar{D}$ & 10 & 10 & 0 & $100 \%$ & & \\
\hline $\mathrm{E}$ & 10 & 7 & 3 & $70 \%$ & & \\
\hline$\overline{\mathrm{F}}$ & 10 & $\overline{8}$ & 2 & $80 \%$ & & \\
\hline $\bar{G}$ & 10 & 7 & 3 & $70 \%$ & & \\
\hline$\overline{\mathrm{H}}$ & 10 & spilled & $\overline{0}$ & NA & & \\
\hline Mean & 10.0 & & & $67 \%$ & & \\
\hline
\end{tabular}

SAMPLE TREATMENT

\begin{tabular}{|c|c|c|c|c|c|c|}
\hline & Temp. & $\mathrm{pH}$ & D.O. & D.O. & Alkalinity & Ammonia \\
\hline Test Day & C & units & $\mathrm{mg} / \mathrm{L}$ & $\%$ sat. & Initial & Initial \\
\hline Start & 19.4 & 7.22 & 8.6 & $\overline{92 \%}$ & 10.4 & 1.7 \\
\hline 1 & 19.3 & & 8.0 & $86 \%$ & Final & Final \\
\hline 2 & 19.3 & & 7.4 & $80 \%$ & 10.9 & $<0.1$ \\
\hline 3 & 19.1 & & 7.3 & $78 \%$ & $\mathrm{mgl}$ & $\mathrm{mgh}$ \\
\hline 4 & 19.0 & & 7.6 & $82 \%$ & Cond. & Hardness \\
\hline 5 & 21.6 & & 7.0 & $79 \%$ & Initial & Initial \\
\hline 6 & 19.4 & & 7.3 & $78 \%$ & 58.7 & 9.5 \\
\hline 7 & 18.9 & & 7.6 & $80 \%$ & Final & Final \\
\hline 8 & 19.1 & & 7.6 & $82 \%$ & 182.0 & 5.70 \\
\hline 9 & 22.9 & & 7.1 & $82 \%$ & umhos/cm & $\mathrm{mgl}$ \\
\hline 10 & 22.9 & 7.17 & 7.2 & $83 \%$ & Aeration & \\
\hline
\end{tabular}

\begin{tabular}{|c|c|c|c|c|c|c|}
\hline & $\#$ & $\begin{array}{l}\text { rganisr } \\
\text { Live at } \\
\text { End }\end{array}$ & $\begin{array}{l}\text { DS } \\
\text { Dead } \\
\text { at End }\end{array}$ & $\begin{array}{c}\% \\
\text { Survival }\end{array}$ & $\begin{array}{l}\text { Mean } \\
\text { Length } \\
\text { (mm) }\end{array}$ & $\begin{array}{l}\text { Standard } \\
\text { Deviation }\end{array}$ \\
\hline $\bar{A}$ & 10 & 4 & 6 & $40 \%$ & & \\
\hline $\mathrm{B}$ & 10 & 5 & 5 & $50 \%$ & & \\
\hline $\bar{C}$ & 10 & 6 & 4 & $60 \%$ & & \\
\hline $\mathrm{D}$ & 10 & 3 & 7 & $30 \%$ & & \\
\hline $\bar{E}$ & 10 & 9 & 1 & $90 \%$ & & \\
\hline $\bar{F}$ & 10 & 7 & $\overline{3}$ & $70 \%$ & & \\
\hline $\mathbf{G}$ & 10 & 1 & 9 & $10 \%$ & & \\
\hline $\mathrm{H}$ & 10 & 5 & 5 & $50 \%$ & & \\
\hline Mean & 10.0 & & & $50 \%$ & & \\
\hline
\end{tabular}




\section{DAY CHIRONOMUS SEDIMENT TOXICITY TEST}

\begin{tabular}{|c|c|c|c|}
\hline Lab ID\# & Client: & Start Date: & $8 / 24 / 99$ \\
\hline T12304 & Sample Identification: $\quad$ D-7 & End Date: & $9 / 3 / 99$ \\
\hline
\end{tabular}

\section{RESULTS}

\begin{tabular}{|l|r|}
\hline \multicolumn{2}{|c|}{$\begin{array}{c}\text { SURVIVAL DATA } \\
\text { Mean of Eight Replicates }\end{array}$} \\
\cline { 2 - 2 } & Mean \% \\
\hline Control & $67.1 \%$ \\
\hline Sample & $50.0 \%$ \\
\hline
\end{tabular}

\begin{tabular}{|l|l|}
\hline \multicolumn{2}{|c|}{$\begin{array}{c}\text { GROWTH DATA } \\
\text { Mean Length (mm) }\end{array}$} \\
\cline { 2 - 2 } & \multicolumn{1}{|c|}{ Mean } \\
\hline Control & NA \\
\hline Sample & NA \\
\hline
\end{tabular}

\section{STATISTICAL ANALYSES}

\begin{tabular}{|crr}
\hline & \multicolumn{3}{c}{ SURVTVAL DATA } \\
Test for Normality: $\quad \mathrm{W}=$ & 0.94 \\
& Critical value: & 0.84
\end{tabular}

Data are nomal in distribution

Test for Homogeneity of Variance:

$$
\begin{array}{cr}
F= & 1.36 \\
\text { Critical value: } & 6.64
\end{array}
$$

Data are homogeneous in variance

Test for Differences in Survival: " $\mathrm{t}^{n}$ Test

$$
\begin{gathered}
t=1.35 \\
\text { Critical value: } \quad 1.75
\end{gathered}
$$

The sediment did not significantly reduce survival

of the test organisms.

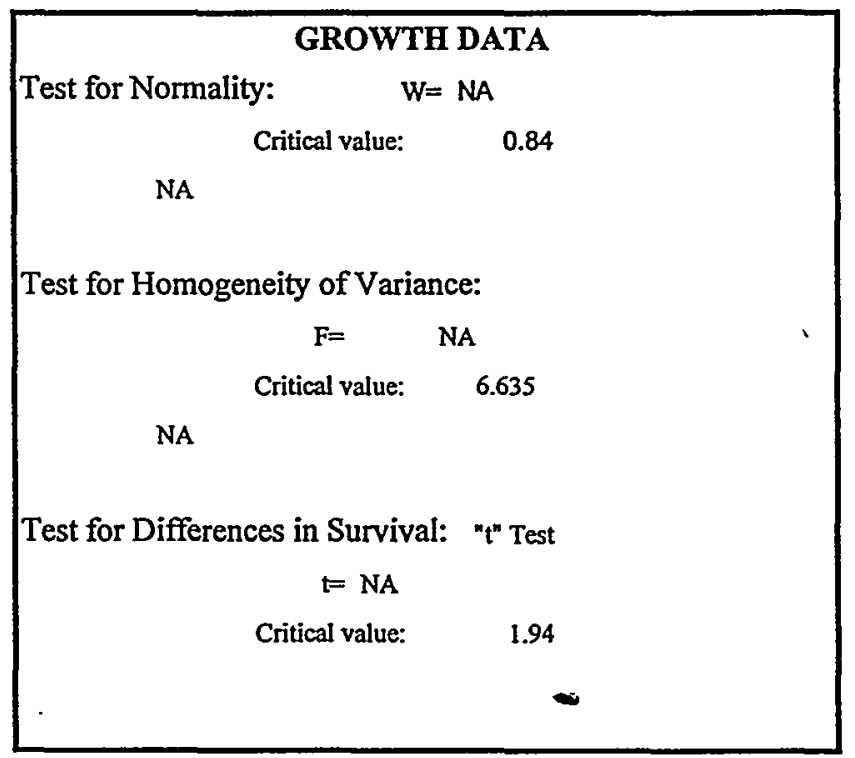

\section{COMMENTS}

Control survival was less than recommended minimum of $80 \%$; this is believed to be due to high coarse silt content of the sediment. Tubificid worms were also noted in several control replicates.

\begin{tabular}{|lll|}
\hline Principal Analyst: & Marsha Leitzel & Signature: \\
Report Reviewed By: & Robert W. Kelley, Ph.D. & Signature Masha
\end{tabular}

\section{CONCLUSIONS}

The sediment does not reduce the survival of the test organisms. 